\title{
Patient Bed Transfer Analysis Using Pressure Sensor Arrays
}

By

Idana Veledar, B.Eng.

A thesis submitted to

The Faculty of Graduate Studies and Research

in partial fulfilment of

the degree requirements of

Master of Applied Science

Ottawa-Carleton Institute for

Biomedical Engineering

Department of Systems and Computer Engineering

Carleton University

Ottawa, Ontario, Canada

August 2009

Copyright (C)

2009 - Idana Veledar 


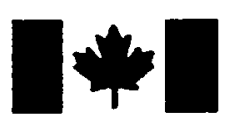

\author{
Library and Archives \\ Canada \\ Published Heritage \\ Branch \\ 395 Wellington Street \\ Ottawa ON K1A ON4 \\ Canada
}

Bibliothèque et

Archives Canada

Direction du

Patrimoine de l'édition

395, rue Wellington

Ottawa ON K1A ON4

Canada
Your file Votre référence
ISBN: $978-0-494-60237-9$
Our file Notre référence
ISBN: $978-0-494-60237-9$
NOTICE:

The author has granted a nonexclusive license allowing Library and Archives Canada to reproduce, publish, archive, preserve, conserve, communicate to the public by telecommunication or on the Internet, loan, distribute and sell theses worldwide, for commercial or noncommercial purposes, in microform, paper, electronic and/or any other formats.

The author retains copyright ownership and moral rights in this thesis. Neither the thesis nor substantial extracts from it may be printed or otherwise reproduced without the author's permission.

\begin{abstract}
AVIS:
L'auteur a accordé une licence non exclusive permettant à la Bibliothèque et Archives Canada de reproduire, publier, archiver, sauvegarder, conserver, transmettre au public par télécommunication ou par l'Internet, prêter, distribuer et vendre des thèses partout dans le monde, à des fins commerciales ou autres, sur support microforme, papier, électronique et/ou autres formats.
\end{abstract}

L'auteur conserve la propriété du droit d'auteur et des droits moraux qui protège cette thèse. $\mathrm{Ni}$ la thèse ni des extraits substantiels de celle-ci ne doivent être imprimés ou autrement reproduits sans son autorisation.
In compliance with the Canadian Privacy Act some supporting forms may have been removed from this thesis.

While these forms may be included in the document page count, their removal does not represent any loss of content from the thesis.
Conformément à la loi canadienne sur la protection de la vie privée, quelques formulaires secondaires ont été enlevés de cette thèse.

Bien que ces formulaires aient inclus dans la pagination, il n'y aura aucun contenu manquant.

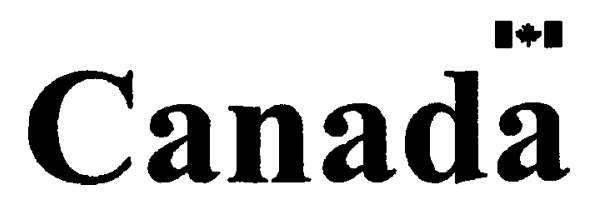




\section{Abstract}

Monitoring and analyzing sit-to-stand movements performed by an older person when rising from their bed could be used as a measure of health and mobility. Pressure patterns for four adult test groups were collected using a pressure sensitive mat technology and analyzed using image processing and signal processing techniques.

Pressure images were generated from the raw sensor data and analyzed using four region of interest algorithms. The hip and hand regions were successfully extracted and the best results were obtained using the binary cluster detection algorithm. The sensor data was summed to create a sit-to-stand time pressure signal and an automated algorithm was proposed to segment the signal into three phases. The results extracted individual phase times as well as total sit-to-stand times. The algorithm performance was verified using video analysis data.

The timing results were used to evaluate the health and mobility of different participant groups and could be used to monitor changes in sit-to-stand movements performed by occupants living in smart homes. 


\section{Acknowledgements}

I would like to express my gratitude to my co-supervisors, Dr. Rafik Goubran and Dr. Frank Knoefel, for their expertise, guidance and wisdom. I appreciate the time and effort that was provided by both supervisors throughout my graduate experience.

I would like to thank the Natural Sciences and Engineering Research Council of Canada (NSERC) for their financial assistance as well as the Canadian Institutes of Health Research (CIHR), Ontario Graduate Scholarship in Science and Technology (OGSST), the Department of Systems and Computer Engineering and Carleton University.

I must also acknowledge Jenna Wiens, Nick Foubert, Pam Carlson and Jodie Taylor for their efforts and assistance. A very special thanks goes to Amaya Arcelus for her technical advice, motivation and editing assistance. Finally I would like to thank my family for the support and encouragement they have provided me throughout my entire life. 


\section{Table of Contents}

$\begin{array}{lll}\text { Abstract } & \text { iii }\end{array}$

Acknowledgements

Table of Contents $\quad v$

$\begin{array}{ll}\text { List of Tables } & \text { ix }\end{array}$

List of Figures $x$

$\begin{array}{ll}\text { Chapter 1: Introduction } & 1\end{array}$

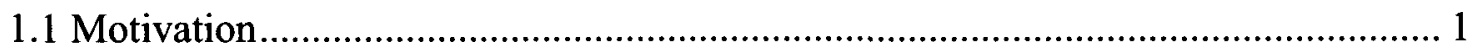

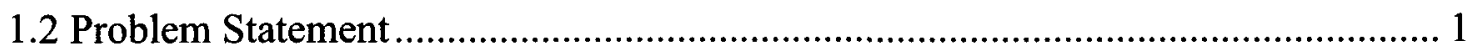

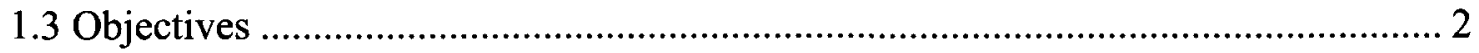

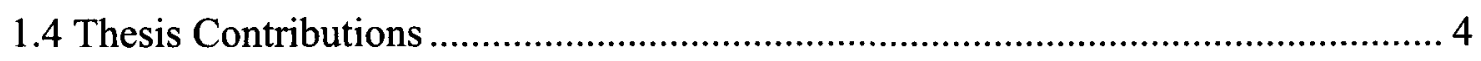

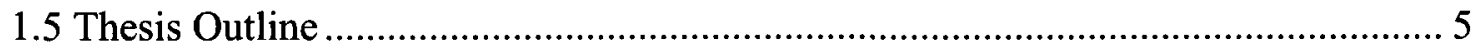

$\begin{array}{ll}\text { Chapter 2: Background Review } & 7\end{array}$

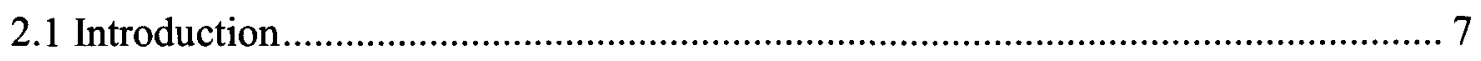

2.2 Smart Home Technologies................................................................................. 7

2.3 Human Activity Monitoring ……………........................................................ 9

2.4 Sit-to-stand Analysis ......................................................................................... 9

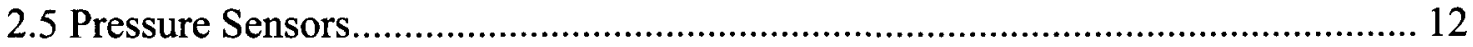

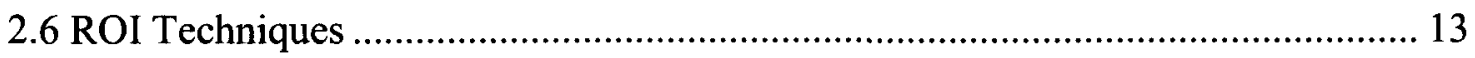

$\begin{array}{ll}\text { Chapter 3: Experiment Setup } & 16\end{array}$

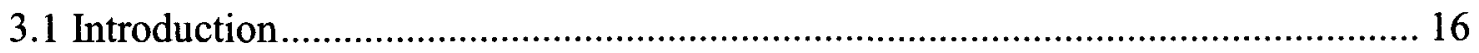

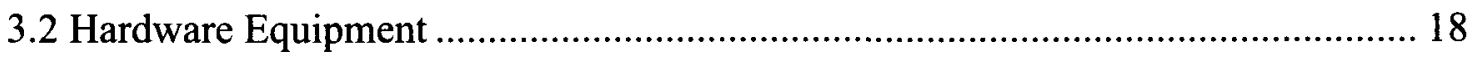

3.2.1 Pressure Sensitive Bed Mats ....................................................................... 18

3.2.2 Pressure Sensitive Floor Mat .......................................................................... 19 


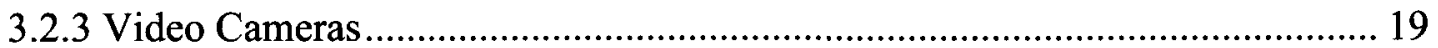

3.2.4 Equipment Setup ..................................................................................... 19

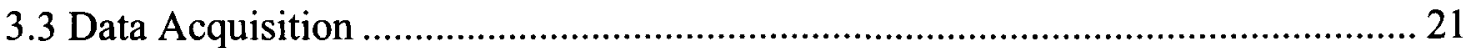

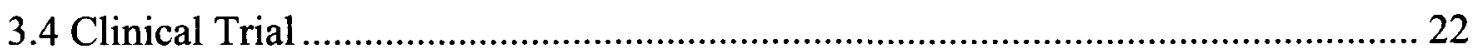

Chapter 4: Generation of Pressure Images $\quad 24$

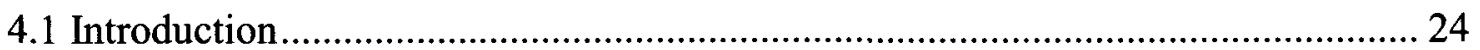

4.2 Mapping Pressure Sensor Array Data................................................................. 26

4.3 Spatial Interpolation of Sensor Array Data............................................................ 27

4.3.1 Interpolation Using Gaussian Kernel Convolution........................................... 27

4.3.2 Gaussian Kernel Convolution and Polynomial Interpolation .......................... 28

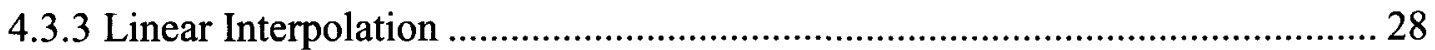

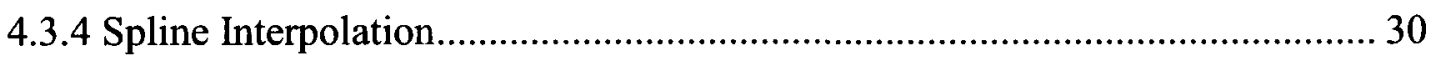

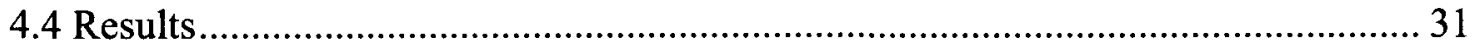

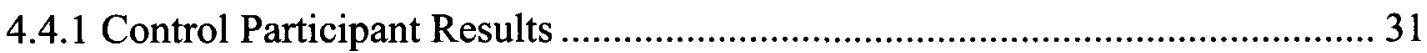

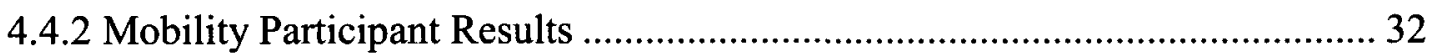

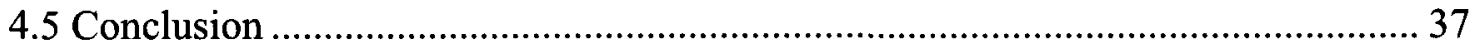

$\begin{array}{lr}\text { Chapter 5: ROI Extraction in Spatial Domain } & 38\end{array}$

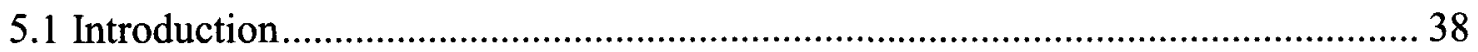

5.2 Ideal Sit-to-stand Transfer ................................................................................ 39

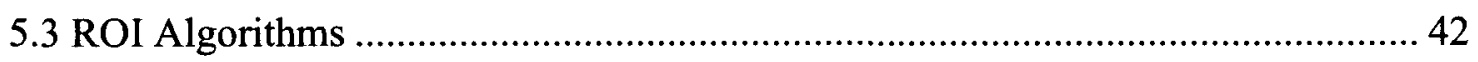

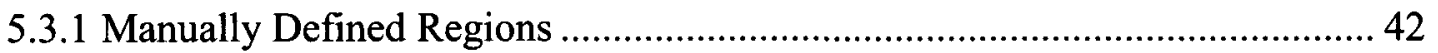

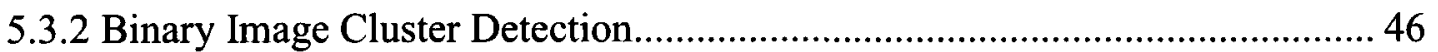

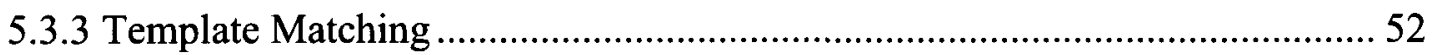

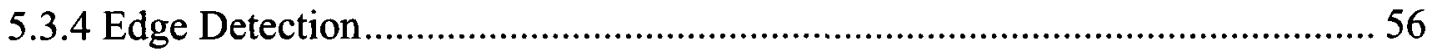

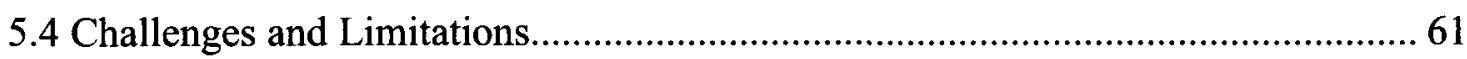

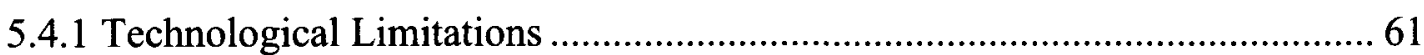

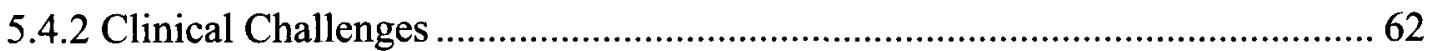

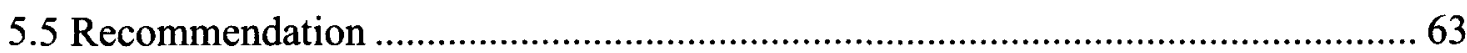

Chapter 6: Sit-to-stand Segmentation and Timing 66 


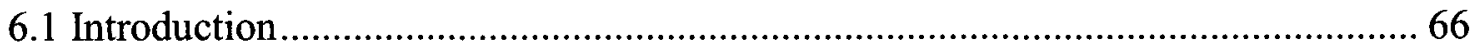

6.2 Sit-to-stand Phase Detection Algorithm …………….......................................... 71

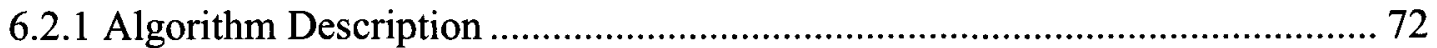

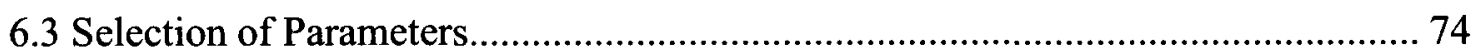

6.3.1 Weight Shift Parameter Discussion ................................................................ 74

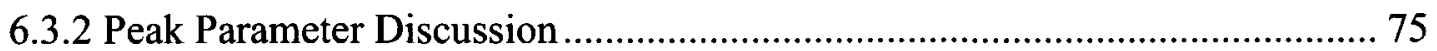

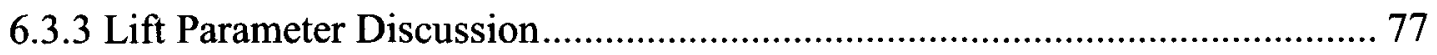

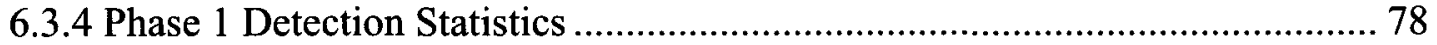

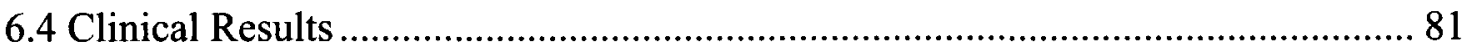

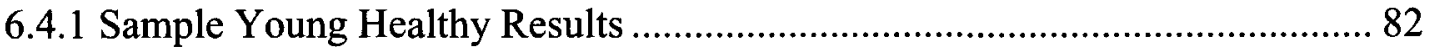

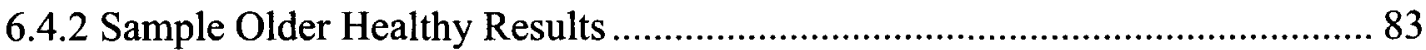

6.4.3 Sample Older Post-Stroke Results ............................................................... 84

6.4.4 Sample Older Post-Hip Results ..................................................................... 85

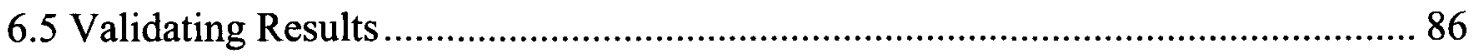

6.6 Bounce Detection for Functionally Impaired Participants........................................ 89

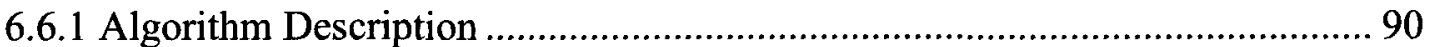

6.6.2 Functionally Impaired Participant Results................................................... 92

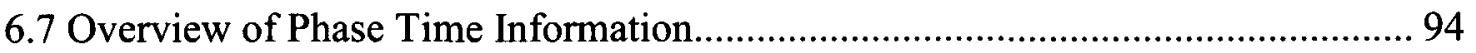

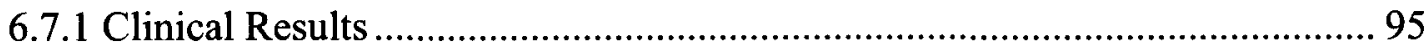

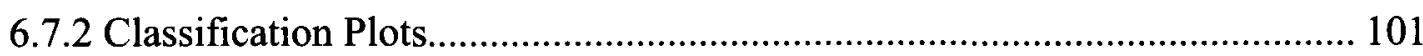

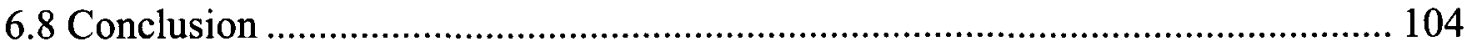

$\begin{array}{ll}\text { Chapter 7: Conclusion } & 106\end{array}$

7.1 Summary of Results and Contributions .............................................................. 106

7.2 Recommendations for Future Work................................................................. 107

$\begin{array}{ll}\text { List of References: } & 108\end{array}$

Appendix A: Equipment Setup User Manual 115

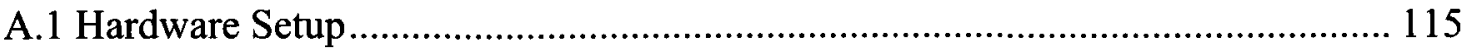

A.1.1 Three Bed Mat Configuration ................................................................ 115

A.1.2 Individual Bed Mat to Computer Connection................................................. 115 
A.1.3 Floor Mat Configuration ........................................................................ 116

A.1.4 Floor Mat to Computer Connection .............................................................. 116

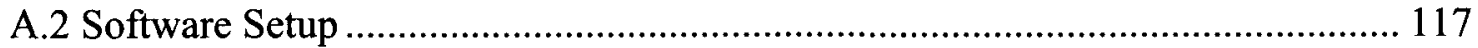

A.1.5 Computer Setup.......................................................................................... 117

A.1.6 NPort (black box) Setup............................................................................ 118

Appendix B: Data Acquisition User Manual 119

A.3 Initializing Connection to Bed Mats ................................................................. 119

A.4 Floor Mat and Bed Mat Connections ................................................................. 121

A.5 Data Acquisition ................................................................................................ 122

A.6 Troubleshooting ……………………............................................................ 125 


\section{List of Tables}

Table 6.1: Young healthy phase 1 detection success rate ......................................... 80

Table 6.2: Young healthy participant average sit-to-stand time using test parameters .... 81 


\section{List of Figures}

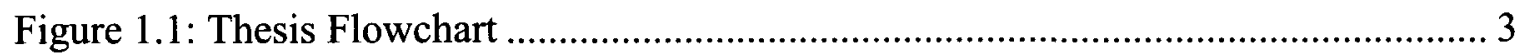

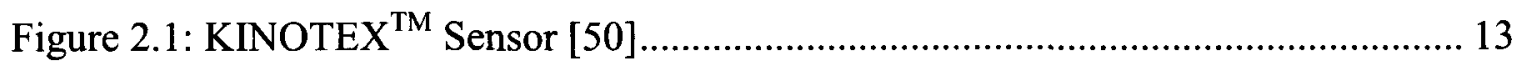

Figure 3.1: Thesis Flowchart ................................................................................... 17

Figure 3.2: Experiment Setup Flowchart ................................................................... 17

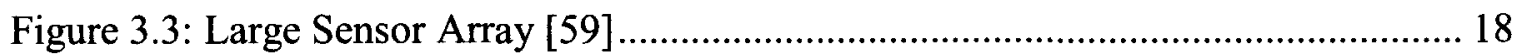

Figure 3.4: High Density Floor Sensor [59] ………............................................... 19

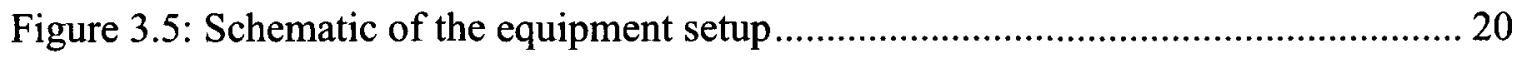

Figure 3.6: Bed mat installation at the Élisabeth Bruyère hospital................................... 20

Figure 3.7: Equipment setup installed in the Élisabeth Bruyère hospital ........................ 21

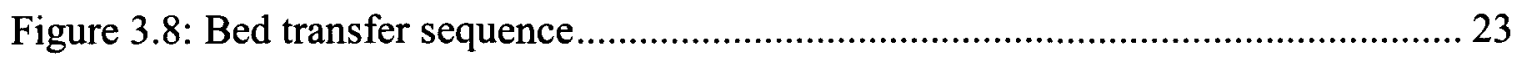

Figure 4.1: Thesis Flowchart .................................................................................... 24

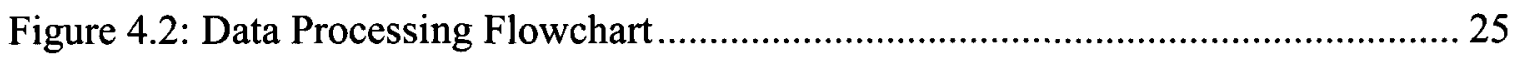

Figure 4.3: Pressure sensor (taxel) locations ........................................................... 26

Figure 4.4: Pressure image created using 2D convolution with a Gaussian kernel.......... 28

Figure 4.5: Taxel mapping used to create linear interpolation pressure images............... 29

Figure 4.6: Comparison of four techniques used to create pressure images representing

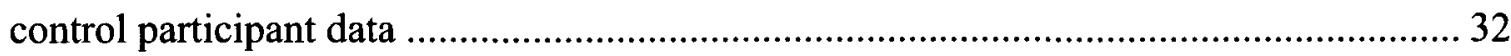

Figure 4.7: Comparison of four techniques used to create pressure images representing a seated participant who is pushing on the bed with their right hand.................................. 34 Figure 4.8: Comparison of four techniques used to create pressure images representing a seated participant who is pushing on the bed with their left hand ..................................... 35

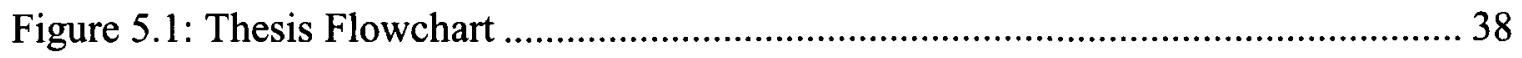

Figure 5.2: Region of Interest Extraction in Spatial Domain Flowchart .......................... 39

Figure 5.3: Sit-to-stand sequence.................................................................................... 41

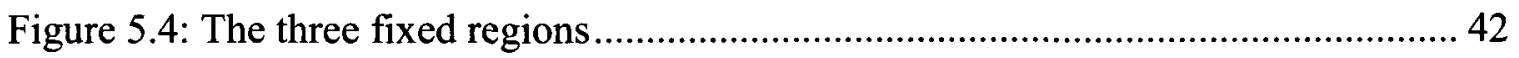


Figure 5.5: Ideal sit-to-stand transfer collected from control participant ......................... 43

Figure 5.6: Sit-to-stand transfer using the left hand only (control participant) ................ 44

Figure 5.7: Sit-to-stand transfer using the right hand only (control participant) .............. 45

Figure 5.8: Sample greyscale to binary image conversion ............................................... 46

Figure 5.9: Perimeter results for four different threshold values..................................... 47

Figure 5.10: Region detection results for a three image sequence .................................. 49

Figure 5.11: Perimeter results for a three image sequence .............................................. 50

Figure 5.12: Labelled detected regions results for a three image sequence...................... 51

Figure 5.13: Detected region when hands are placed too close to the hips ...................... 52

Figure 5.14: Detected region when subject is only using their left hand and it is placed

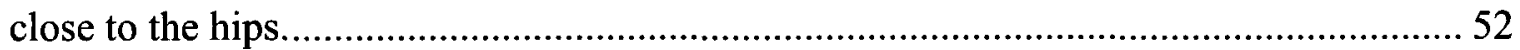

Figure 5.15: Template matching using rectangle mask image ………………............... 53

Figure 5.16: Template matching using sample hip mask image....................................... 54

Figure 5.17: Template matching using larger sample hip mask image ............................ 54

Figure 5.18: Template matching using largest sample hip mask image ........................... 55

Figure 5.19: Template matching using square mask image.............................................. 56

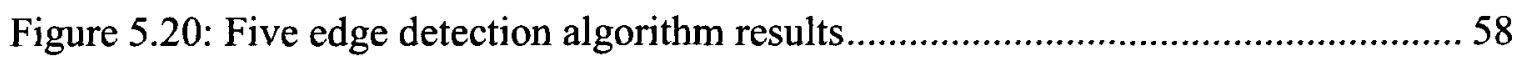

Figure 5.21: Three edge detection algorithm results ....................................................... 59

Figure 5.22: Canny edge detection result .................................................................... 59

Figure 5.23: Example displaying the main disadvantage of edge detection...................... 60

Figure 5.24: A sequence of two images extracted from a sit-to-stand sequence .............. 64

Figure 5.25: Decreasing pressure and increasing pressure regions ..................................... 64

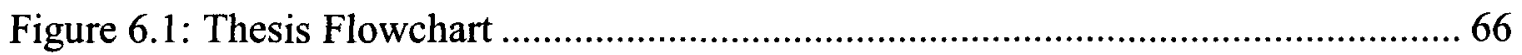

Figure 6.2: Sit-to-stand Segmentation and Timing Flowchart.......................................... 67

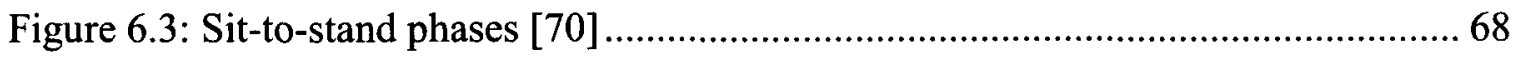

Figure 6.4: Simulated pressure curve showing an ideal sit-to-stand movement................69

Figure 6.5: Total pressure values for three participants with their corresponding weight

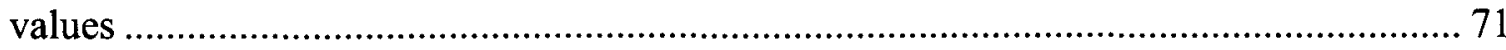

Figure 6.6: Sit-to-stand transfer \#1 for older healthy participant \#3649 .......................... 73

Figure 6.7: Sample plot comparing two different weight shift parameters ...................... 75

Figure 6.8: Sample plot comparing two different peak parameters................................... 76 
Figure 6.9: Sample plot comparing two different lift parameters

Figure 6.10: Sample plot comparing two different weight shift and peak parameters ..... 78

Figure 6.11: Sit-to-stand transfer \#12 for young healthy participant \#2164 ...................... 82

Figure 6.12: Sit-to-stand transfer \#23 for older healthy participant \#5265 ...................... 83

Figure 6.13: Sit-to-stand transfer \#2 for post-stroke participant \#6996............................. 84

Figure 6.14: Sit-to-stand transfer \#8 for post-hip participant \#8144 ............................... 85

Figure 6.15: Comparing participant results to validate algorithm ................................... 87

Figure 6.16: Comparing average sit-to-stand times for each participant ............................ 87

Figure 6.17: Comparing sit-to-stand times for individual transfers.................................. 88

Figure 6.18: Sit-to-stand transfer \#1 for post-stroke participant \#1269........................... 89

Figure 6.19: Sample plots for functionally impaired participant movements .................. 91

Figure 6.20: Sit-to-stand transfer \#1 for post-stroke participant \#1269.............................. 92

Figure 6.21: Sit-to-stand transfer \#8 for post-stroke participant \#1269............................ 93

Figure 6.22: Post-stroke participant \# 1269 movement summary ...................................... 94

Figure 6.23: Phase 1 transfer times and average times for 5 young healthy participants. 95

Figure 6.24: Phase 1 times for all four participant groups................................................. 96

Figure 6.25: Phase 2 times for all four participant groups.............................................. 97

Figure 6.26: Phase 3 times for all four participant groups.............................................. 99

Figure 6.27: Total sit-to-stand times for all four participant groups................................ 100

Figure 6.28: Phase 1 vs. Phase 2 classification plot for all four participant groups ....... 101

Figure 6.29: Phase 1 vs. Phase 2 classification plot for healthy and functionally impaired groups

Figure 6.30: Phase 1 vs. Phase 3 classification plot for all four participant groups ....... 103 Figure 6.31: Phase 1 vs. Phase 3 classification plot for healthy and functionally impaired groups 104 


\section{Chapter 1:}

\section{Introduction}

\subsection{Motivation}

There is a great demand for research in the field of patient monitoring, particularly monitoring in the home. The research motivation is driven by the possibility of having smart homes provide future benefits to seniors as well as to nurses and caregivers at hospitals and old age homes. This type of research aims to provide seniors with unobtrusive monitoring in their homes therefore allowing them to live independently longer. Embedding equipment such as pressure sensors into furniture will allow for accurate data collection from the occupant because all of the necessary movements will be performed in their home rather than in a clinical environment.

Monitoring movements such as the sit-to-stand transfer from a bed is important because, although seemingly a very simple task elderly people find it quite difficult to perform and therefore it can be used as a measure of health.

\subsection{Problem Statement}

The thesis research focuses on analyzing patient bed transfers using a pressure sensitive technology. More specifically the sit-to-stand movement will be evaluated using data collected from sensors located underneath the bed mattress. Analyzing the sit-to-stand 
movement is important in assessing the mobility of older healthy subjects as well as assessing the rehabilitation progress of older recovering subjects.

The Mobility project involved collecting data from four different participant groups: young and older healthy subjects, and older patients recuperating from a stroke or hip fracture. Designing and implementing methods for analyzing the sit-to-stand data and differentiating between the pressure patterns produced by the four adult test groups. Monitoring the sit-to-stand movements of a post-stroke participant over time, would hopefully result in their mobility level progressing to the same level as an older healthy participant.

\subsection{Objectives}

The thesis goal is to investigate and analyze sit-to-stand movements performed by young, older and functionally impaired participants. The algorithms used to assess patient mobility will be installed in the smart home to monitor the occupants' movements and discover any deviations from the normal.

The team involved with this project is a multidisciplinary team involving both engineering experts and clinical experts. Data analysis will be performed by the engineering team to extract useful sit-to-stand parameters as suggested by the clinical team. The collaboration between clinical and engineering researchers working together on the Mobility project will result in both innovative analysis and results. 
A block diagram describing the thesis research process is shown below.

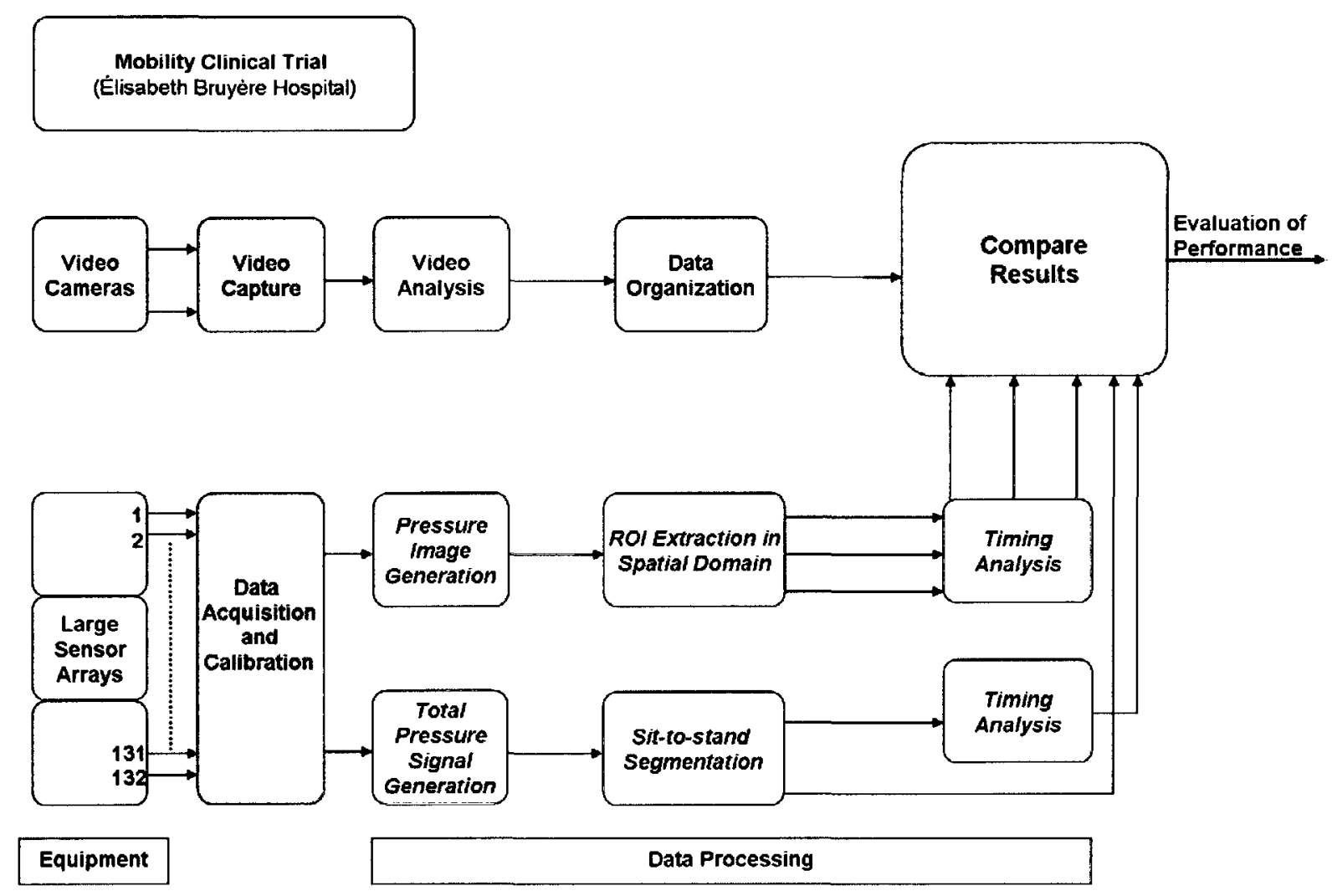

Figure 1.1: Thesis Flowchart

Raw pressure data will be processed to create pressure images, representing the sit-tostand movement as a set of frames in a movie sequence.

Region of interest algorithms will be implemented to extract information about the sit-to-stand movement one frame at a time. The use of hands will be identified as well as the timing information related to the hip and hand regions. The results will be verified by comparing them with results produced by clinicians.

The raw pressure data will be processed to create a pressure signal and the signal will be segmented into sit-to-stand phases. Timing information for each individual transfer will be extracted and a comparison will be performed between participants within a specific group as well as between participant groups as a whole. 
Timing data between the healthy and recovering group will be compared and a detailed analysis of the functionally impaired group will be conducted. This will include looking at unstable sit-to-stand transfers where the participant performs additional movements such as shuffling or bouncing their weight in order to generate enough momentum to rise from the bed.

\subsection{Thesis Contributions}

The following is a list of the research contributions that were made during this thesis research; each contribution will be explained thoroughly in the following chapters.

- Designed an experiment to acquire data from a pressure sensitive mat and supervised data collection. The data collection process consisted of running a clinical trial involving 25 recruited participants at the Élisabeth Bruyère Hospital.

- Designed and implemented several algorithms to process the raw pressure data to create a sequence of pressure images. Created time pressure signals from the individual sit-to-stand sequences.

- Proposed algorithms to analyze the pressure images by performing region of interest extraction in the spatial domain. Individual frames were processed to extract the regions of interest which included the hips and hands.

- Designed and implemented an automated algorithm to segment the sit-to-stand pressure signal into phases and performed time analysis on each phase.

- Designed and implemented an algorithm to detect additional movements performed by functionally impaired participants, during their sit-to-stand sequences. 


\subsection{Thesis Outline}

A block diagram describing the thesis flow was presented in Figure 1.1 in Section 1.3.

Chapter 2 will provide a literature review of smart homes, patient activity monitoring and sit-to-stand experiments which have been performed in the past. It will also introduce the pressure sensor technology and give an overview of region of interest algorithms used in later chapters.

Chapter 3 discusses the experiment setup including a description of the equipment that was used, the data acquisition process, and the clinical trial that was conducted at the Élisabeth Bruyère Hospital.

Chapter 4 is the first data processing chapter and it deals with creating pressure images using the raw pressure sensor data. Four different techniques are compared and results for each method are shown. The results are discussed and the most optimal technique is identified.

Chapter 5 performs region of interest extraction in the spatial domain using the pressure images created by Chapter 4 . The sit-to-stand transfer is made up of a sequence of frames and each is analyzed as a stand alone pressure image. Hip and hand regions are extracted using four different methods and timing analysis is performed on each region. Limitations for each technique are discussed and the best method is identified.

Chapter 6 sums the raw pressure data producing a pressure signal. The signal is segmented into three phases, weight shift, transition and lift. Together the three phases make up the sit-to-stand transfer. Timing information regarding each phase is extracted for individual participants as well as entire participant groups. A bounce detection algorithm is implemented in order to extract additional movements performed by the 
functionally impaired participants. The movements include shuffling, bouncing and unsuccessful transfers.

Chapter 7 presents the thesis conclusion which includes a summary of the results and contributions and a list of recommendations for future work.

References and appendices containing the equipment setup manual and data acquisition manual are listed at the end. 


\section{Chapter 2:}

\section{Background Review}

\subsection{Introduction}

This chapter presents a literature review on the topics of smart homes, human activity monitoring, sit-to-stand analysis, pressure sensors and an overview of several image processing techniques.

\subsection{Smart Home Technologies}

Canada along with the rest of the world has an aging population that is growing significantly faster than any other age group. Statistics show that in 2001 , only one in eight Canadians was over the age of 65 while projections show that in 2026 one in five Canadians will reach the age of 65 [1]. The number of medical services required by a person increases significantly due to health problems resulting from aging. Hospitals and nursing care facilities across the country are struggling to provide quality services due to cuts in funding and heavier workloads. Patients requiring light care are being replaced with patients who have much heavier medical needs, but the funding being provided for these services is less rather than more [2], [3].

There is therefore a growing demand for technology to help monitor and care for the elderly, in hospitals, nursing homes as well as their own homes. The development of 
smart home technologies is one suggested solution to this problem as it could monitor the health conditions of seniors while they are at home. Carleton University is involved a smart home project called Technology Assisted Friendly Environment for the Third Age (TAFETA) which is located at the Élisabeth Bruyère Hospital in Ottawa, Canada, which aims to provide researchers with a test facility. Arcelus et al. describes the prototype apartment which is currently equipped with a variety of sensor technologies [4]. Many studies have already been conducted in the field of smart home research. These include studies to create assessment tools to evaluate the independence of elders [5], [6], [7]; monitoring devices to evaluate patient activity [8] and detect distress situations in a smart home [9]. Many researchers are identifying new trends in the smart homes field [10], and discussing potential future technologies that might be useful [11].

Bartolomeu et al. describe several challenges in developing smart homes, such as having to design them to suit all types of people, security and privacy challenges as well as challenges in ensuring the patient was being monitored by a reliable and safe system [12]. Falls are the main cause of injuries amongst older people. This is the motivation for monitoring patients within their homes because it has been estimated that the resulting care costs in Canada are approximately $\$ 1$ billion annually [1].

Several different technologies have been proposed for patient tracking within a smart home. Saint-Bauzel et al. suggests that the number of sensors used to monitor patients should be small to ensure a natural living environment [13]. Technologies such a pressure sensors could be used to monitor patients throughout the home [13] as well as wireless bed sensors to monitor patients while they are sleeping [15]. 


\subsection{Human Activity Monitoring}

The research field of human activity monitoring is growing rapidly with research interests including detecting the daily activities of young active people [16], and monitoring the health of the elderly healthy [17] and unhealthy population [18].

Ermes et al. are attempting to detect daily activities including lying down, sitting, standing, walking and running performed by healthy people using 3D accelerometers as well as GPS [16]. Barralon et al. have published a study which uses three accelerometers to monitor patients and detect how much time they spent in each postural state (ie. lie, sit and walk) [19]. Knight et al. are trying to create an alarm which monitors an elderly patient and detects that they are trying to stand up from their wheelchair [20]. Najafi et al. are looking at elderly healthy patients [17] and Salarian et al. are looking at Parkinson's disease patients [18] while they perform daily physical movements, to help in patient movement monitoring and analysis.

One emerging technology that is being used for human activity monitoring is the use of pressure sensors. Studies have been conducted to analyze sitting postures [21] and hipprints [22] using pressure sensors placed on a seat; differentiating between objects and people by classifying movements as gross or slight using pressure sensors placed on a bed [23], and investigating stability in standing and walking using pressure sensors placed under the sole of the foot [24].

\subsection{Sit-to-stand Analysis}

The sit-to-stand movement is a simple task performed easily by most people on a daily basis. Older and unhealthy adults often find this simple movement very difficult to perform; therefore the sit-to-stand movement can be used as a measurement of health 
[25]. Other examples of health conditions the elderly frequently have that can impact transfer capability are declines in muscle strength and flexibility [26]; a smaller range of motion and slower reaction time [27] and a decline in balance function [28], [29]. External factors such as seat height, the use of armrest and foot position can also have major influences on patients' abilities to perform sit-to-stand movements [30]. Alexander et al. studied the effects that chair designs can have on the way that elderly people rise from a chair [31] and Etnyre et al. used a force plate technology to analyze the sit-tostand movement performed by 100 patients using four different arm placements [32].

In the past numerous sit-to-stand movement studies have been performed using a large range of technologies. Kerr et al. analyzed the sit-to-stand cycle performed by 50 normal patients using a vector stereograph, triaxial accelerometers and an electrogoniometer to measure linear displacement in three dimensions as well as the angular displacement at the patients' knee [33]. Lindemann et al. performed a study on 135 older patients using a force plate technology; the goal of the study was to break up the movement into two phases and analyze the timing associated with each phase [34]. Sit-to-stand transfer analysis was performed on 173 senior athletes to compare results between two age classifications, 50-64 years old versus 65 years and older [35]. Lomaglio et al. analyzed the sit-to-stand movement performed by 22 stroke patients to see if muscle strength and weight-bearing symmetry relate to their sit-to-stand performance [36]. Duclose et al. performed a study to see the importance of foot position for 18 stroke patients and 15 control patients [37].

As mentioned before fall risk prevention is a major research field. Najafi et al. measured the sit-to-stand movement using 11 elderly subjects and performed fall risk 
evaluation [38]. Cheng et al. evaluated the sit-to-stand movement for 33 stroke patients and correlated it with falling [39]. Yamada et al. used force plates to measure sit-to-stand movements in young and older subjects in an attempt to link fall prevention to a set of ground force parameters [40].

Other studies have included using video cameras to perform sit-to-stand analysis; examples include studies performed by Ford-Smith, Galli, Allin and most recently Goffredo. Ford-Smith et al. published a study which videotaped the sit-to-stand movement performed by 93 subjects and evaluated the performance based on the movements of four body regions [41]. Galli et al. used an 8 camera system to prove that an experimental setup performed on both healthy subjects and post stroke patients was effective in analyzing the sit-to-stand movement [42]. Allin et al. presented results using a cost effective system consisting of three web cameras to evaluate sit-to-stand performance. Region of interest algorithms were implemented to track the torso region and it was found that elderly individuals rise slower with a less smooth torso movement than young subjects [43]. Goffredo et al. recorded sit-to-stand movements performed by 5 young subjects and 5 older subjects using a video camera. The study presented a markerless computer vision technique used to track specific areas when performing human motion analysis; the results compared extremely well with clinical studies that used marker based systems [44]. 


\subsection{Pressure Sensors}

Pressure sensors are widely used in the field of Biomedical Engineering, particularly when collecting data from humans and human monitoring. Most pressure sensors provide relative measurements which correspond to a force being applied to the sensor rather than a measurement in units such as Pascals [45].

Fiber optical sensors are widely used to measure pressure, vibration, acceleration, temperature, as well as many other parameters from the aerospace and automotive industries. The main advantage to using these types of sensors is the elimination of electricity at the sensor [46]. In the healthcare field, mini fiber optic pressure sensors are often used for invasive measurements of blood pressure [47], [48].

Piezoelectric sensors are used to create tactile sensors which measure applied pressure. The sensors can detect contact between an object and the sensor as well as measure the amount of force that is applied to the sensor surface [49]. Piezoelectrive force sensors convert an applied force to an analog voltage signal using a thin piezoelectric crystal. Piezoresistive force sensors vary their resistance due to the applied force and have a much smaller thickness making them ideal for human tactile sensing applications [50].

Kinotex sensors can be used to measure pressure and displacement using an optical integrating cavity. Figure 2.1 shows an image of the Kinotex sensor manufactured by Canpolar East Inc. The sensor is made up of two optical fibers connecting into a polymer foam material. A simple optical transducer is used to detect changes in light scatter resulting from an external force being applied to the surface [51]. 


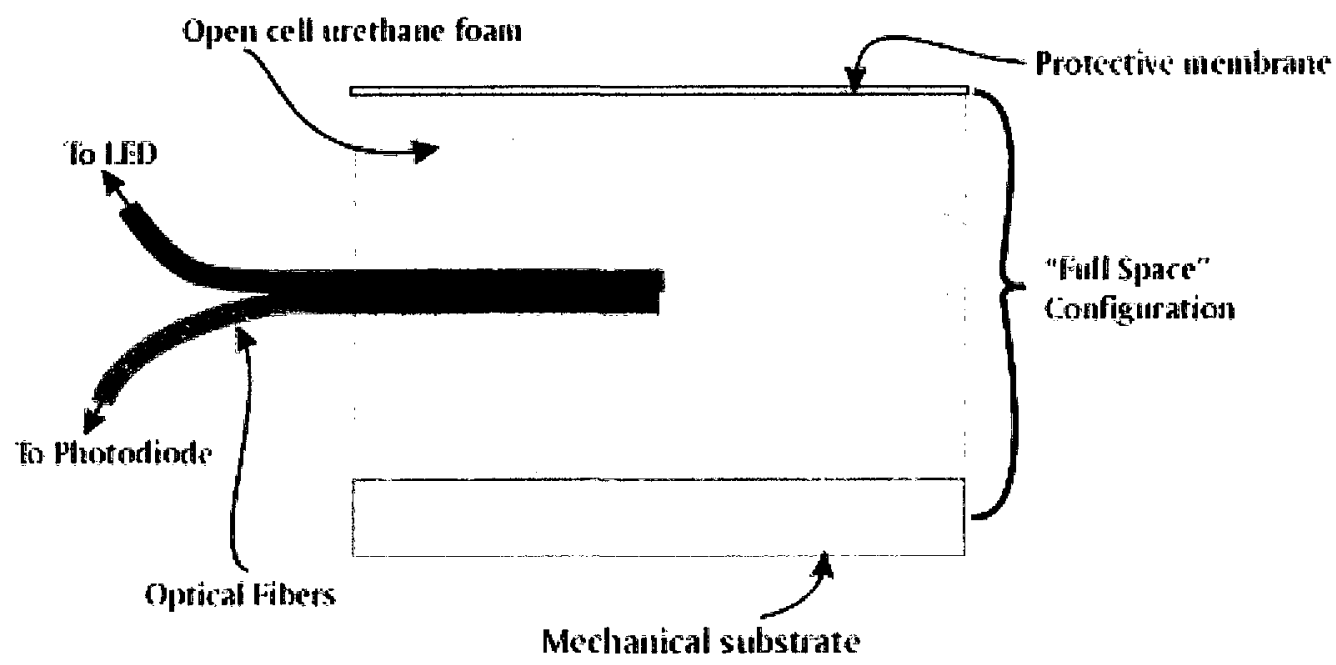

Figure 2.1: KINOTEX ${ }^{\mathrm{TM}}$ Sensor [51]

The main advantage of this technology is the high level of sensitivity, a change of $0.1 \%$ in the total thickness can be detected. This is the same technology that has been adopted by Tactex Controls Inc to create their large pressure array technology which is described in detail in Chapter 3.

Other sensors that can be used to measure pressure are switch sensors which output a zero if no pressure is applied or one if pressure is applied by an external object [52]. Strain gauges, which evaluate the strain that is induced on a structure by an external force, are often used in robotic applications [53].

\subsection{ROI Techniques}

Region of interest (ROI) is a very important concept when performing image processing particularly in medical image analysis. A number of ROI methods exist and are readily used to extract useful information from images. This thesis focuses on implementing thresholding, region labelling, template matching and edge detection methods to extract 
hip and hand regions from pressure images. Each concept will be introduced below and a full description and implementation of each method will be presented in Chapter 5.

Thresholding is often used as a first step when extracting regions from an image; it is used to convert a color or greyscale image into a binary image which can then be processed very quickly using logical operators. Depending on the type of image that is being processed, extracting the objects of interest by choosing a set threshold value can sometimes be a challenge. Threshold values are sometimes chosen based on analyzing a grey-level histogram or otherwise implementing adaptive thresholding algorithms [54], [55].

Binary cluster detection or otherwise called region labelling or connected component identification is useful to identify the location and number of objects of interest in a binary image [56]. Meaningful objects are extracted from a binary image by analyzing and labelling pixels that meet a set of criteria. One technique that exists is the generalized region labelling technique whose advantages include the ability to efficiently perform region labelling using a single pass process, which by avoiding recursion, minimizes the search during labelling [57].

Template matching is an image processing technique which attempts to determine the location of an object by using a template which estimates the approximate size and shape of the object. Several techniques exist for extracting objects when the size and shape parameters are both known and unknown and the basic solution for extracting a known object is to use a whitening filter followed by a matched filter. Other estimators have been suggested such as the phase only matched filter and symmetric phase only matched filter [58]. 
Edge detection is used to segment an image by searching for areas where the image brightness changes sharply and extracting these objects. This results in extracting objects of interest while preserving their border locations [59]. There are many methods for edge detection which can be grouped into two categories, search-based and zero-crossing based. Both methods will be described in detail and implemented using MATLAB in Chapter 5. 


\section{Chapter 3:}

\section{Experiment Setup}

\subsection{Introduction}

This chapter will describe the equipment that was used for data collection as well as the clinical trial that was conducted at the Élisabeth Bruyère hospital. The Mobility project focuses on examining bed transfers using a pressure sensitive mat technology. This project is run by a multidisciplinary team involving engineers, medical clinicians and occupational therapists from Carleton University, the Élisabeth Bruyère hospital and the University of Ottawa.

The following diagram shows the thesis flowchart presented in Chapter 1 with the specific areas covered in Chapter 3 outlined in red. 


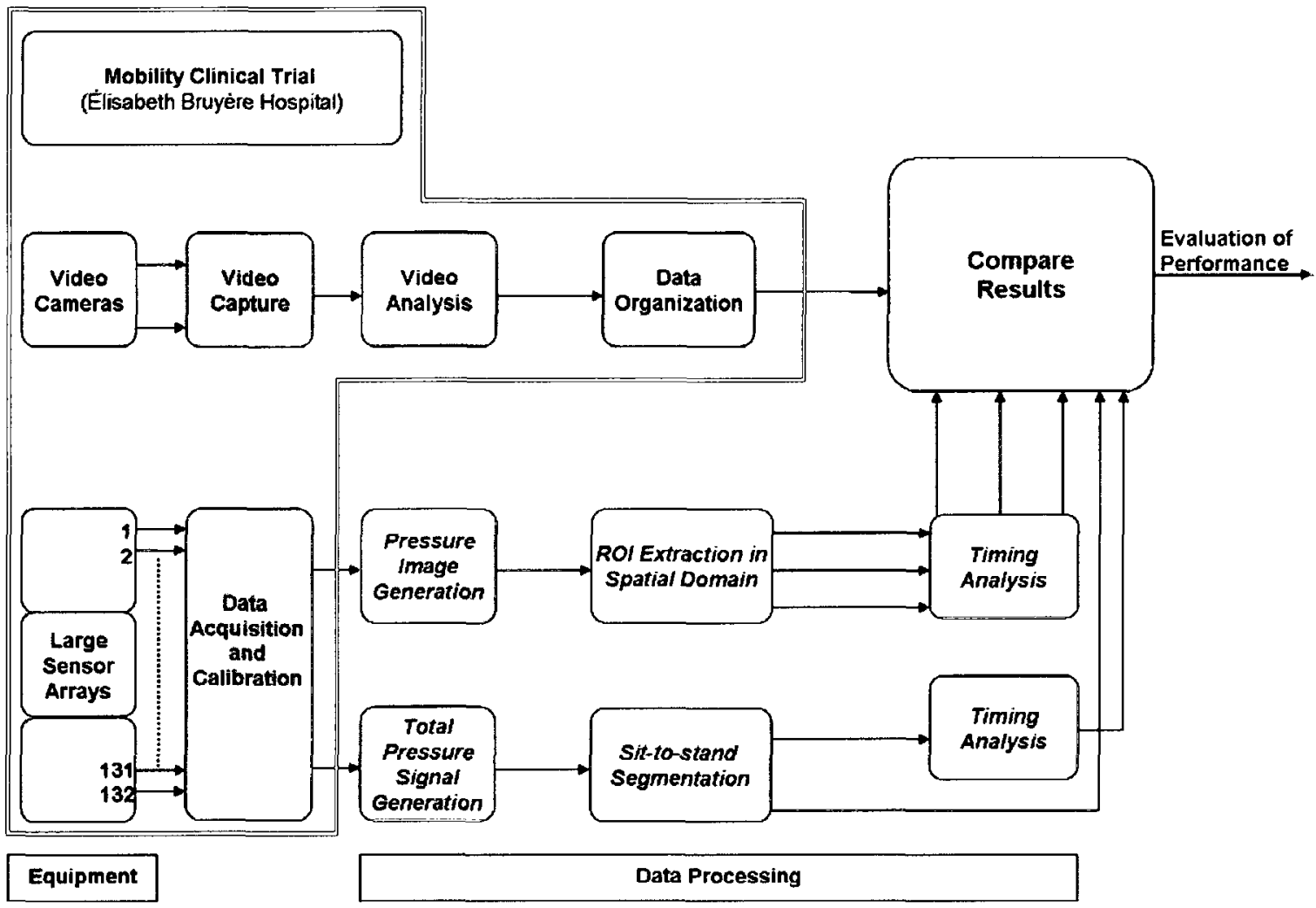

Figure 3.1: Thesis Flowchart

A more detailed Chapter 3 flowchart is shown below.

Mobility Clinical Trial

(Élisabeth Bruyère Hospital)
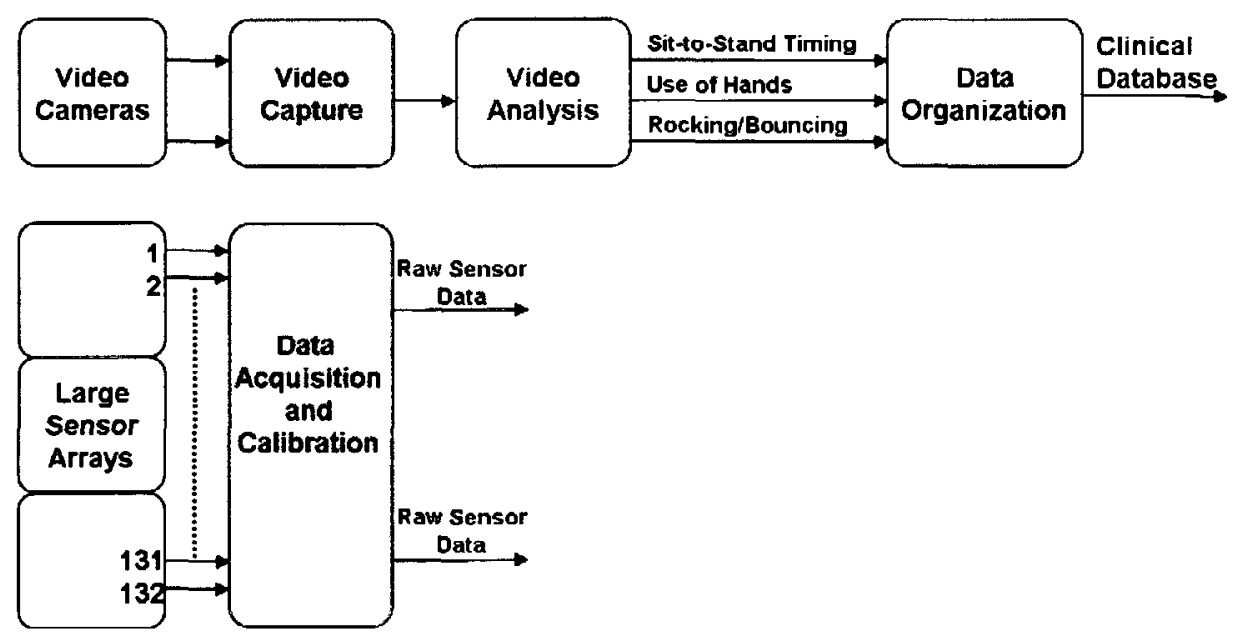

Figure 3.2: Experiment Setup Flowchart 


\subsection{Hardware Equipment}

The sensor array technology used for data collection consists of three Large Sensor Arrays and one High Density Floor Sensor, both manufactured by Tactex Controls Inc. Each sensor is made up of cellular foam with two plastic optical fiber wires, which serve as transmit and receive fibers, embedded into it. The transmit fiber sends light from an LED into the sensor and the receive fiber sends the intensity of the backscattered light back for processing. Relative pressure is the measure of the backscatter light intensity resulting from an external force being applied to the foam sensor. These are the output values for each sensor, which are stored during data acquisition.

\subsubsection{Pressure Sensitive Bed Mats}

Three Large Sensor Arrays were used for bed pressure monitoring. Each mat is $66 \times 71 \mathrm{~cm}$ in size and is made up of a sturdy, flexible foam structure containing 44 embedded fiber optic pressure sensors called "taxels". Each mat is encased in a black polyurethane cover and the three mats were placed side-by-side underneath a bed mattress. Together they covered an area of approximately $66 \times 210 \mathrm{~cm}$ containing 132 taxels.

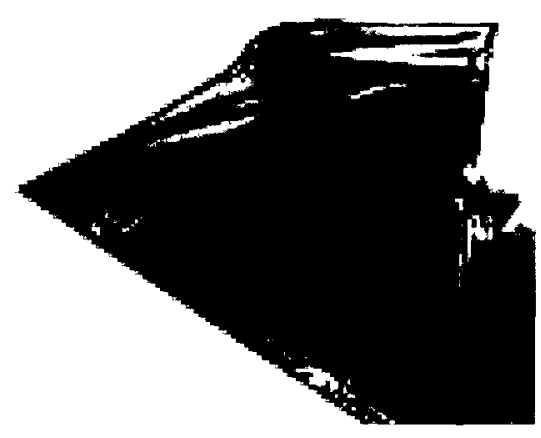

Figure 3.3: Large Sensor Array [60] 


\subsubsection{Pressure Sensitive Floor Mat}

The High Density Floor Sensor was used for floor pressure monitoring. The floor sensor is made up of a durable hard material and is $60 \times 60 \mathrm{~cm}$ in size containing 2048 sensing elements.

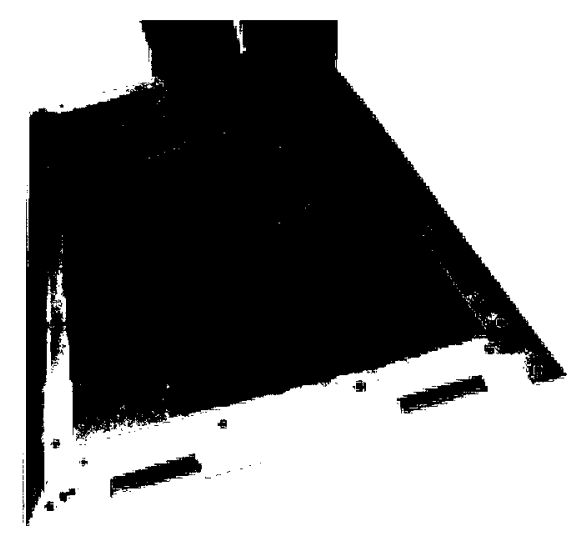

Figure 3.4: High Density Floor Sensor [60]

\subsubsection{Video Cameras}

Two Sony video cameras were used to record participants during the clinical trial. One camera was mounted on a tripod approximately 3 meters in front of the bed and the second camera was mounted on a tripod approximately 3 meters from the side of the bed.

\subsubsection{Equipment Setup}

Both the bed mats and floor mat were installed in the Élisabeth Bruyère hospital to be used for data collection in the clinical trial described in Section 3.4. Figure 3.2 shows a schematic of the equipment setup and Figure 3.3 shows the actual bed mat setup installed in the hospital. 


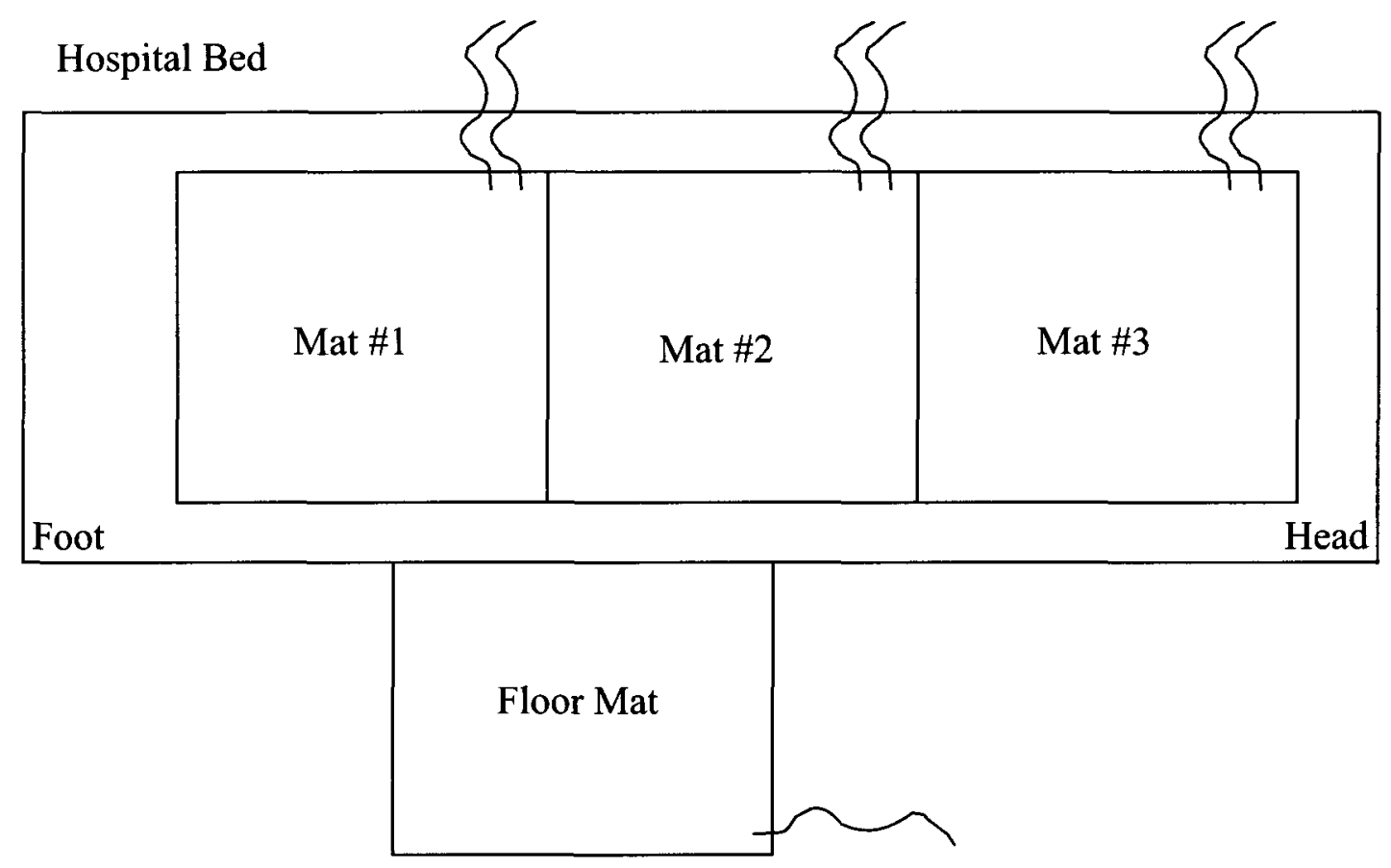

Figure 3.5: Schematic of the equipment setup

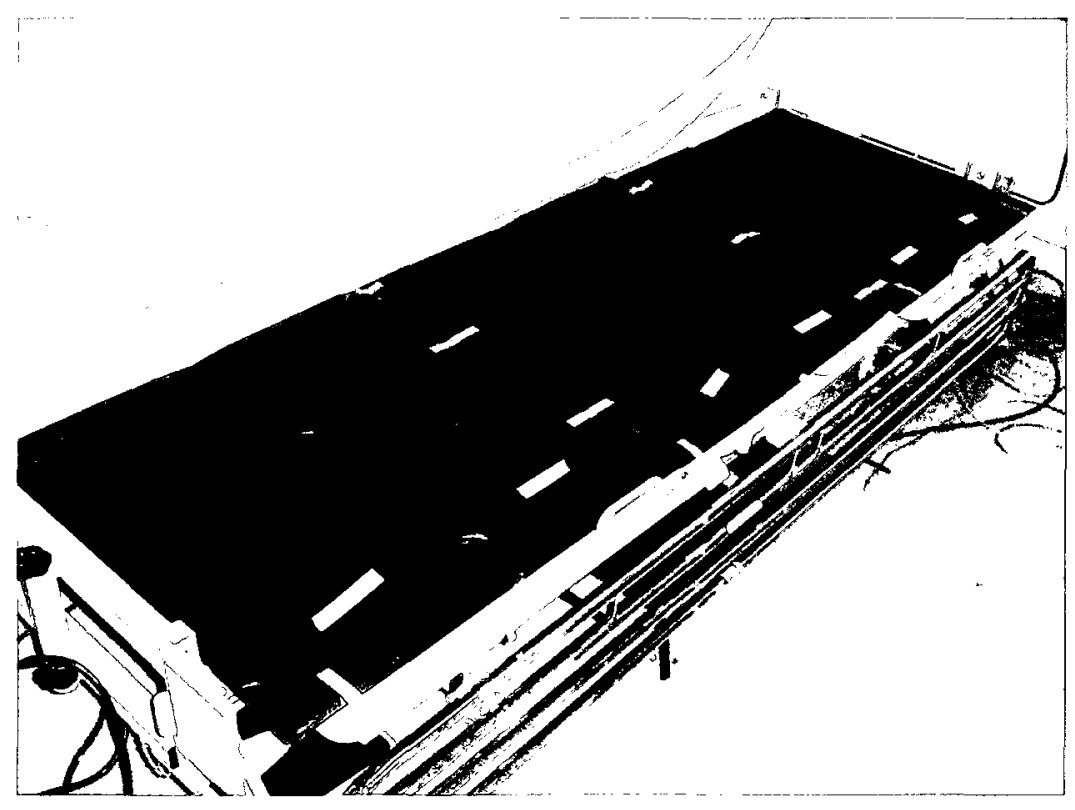

Figure 3.6: Bed mat installation at the Élisabeth Bruyère hospital 
The complete equipment installation at the hospital is shown in Figure 3.4.

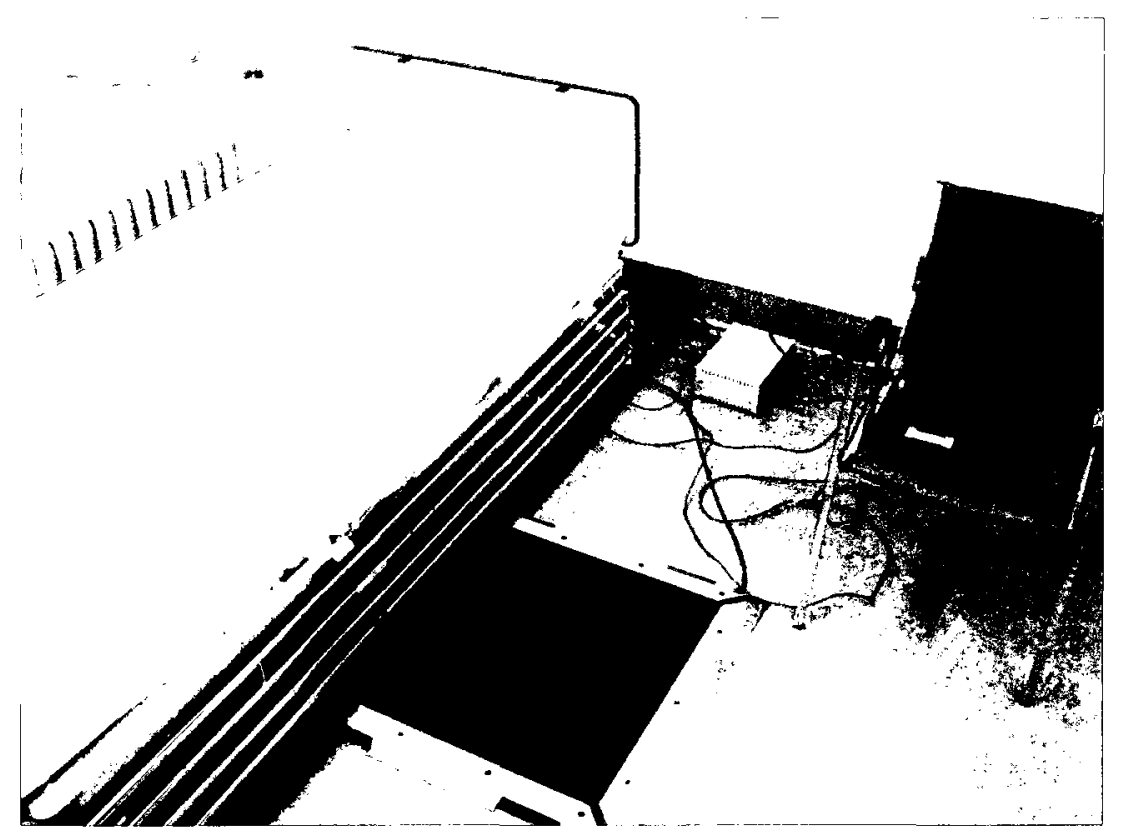

Figure 3.7: Equipment setup installed in the Élisabeth Bruyère hospital

The complete installation at the hospital included the bed mats, floor mat and laptop used for data collection. Preliminary tests were conducted on 10 recruited healthy normal participants to ensure that the equipment installation was working correctly.

A user manual describing the equipment setup process for both the bed mat and floor mat was written and is located in Appendix A.

\subsection{Data Acquisition}

The three mats were connected through an NPort server to a PC laptop using an Ethernet connection. Data was collected from the bed mats and floor mat using a Tactex software interface at a predetermined sampling rate of $10 \mathrm{~Hz}$ for the bed mats and $50 \mathrm{~Hz}$ for the floor mat. The sampling rates differ as a result of the hardware limitations imposed by the manufacturer, Tactex Controls Inc. The data was stored on the laptop computer into 
comma separated files, each column storing data outputs from one taxel. A user manual describing the steps required when collecting data from both the bed mats and floor mat was written and is located in Appendix B.

Simultaneously with the bed and floor data collection, two video cameras were used for recording the participant sit-to-stand transfers from the front view and side view. The occupational therapy team analyzed all of the video recorded sit-to-stand transfers and produced a database of results. The database was created to compare the transfers of the four participant groups using newly developed transfer tools, and to give engineers clinical results to use when comparing and validating their algorithms.

\subsection{Clinical Trial}

The clinical trial included 25 recruited participants and was conducted at the Élisabeth Bruyère hospital. The purpose of the experiment was to analyze the differences between participants while they perform bed transfers. The project was approved by the hospital Research Ethics Board and it was named the Mobility clinical trial. The recruited participants included 10 young healthy subjects, 5 older healthy subjects, 5 post-stroke subjects and 5 post hip-fracture subjects. An occupational therapy student explained the experiment procedure in detail to each participant; following the experiment explanation each participant filled out and signed a consent form. One experiment log sheet was filled out for each subject participating in the clinical trial. Measurements including height, weight, hand width, trunk length, hip width, leg length and foot length were collected and recorded for each participant. After the log sheet was complete each participant began the experiment by performing one test bed transfer. Figure 3.8 outlines the positions which make up one bed transfer sequence. 


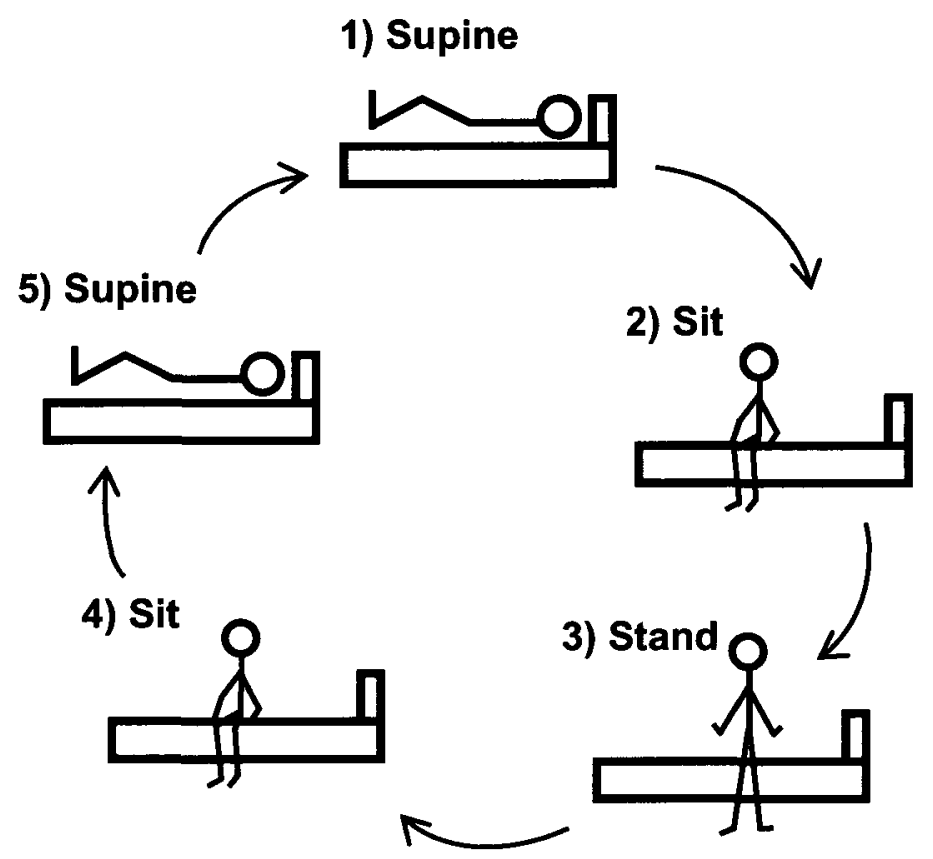

Figure 3.8: Bed transfer sequence

Bed transfers performed by elderly subjects were supervised by either a physical therapist or an occupational therapist, employed by the hospital, to ensure participants' safety. Young healthy and older healthy participants performed 20 bed transfers each while post-hip fracture and post-stroke participants performed 10 bed transfers each. Performing $10-20$ bed transfers consecutively was a difficult task for many subjects; therefore participants experiencing discomfort or fatigue were given breaks between transfers to rest and minimize any chance that they might fall. Data for the Mobility clinical trial was collected from all 25 recruited participants successfully during the summer of 2008. 


\section{Chapter 4:}

\section{Generation of Pressure Images}

\subsection{Introduction}

The following diagram shows the thesis flowchart presented in Chapter 1 with the specific area discussed in Chapter 4 outlined in red.

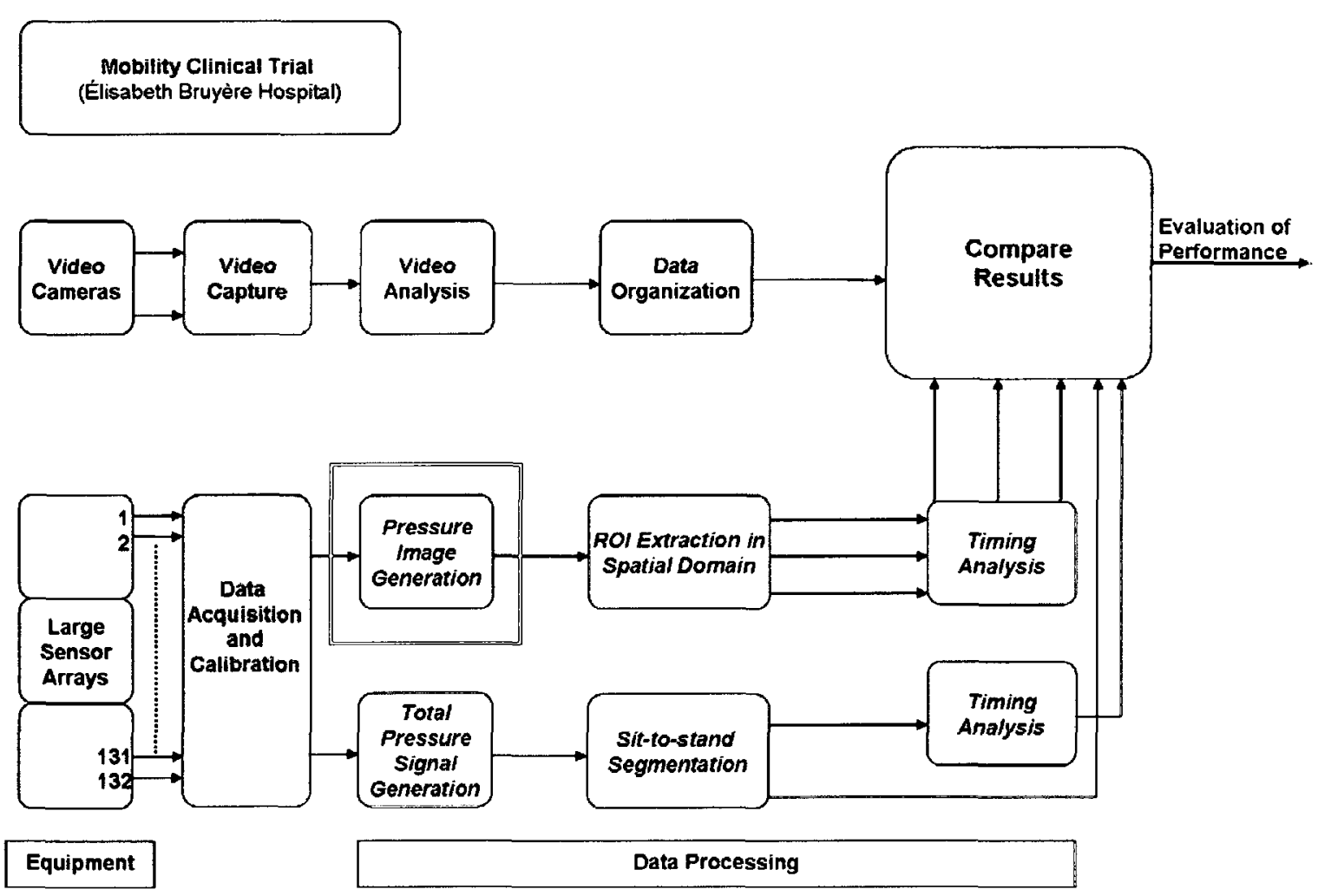

Figure 4.1: Thesis Flowchart 
A more detailed Chapter 4 flowchart is shown below.

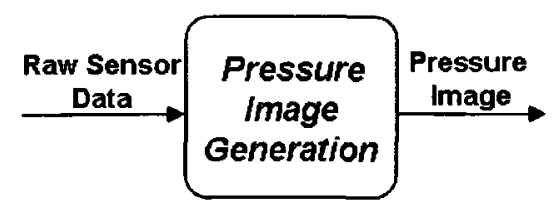

Figure 4.2: Image Generation of Pressure Images Flowchart

The chapter focuses on processing the sit-to-stand data collected during the Mobility clinical trial using the pressure arrays described in Chapter 3 . The main areas of focus include analyzing the data to produce a measure of pressure being applied to the bed as well as the locations of this pressure. In the past a study was performed to help evaluate the optimal grab bar placement for installation into bathrooms [61], [62]. A set of three pressure sensors were used to collect sit-to-stand data from young and older subjects. Two sets of three sensors were installed in two grab bars and the data was analyzed for contact location and force estimation. A set of algorithms for accurate contact location was published by Arcelus et al. [61] whereby the location is estimated based on the sensor array outputs. The force estimation was also calculated using the same sensor array outputs using a set of algorithms published by Holtzman et al. [62]. These approaches worked well when processing data from the sensor array outputs in only 1 dimension (1D); however a different approach will be required when processing the data from the Mobility clinical trial where pressure arrays with a 2 dimensional (2D) sensor arrangement were used. This chapter examines four methods used to create images using the data collected from the pressure sensitive arrays. 


\subsection{Mapping Pressure Sensor Array Data}

The first step of data processing involves mapping the raw data output from the Large Sensor Arrays described in Section 3.2.1. Each value from the 132 pressure sensors is mapped onto an image using its physical $\mathrm{x}$ and $\mathrm{y}$ coordinates. Figure 4.3 shows the locations for all 132 sensors which make up the pressure sensitive arrays that were placed underneath the bed mattress during participant data collection. The sensor numbers and corresponding $\mathrm{x}$ and $\mathrm{y}$ locations were provided by the manufacturers, Tactex Controls Inc.

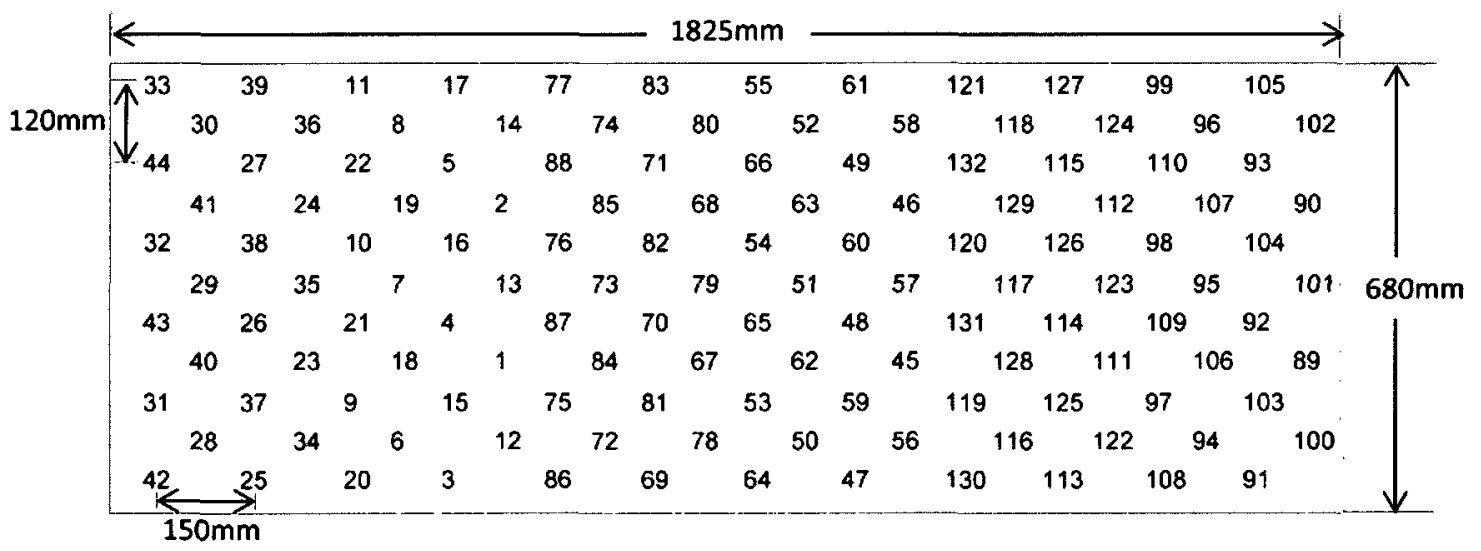

Figure 4.3: Pressure sensor (taxel) locations

The mapping shown above is useful for visualizing the physical locations of the taxels because they are numbered in such a random manner. Taxel outputs are stored in columns in a comma separated file; therefore looking at the file without the taxel mapping makes it very difficult to relate the taxel readings to their physical location on the bed mats.

The large separation in taxel locations greatly reduces the equipment cost therefore making this a feasible technology to be used for installation into older people's homes and elderly care facilities. However in order to produce accurate pressure images from 
the collected data, interpolation methods will be used to overcome the spatial resolution that results from collecting data from a bed surface using 132 pressure sensors.

\subsection{Spatial Interpolation of Sensor Array Data}

Once the raw taxel data has been mapped onto the pressure image, interpolation methods are applied to overcome the spatial separation between the sensors. Increasing the spatial resolution of the image will help with region of interest detection. Four different spatial interpolation methods are presented and their results are compared.

\subsubsection{Interpolation Using Gaussian Kernel Convolution}

The first method used to create pressure images from the raw data begins with the creation of a zero matrix representing the true size of the sensing area of the large sensor array in millimeters using a scale factor of 0.2 . The scale factor is used to reduce processing time for future computations. The 132 taxel values are mapped onto the 365 pixels by 136 pixels zero matrix using their $\mathrm{x}$ and $\mathrm{y}$ coordinates each multiplied first by the 0.2 scale factor. Next $2 \mathrm{D}$ convolution is used to interpolate between the data points in the image. A 2D Gaussian lowpass filter with kernel size 120 by 120 and standard deviation of 20 is used. The image is convolved with the Gaussian kernel and the central part of the resulting matrix is extracted to produce an image of the same original size (365 x 136) with additional data points inserted in between the raw data values. Finally the image is converted to greyscale. The resulting final image dimensions are 365 by 136 with pixel values ranging from zero (black pixels) to one (white pixels). A sample image is shown in Figure 4.4. 


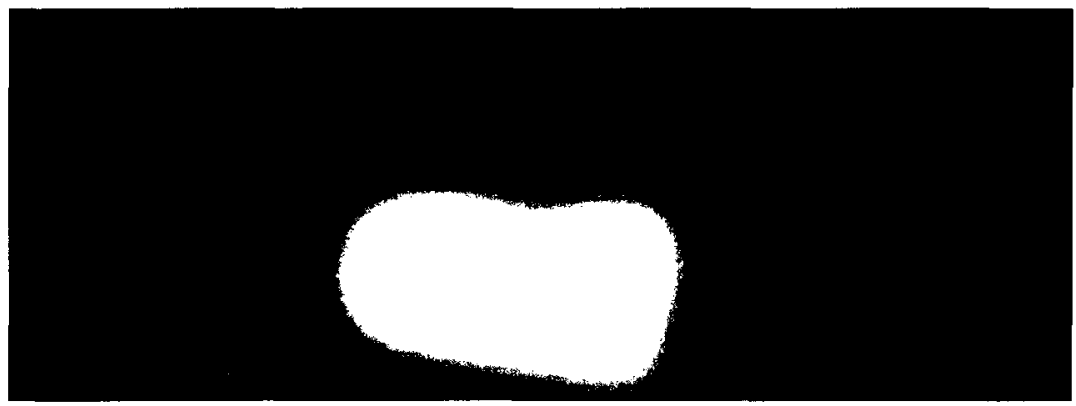

Figure 4.4: Pressure image created using 2D convolution with a Gaussian kernel

\subsubsection{Gaussian Kernel Convolution and Polynomial Interpolation}

The second method used to create pressure images is very similar to the previous one with the difference being the use of a smaller scale factor. A zero matrix representing the true size of the pressure sensitive array scaled by a factor of 0.02 is created. The 132 taxel values are mapped onto the 37 by 14 zero matrix using their corresponding scaled $x$ and $y$ coordinates. Interpolation between the data points is performed using $2 \mathrm{D}$ convolution. A Gaussian lowpass filter with a kernel size of 12 by 12 and standard deviation of 2 is created. The image is convolved with the Gaussian kernel producing interpolated values between the original data points. Next polynomial interpolation is performed using a bicubic interpolator to increase the size of the image by a factor of approximately 10 to a final size of 365 by 136 . Finally the image is converted to greyscale therefore converting each pixel value to a new value between zero (black pixels) to one (white pixels).

\subsubsection{Linear Interpolation}

Linear interpolation is the third method used to create pressure images and it differs greatly from the two methods described above. The main goal of this technique is to reduce the number of zeros inserted between the taxel data location when performing the initial taxel data mapping. 
The first step in creating pressure images using this method is to create two 24 by 11 zero matrices. The taxel data values are mapped onto each matrix using a checkerboard pattern. The actual $\mathrm{x}$ and $\mathrm{y}$ mapping coordinates are ignored and instead an equal separation between taxels is assumed. A sample matrix is shown in Figure 4.5; the X's represent the taxel data values.

\begin{tabular}{|c|c|c|c|c|c|c|c|c|c|c|c|c|c|c|c|c|c|c|c|c|c|c|c|}
\hline $\mathrm{X}$ & & $\mathrm{X}$ & & $\mathrm{X}$ & & $\mathrm{X}$ & & $\mathrm{X}$ & & $\mathrm{X}$ & & $\mathrm{X}$ & & $\mathrm{X}$ & & $\mathrm{X}$ & & $\mathrm{X}$ & & $\mathrm{X}$ & & $\mathrm{X}$ & \\
\hline & $\mathrm{X}$ & & $\mathrm{X}$ & & $\mathrm{X}$ & & $\mathrm{X}$ & & $\mathrm{X}$ & & $\mathrm{X}$ & & $\mathrm{X}$ & & $\mathrm{X}$ & & $\mathrm{X}$ & & $\mathrm{X}$ & & $\mathrm{X}$ & & $\mathrm{X}$ \\
\hline $\mathrm{X}$ & & $\mathrm{X}$ & & $\mathrm{X}$ & & $\mathrm{X}$ & & $\mathrm{X}$ & & $\mathrm{X}$ & & $\mathrm{X}$ & & $\mathrm{X}$ & & $\mathrm{X}$ & & $\mathrm{X}$ & & $\mathrm{X}$ & & $\mathrm{X}$ & \\
\hline & $\mathrm{X}$ & & $\mathrm{X}$ & & $\mathrm{X}$ & & $\mathrm{X}$ & & $\mathrm{X}$ & & $\mathrm{X}$ & & $\mathrm{X}$ & & $\mathrm{X}$ & & $\mathrm{X}$ & & $\mathrm{X}$ & & $\mathrm{X}$ & & $\mathrm{X}$ \\
\hline $\mathrm{X}$ & & $\mathrm{X}$ & & $\mathrm{X}$ & & $\mathrm{X}$ & & $\mathrm{X}$ & & $\mathrm{X}$ & & $\mathrm{X}$ & & $\mathrm{X}$ & & $\mathrm{X}$ & & $\mathrm{X}$ & & $\mathrm{X}$ & & $\mathrm{X}$ & \\
\hline & $\mathrm{X}$ & & $\mathrm{X}$ & & $\mathrm{X}$ & & $\mathrm{X}$ & & $\mathrm{X}$ & & $\mathrm{X}$ & & $\mathrm{X}$ & & $\mathrm{X}$ & & $\mathrm{X}$ & & $\mathrm{X}$ & & $\mathrm{X}$ & & $\mathrm{X}$ \\
\hline $\mathrm{X}$ & & $\mathrm{X}$ & & $\mathrm{X}$ & & $\mathrm{X}$ & & $\mathrm{X}$ & & $\mathrm{X}$ & & $\mathrm{X}$ & & $\mathrm{X}$ & & $\mathrm{X}$ & & $\mathrm{X}$ & & $\mathrm{X}$ & & $\mathrm{X}$ & \\
\hline & $\mathrm{X}$ & & $\mathrm{X}$ & & $\mathrm{X}$ & & $\mathrm{X}$ & & $\mathrm{X}$ & & $\mathrm{X}$ & & $\mathrm{X}$ & & $\mathrm{X}$ & & $\mathrm{X}$ & & $\mathrm{X}$ & & $\mathrm{X}$ & & $\mathrm{X}$ \\
\hline $\mathrm{X}$ & & $\mathrm{X}$ & & $\mathrm{X}$ & & $\mathrm{X}$ & & $\mathrm{X}$ & & $\mathrm{X}$ & & $\mathrm{X}$ & & $\mathrm{X}$ & & $\mathrm{X}$ & & $\mathrm{X}$ & & $\mathrm{X}$ & & $\mathrm{X}$ & \\
\hline & $\mathrm{X}$ & & $\mathrm{X}$ & & $\mathrm{X}$ & & $\mathrm{X}$ & & $\mathrm{X}$ & & $\mathrm{X}$ & & $\mathrm{X}$ & & $\mathrm{X}$ & & $\mathrm{X}$ & & $\mathrm{X}$ & & $\mathrm{X}$ & & $\mathrm{X}$ \\
\hline $\mathrm{X}$ & & $\mathrm{X}$ & & $\mathrm{X}$ & & $\mathrm{X}$ & & $\mathrm{X}$ & & $\mathrm{X}$ & & $\mathrm{X}$ & & $\mathrm{X}$ & & $\mathrm{X}$ & & $\mathrm{X}$ & & $\mathrm{X}$ & & $\mathrm{X}$ & \\
\hline
\end{tabular}

Figure 4.5: Taxel mapping used to create linear interpolation pressure images

Once the matrices have been filled 1D linear interpolation is performed along the rows of the first matrix and along the columns of the second matrix. The blank values shown in Figure 4.5 are replaced with interpolated values calculated from fitting a line through the entire column or row which is made up of the $\mathrm{X}$ values only. The inserted values depend on the neighbouring taxel values in the $\mathrm{x}$ direction for matrix one and the $\mathrm{y}$ direction for matrix two. The two matrices are then averaged to produce a final matrix which takes into account interpolation in the $\mathrm{x}$ and $\mathrm{y}$ direction simultaneously. At this point the images are very small and the difference between the pixel intensities is very large. Next the image is blurred using $2 \mathrm{D}$ convolution with a Gaussian lowpass filter. The kernel size is 5 by 5 and the standard of deviation is 2 . This helps smooth out the pressure 
data but the image is still only 24 by 11 pixels. Next polynomial interpolation is performed using a bicubic interpolator to increase the size of the image to match $20 \%$ of the true size of the pressure mats, a size of 365 by 136. The resizing method is the same as the one used in the previous section. Finally the image is converted to grey scale where individual pixel values are set to new values between zero (black pixels) and one (white pixels).

\subsubsection{Spline Interpolation}

The fourth method presented for pressure image creation is the use of a cubic spline interpolator rather than linear interpolator when creating pressure images using the same steps outlined in Section 4.3.3. The taxel values are mapped onto two 24 by 11 matrices using one to one mapping as shown in Figure 4.5. Next 1D cubic spline interpolation is performed along the rows of the first matrix and the columns of the second matrix. The two matrices are summed and averaged producing a matrix of size 24 by 11 with inserted interpolated values based on both the $\mathrm{x}$ and $\mathrm{y}$ direction. Polynomial interpolation is performed to increase the size of the image to 365 by 136 and the entire image is converted to greyscale. A more detailed explanation of these individual steps is described in the previous Section 4.3.3. 


\subsection{Results}

A comparison of pressure images created by the four different processing techniques will be compared using two data sets. A frame from the control participant data will be compared first followed by a comparison of frames from the Mobility clinical trial data.

\subsubsection{Control Participant Results}

One frame from the control participant data was processed using the four techniques explained in Section 4.3 and the results are shown in Figure 4.6.

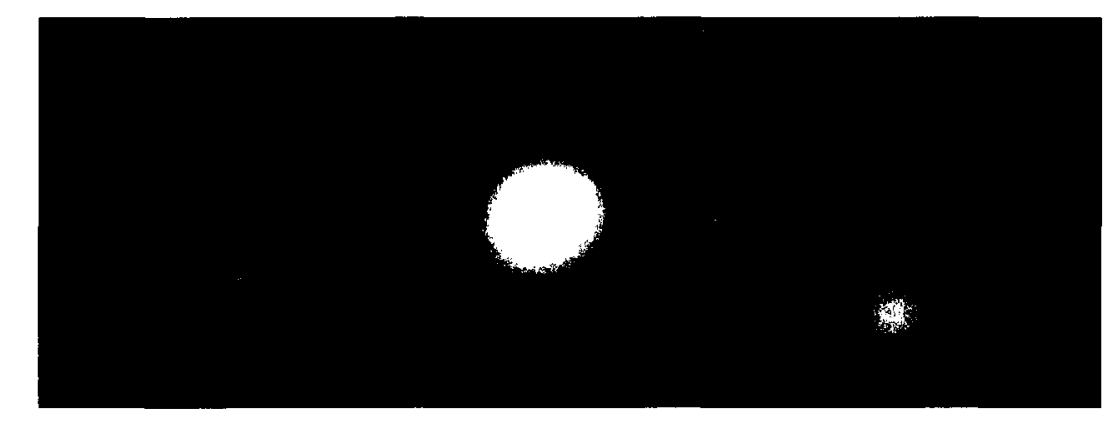

(a) 2D convolution with a Gaussian kernel using a scale factor of 0.2

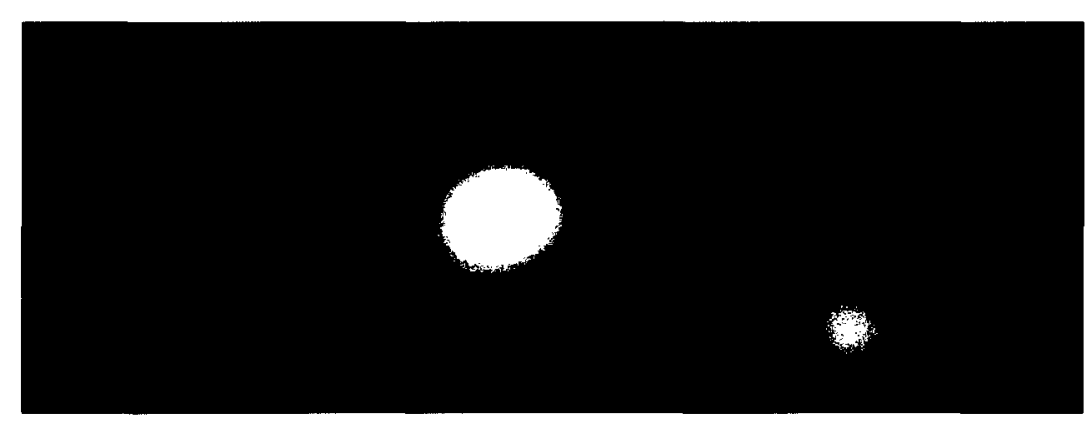

(b) 2D convolution with a Gaussian kernel using a scale factor of 0.02 and polynomial interpolation 


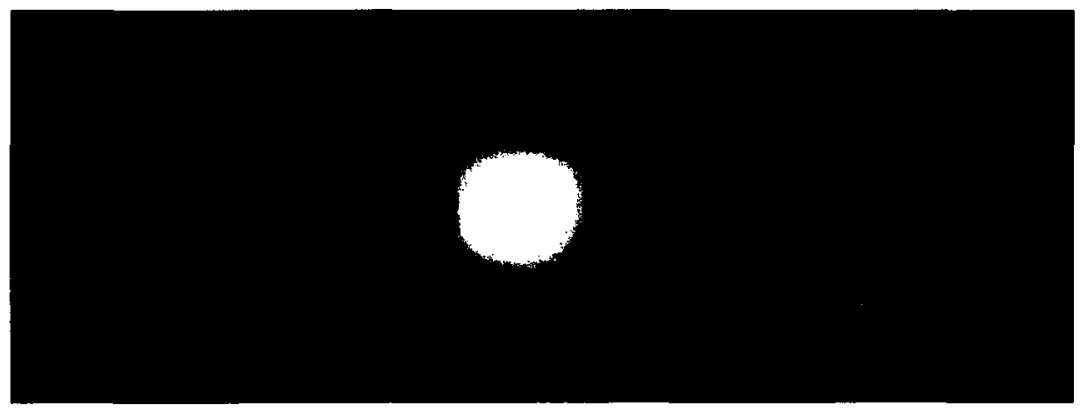

(c) Linear interpolation

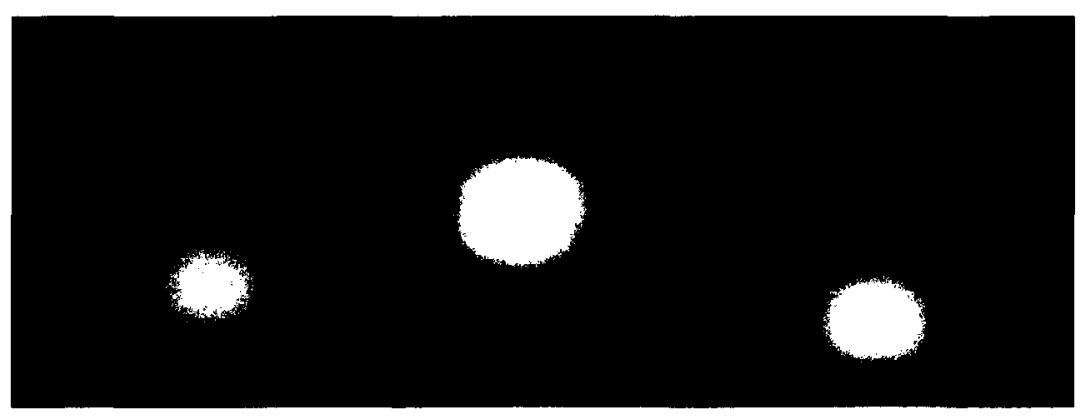

(d) Spline interpolation

Figure 4.6: Comparison of four techniques used to create pressure images representing control participant data

All four techniques produce similar results when applied to the control participant data. This is to be expected because the control participant data represents the ideal data set and can therefore be processed to produce correct results using various techniques.

\subsubsection{Mobility Participant Results}

Two data samples were processed from the mobility participant data using the four techniques described in Section 4.3 to help show differences between the resulting pressure images. The goal of the pressure image is to create an accurate representation of the pressure being applied to the bed. Therefore images showing the hip and hand regions with the greatest amount of detail would provide the best results. The results for two data samples will be presented; a comparison of the four different image construction 
techniques will be performed, and the technique producing the most accurate pressure images will be identified.

The first data sample is an example of a participant sitting on the bed and applying pressure with their right hand; the results are shown in Figure 4.7.

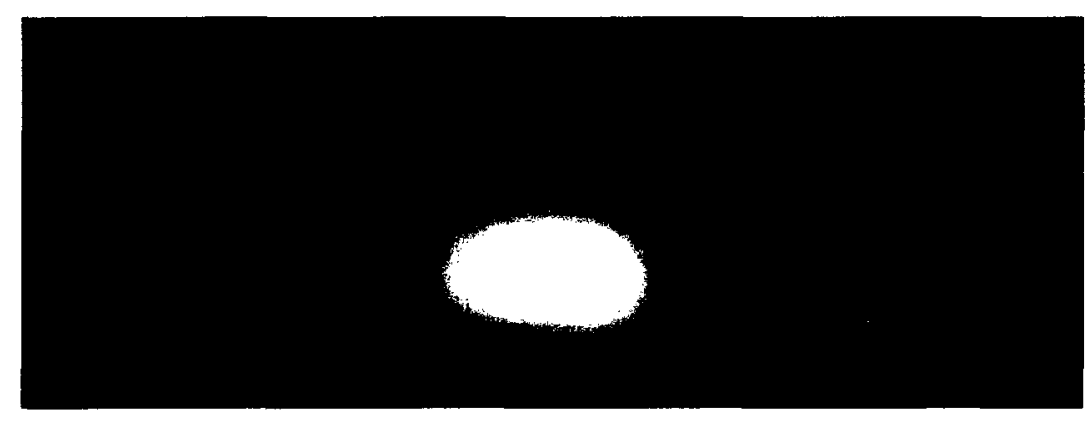

(a) 2D convolution with a Gaussian kernel using a scale factor of 0.2

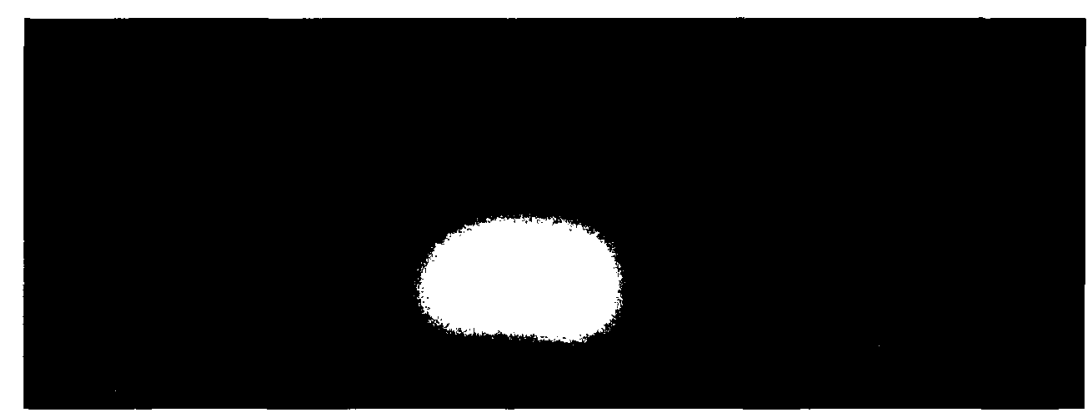

(b) 2D convolution with a Gaussian kernel using a scale factor of 0.02 and polynomial interpolation

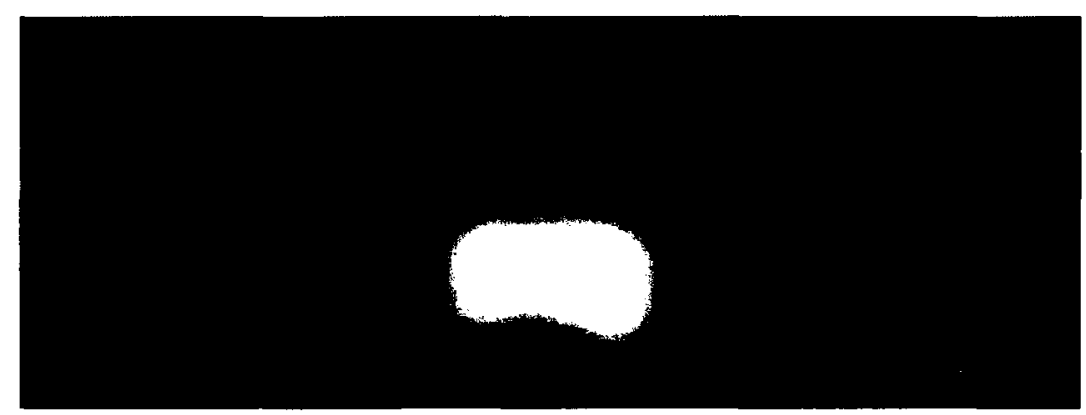

(c) Linear interpolation 


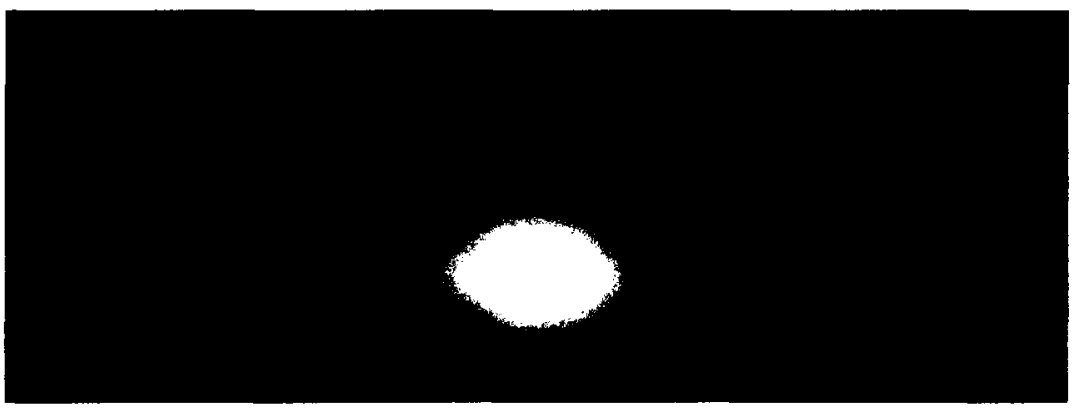

(d) Spline interpolation

Figure 4.7: Comparison of four techniques used to create pressure images representing a seated participant who is pushing on the bed with their right hand

The second data sample is an example of a participant sitting on the bed and applying pressure with their left hand; the results are shown in Figure 4.8.

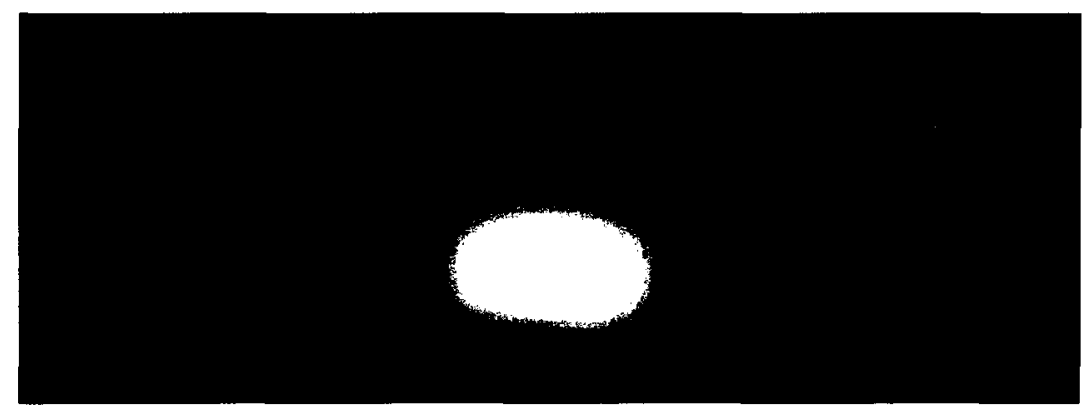

(a) 2D convolution with a Gaussian kernel using a scale factor of 0.2

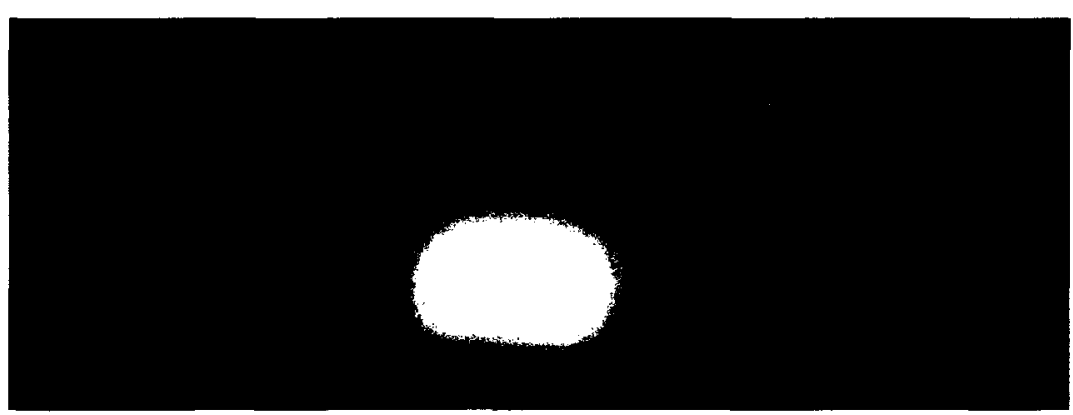

(b) 2D convolution with a Gaussian kernel using a scale factor of 0.02 and polynomial interpolation 


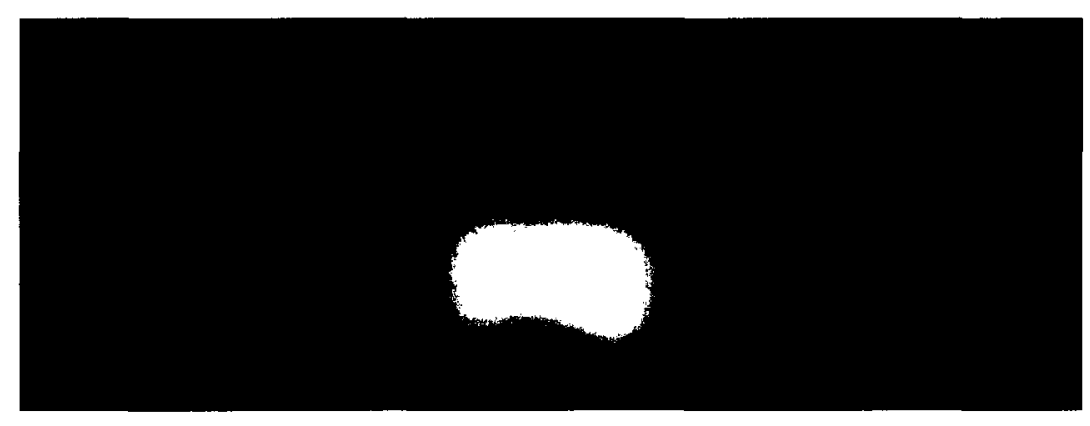

(c) Linear interpolation

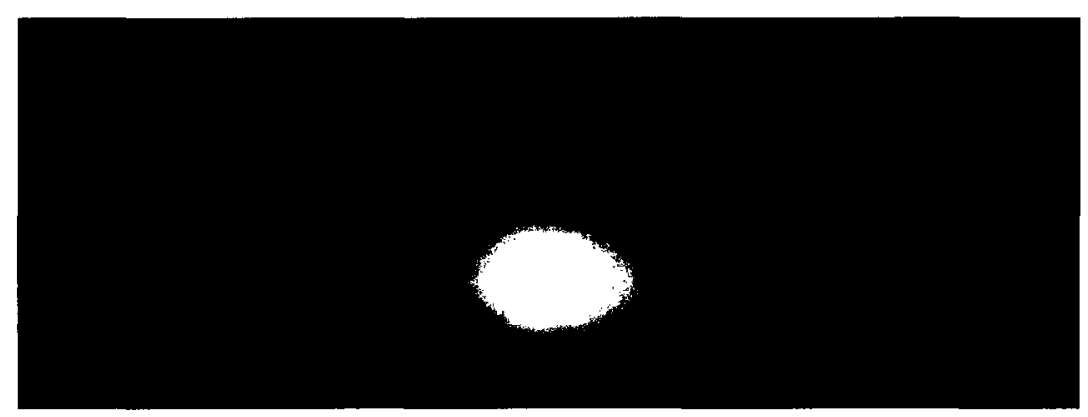

(d) Spline interpolation

Figure 4.8: Comparison of four techniques used to create pressure images representing a seated participant who is pushing on the bed with their left hand

The pressure images shown above give a good approximation of the pressure being applied by a participant to the bed. Differences in the pressure images result from the techniques used to produce them; a brief explanation for each result is presented.

A problem with creating the pressure images using the first technique, Figure 4.7 (a) and Figure 4.8 (a), is that a large number of zeros are used in the first steps to ensure that the taxel data is placed in the correct physical location. These zeros however cause problems when performing spatial interpolation by requiring the use of a filter with a very large kernel size to overcome the large gaps in data throughout the image. This can result in high taxel data values dominating large regions of the pressure images.

The Figure 4.7 (b) and Figure 4.8 (b), images were created using a very similar technique to the previous one, described in Section 4.3.1, and therefore similar problems 
exist. The filter size used to interpolate between the taxel data is big compared to the size of the image therefore high taxel data values will dominate large areas of the pressure image.

The pressure images presented in Figure 4.7 (c) and Figure 4.8 (c) both show a good representation of the force being applied by the participant to the bed. The pressure image is less smooth than the ones shown in Figure 4.7 (a) and (b) and Figure 4.8 (a) and (b), but they give a better representation of the location of the pressure being applied to the bed. Figure 4.7 (c) shows the hip and right hand regions and Figure 4.8 (c) shows the hip and left hand regions. Comparing results from the different techniques, it is clear that these images display the greatest amount of detail, visibly showing the hip and hand regions.

The Figure 4.7 (d) and Figure 4.8 (d) pressure image is a poor representation of the pressure being applied to the bed. The pressure image lacks in detail when compared to the results shown in Figure 4.7 (c) and Figure 4.8 (c) where linear interpolation is used. The problem with this method is the use of a cubic spline interpolator which attempts to fit a perfect curve to a data set but only achieves this if the data set is cubic. The pressure data is not cubic and the difference between adjacent taxel values can vary greatly. Some areas of the bed will display zero and others will display high values because the participant only applies pressure on a certain area of the bed while performing the sit-tostand transfer. Since the cubic spline interpolation is being applied to an entire row or column of data at one time, inaccurate results will be produced due to the many zeros that will be present near the edges. 
Therefore the linear interpolation method produced the most accurate pressure image. This method would be best suited for creating pressure images from raw data which could then be processed further using additional image processing techniques such as region of interest extraction.

\subsection{Conclusion}

Four techniques were presented for creating pressure images from raw taxel data. Sequences of five images extracted from one participants' sit-to-stand transfer were used to compare the four different algorithms. The results presented at the end of each method description show that the linear interpolation technique shown in Section 4.3.3 is the best. The linear method is superior to the spline method because the row and column taxel value data does not fit a cubic model and is therefore interpolated more accurately using linear interpolation. This method uses linear interpolation first to fill in the areas of interest between the taxel values and then applies a lowpass filter to smooth this data together. This initial interpolation allows for a smaller filter size to be used therefore ensuring that high taxel values located in one region of the bed will not dominate other regions in the corresponding pressure image. The two Gaussian convolution methods both rely only on lowpass filtering to perform both the interpolation and smoothing between the taxel value data therefore require the use of large filters. The linear method uses a filter with an area covering approximately $10 \%$ of the image rather than $30 \%$ which is used by both Gaussian interpolation methods. Each of the four techniques presented in this chapter processed 132 taxel data values to produce pressure images; however the technique presented in Section 4.3.3, Linear Interpolation, produced the best results. 


\section{Chapter 5:}

\section{ROI Extraction in Spatial Domain}

\subsection{Introduction}

The following diagram shows the thesis flowchart presented in Chapter 1 with the specific areas covered in Chapter 5 outlined in red.

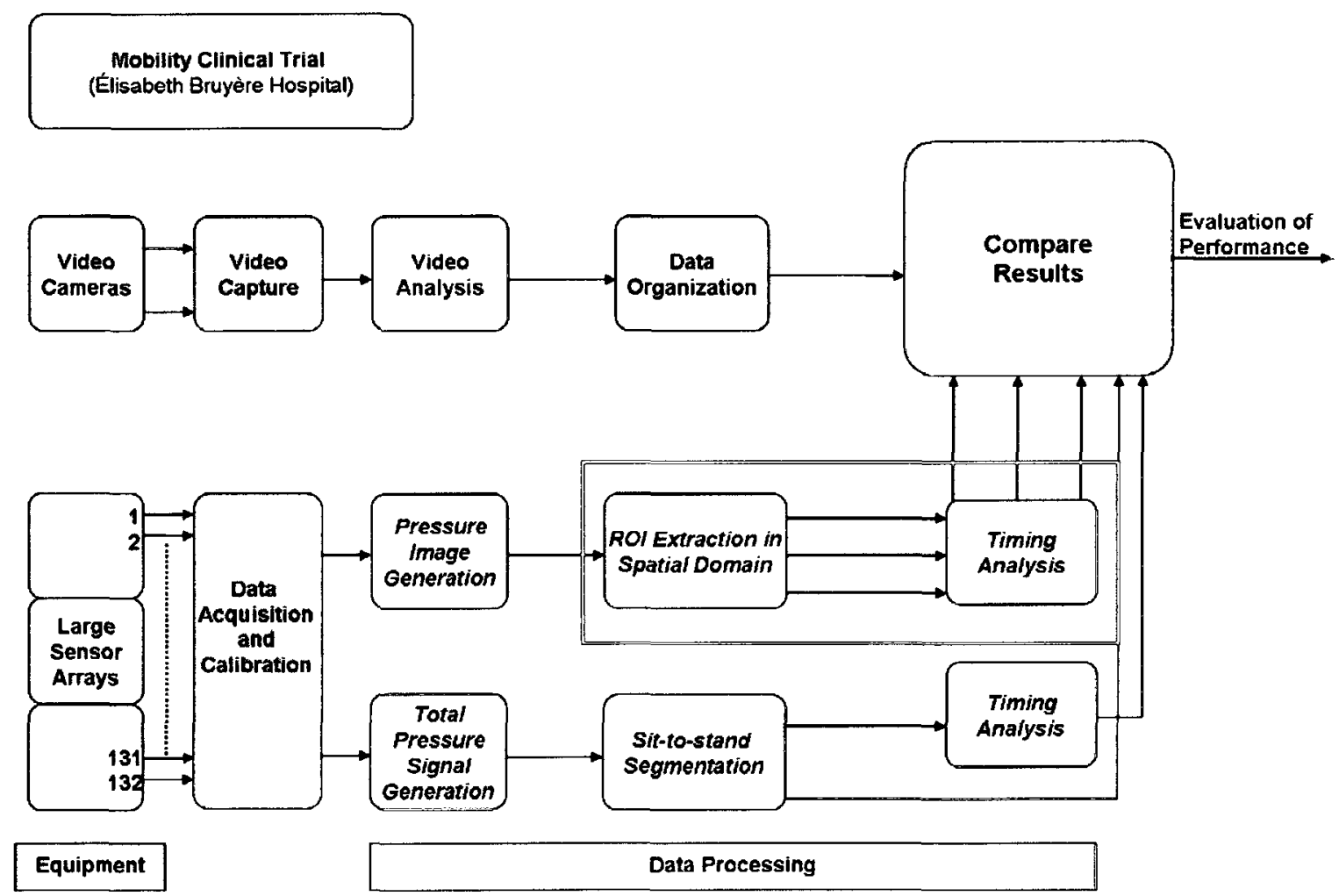

Figure 5.1: Thesis Flowchart 
A more detailed Chapter 5 flowchart is shown below.

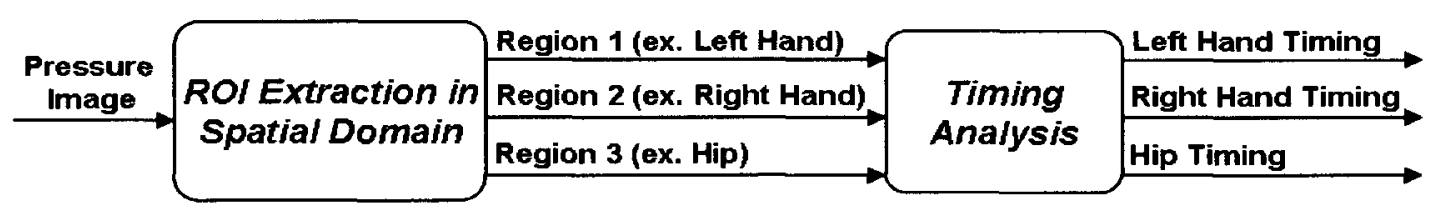

Figure 5.2: Region of Interest Extraction in Spatial Domain Flowchart

This chapter will focus on examining the ideal sit-to-stand sequence of images created by using the method described in Chapter 4 . The goal of this chapter is to perform a frame by frame analysis and extract the hip and hand regions. Four different region of interest algorithms will be described and evaluated. Results from each algorithm will be shown as well as a discussion on the challenges and limitations affecting each algorithm. The three regions of interest are the hip, left hand and right hand regions and they will be labelled as Hip, $\mathrm{LH}$, and $\mathrm{RH}$ throughout the figures in this chapter.

\subsection{Ideal Sit-to-stand Transfer}

The ideal sit-to-stand sequence performed by a control participant is shown in Figure 5.3 below.
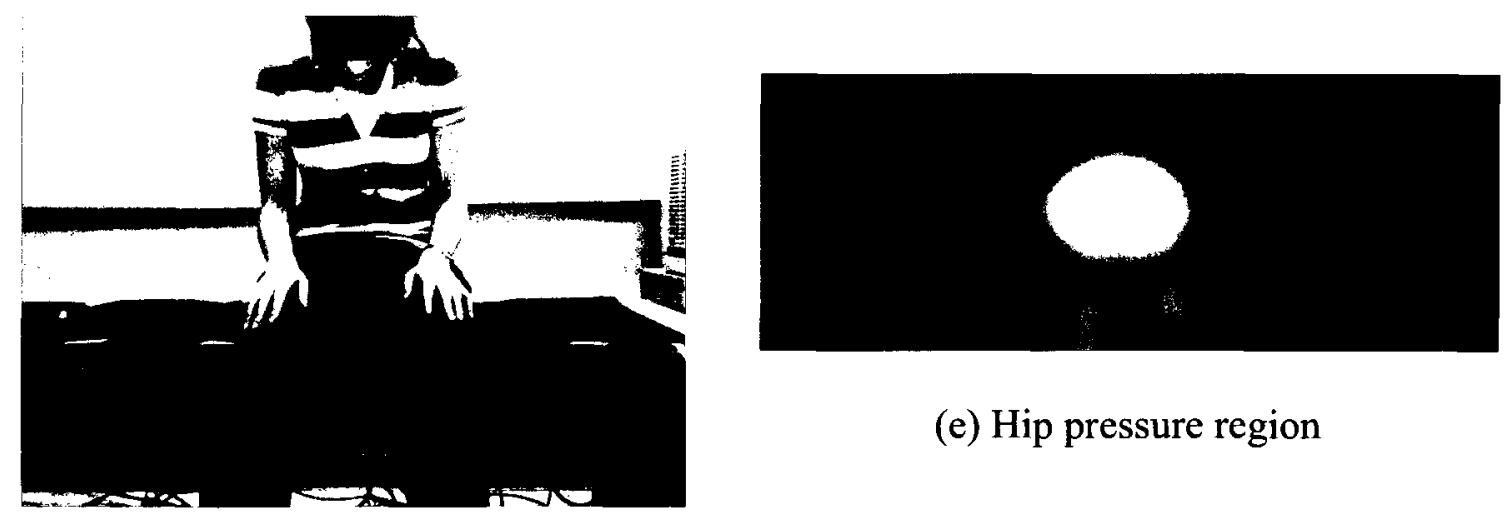

(e) Hip pressure region

(a) Start of bed exit position 


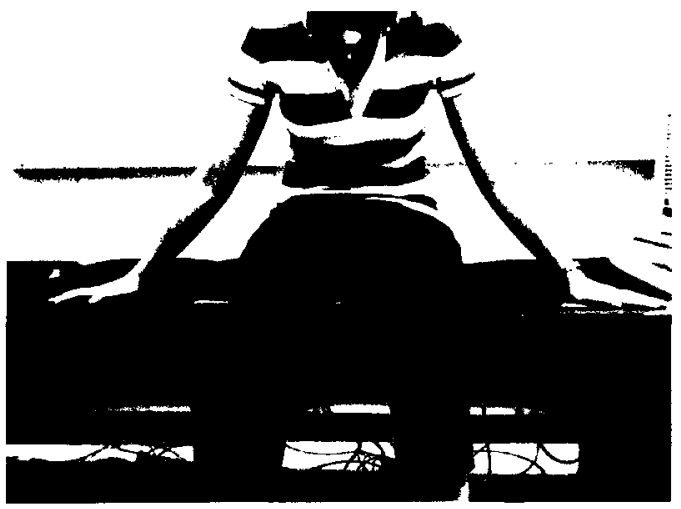

(b) Transition bed exit position, hands apply pressure; pressure transfers from the hips to the hands as the hips begin to lift off

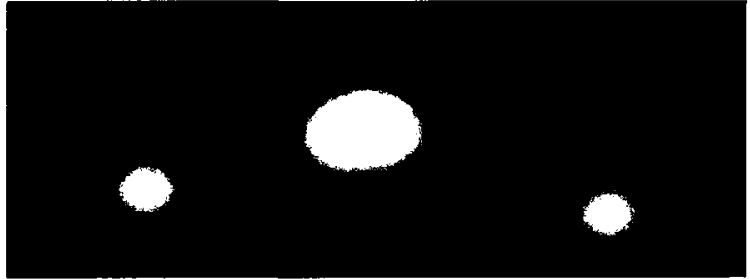

(f) Hip and light hand pressure regions

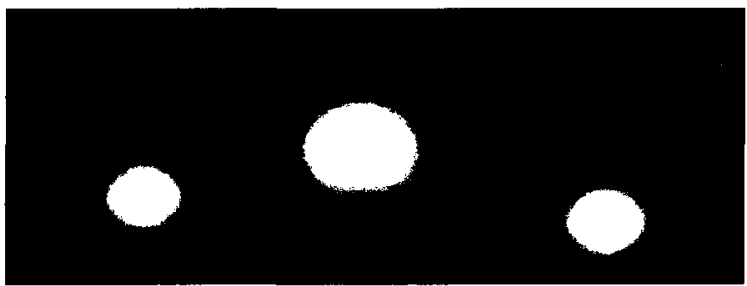

(g) Hip and hand pressure regions

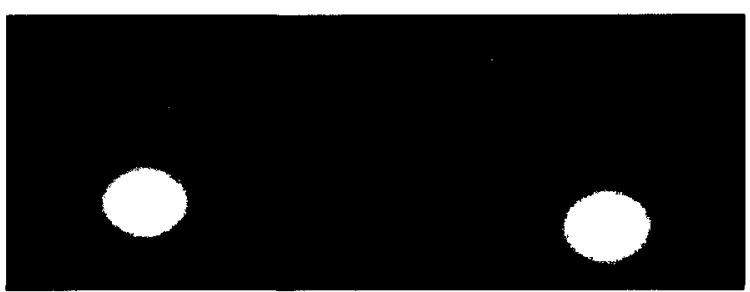

(h) Light hip and strong hand pressure regions

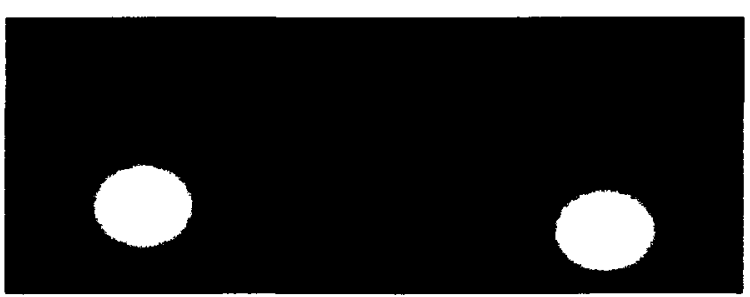

(i) Hand pressure regions

(c) End of lifting phase, all of the pressure is being applied by the hands 


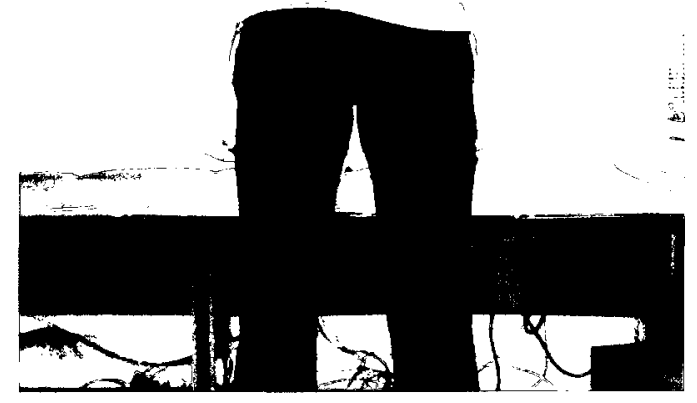

(d) End of bed exit position

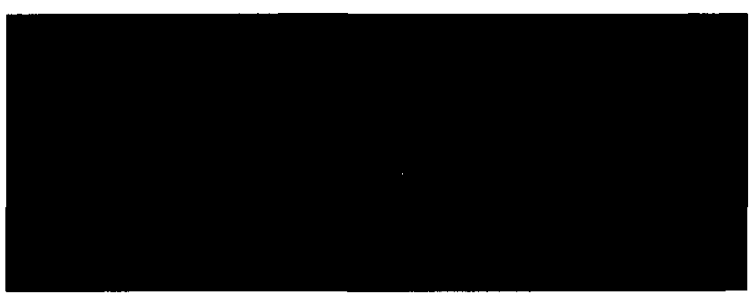

(j) No pressure - bed exit completed

Figure 5.3: Sit-to-stand sequence

The start, lift and end bed exit positions are demonstrated using a test subject in Figure 5.3 parts (a)-(d) while the corresponding pressure images are displayed in Figure 5.3 (e)-(j). Each transfer begins with the subject seated quietly on the bed therefore applying a pressure on the bed corresponding only to their hip region (e). Once the subject is ready to transition to the standing position they apply a small amount of pressure using their hands (f) and continue increasing this hand pressure while reducing their hip pressure (f)-(h). Next the subject lifts their entire hip region off of the bed applying only pressure using their hands (i) until they come to a complete stand position and zero pressure is registered on the bed $(j)$. The remainder of this chapter will look at extracting the hip and hand regions from the sequence of pressure images shown in Figure $5.3(\mathrm{e})-(\mathrm{j})$. 


\subsection{ROI Algorithms}

Four region of interest algorithms are presented and compared for accurate hip and hand region detection.

\subsubsection{Manually Defined Regions}

The first region detection algorithm is based on sectioning the bed into three equal regions which are defined by spatial location. The assumptions this algorithm takes into consideration is that during the ideal sit-to-stand sequence the hips are located in the middle section of the bed, the left hand is located in the far region to the left of the hips at the head of the bed and the right hand is located in the far region to the right of the hips at the foot of the bed. These three regions and their boundaries are shown in Figure 5.4 below.

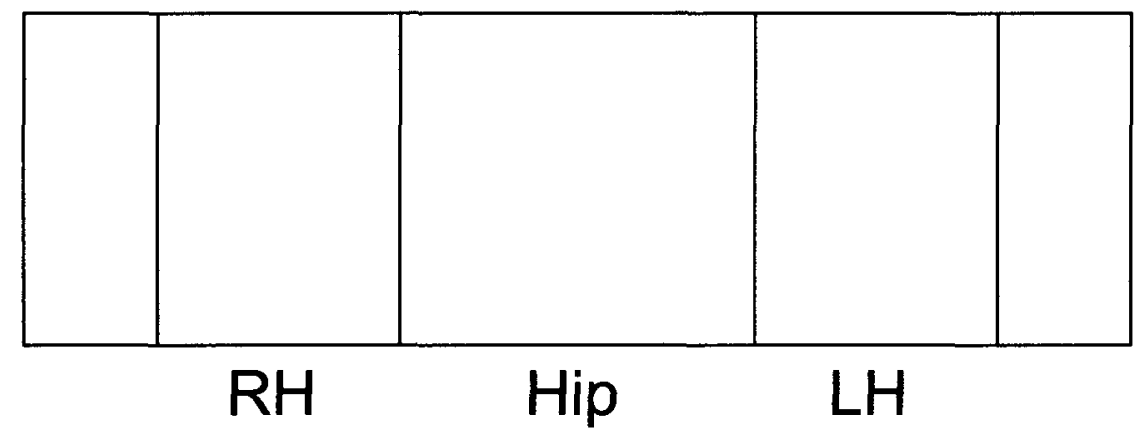

Figure 5.4: The three fixed regions

Once the boundaries of the three regions have been defined individual images are processed by summing the pixel values located in each of the three regions defined. These relative pressures are normalized by the maximum pressure that is calculated removing any participant specific weight dependencies and each value is plotted over time. The results for three different sit-to-stand sequences are displayed in Figure 5.5 Figure 5.7 below. 


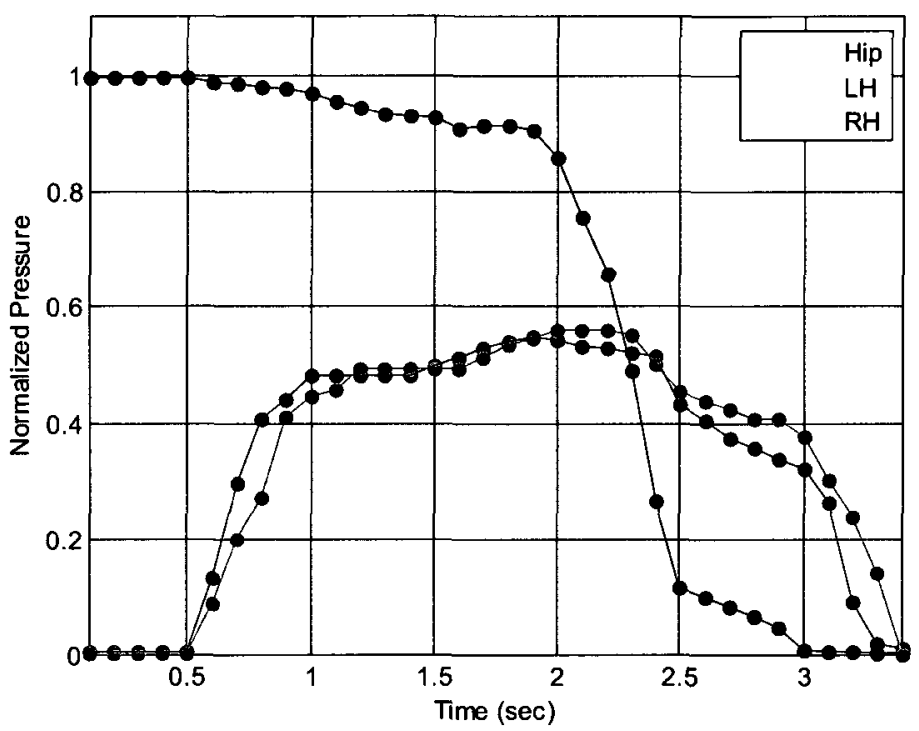

Figure 5.5: Ideal sit-to-stand transfer collected from control participant

The ideal sit-to-stand sequence result shown above identifies the hip, left hand, right hand regions using three curves. Red depicts the hip pressure and as seen from the graph initially the hip pressure is greatest while the hand pressures, blue (left hand) and green (right hand) curves are zero. This is a result of the sitting quietly phase which lasts approximately 0.5 seconds. During the next 1.0 second time period $(0.5 \mathrm{sec}-1.5 \mathrm{sec})$ there is a gradual increase in the hand pressure and a slight decrease in the hip pressure. This is the result of the subject placing their hands on the bed and beginning to transfer some of the hip pressure to the hands. The hand pressure continues to increase and the hip pressure begins to rapidly decrease during the transition period where the subject is lifting their hip region off of the bed and placing all of their weight in their hands ( $1.5 \mathrm{sec}$ $-2.5 \mathrm{sec}$ ). Once the hip pressure decreases to zero the hand pressure begins to decrease to zero therefore signalling the end of bed transfer. 


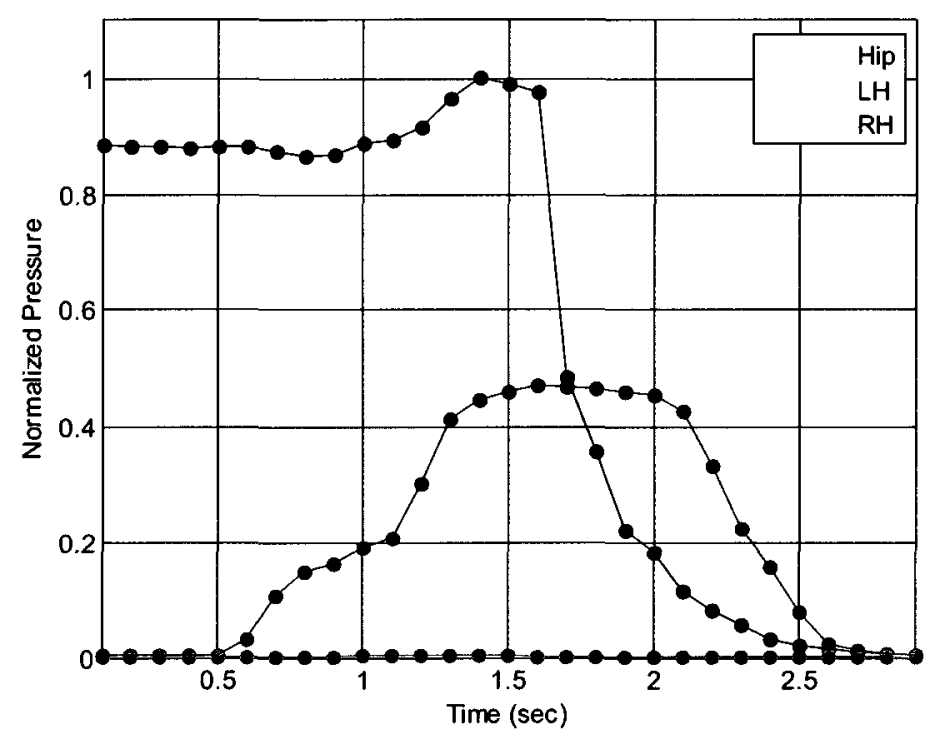

Figure 5.6: Sit-to-stand transfer using the left hand only (control participant)

The sit-to-stand sequence shown in Figure 5.6 above depicts a sit-to-stand transfer where only the left hand is used. The hip pressure curve is very similar to that of the ideal sit-to-stand sequence with one difference which takes place during the $1 \mathrm{sec}-1.6 \mathrm{sec}$ period. To start the subject is sitting quietly but this time they are not applying the maximum pressure during this period. The maximum pressure is actually applied prior to the hip lift off phase as a result of only the left hand being used during the transfer. Instead of the subject transferring their hip weight to both hands in the above scenario this pressure is only being transferred to the left hand and as a result the subject applies a larger force down into the bed with their hips. As the hip pressure begins to decrease (1.6 sec $-2.1 \mathrm{sec})$ the left hand pressure follows and the end of the sit-to-stand is completed once both pressures decrease to zero. Figure 5.7 below shows a sample result for when the sit-to-stand transfer involves only the right hand where the entire hip to hand pressure curve trend described in the left hand case equally applies. 


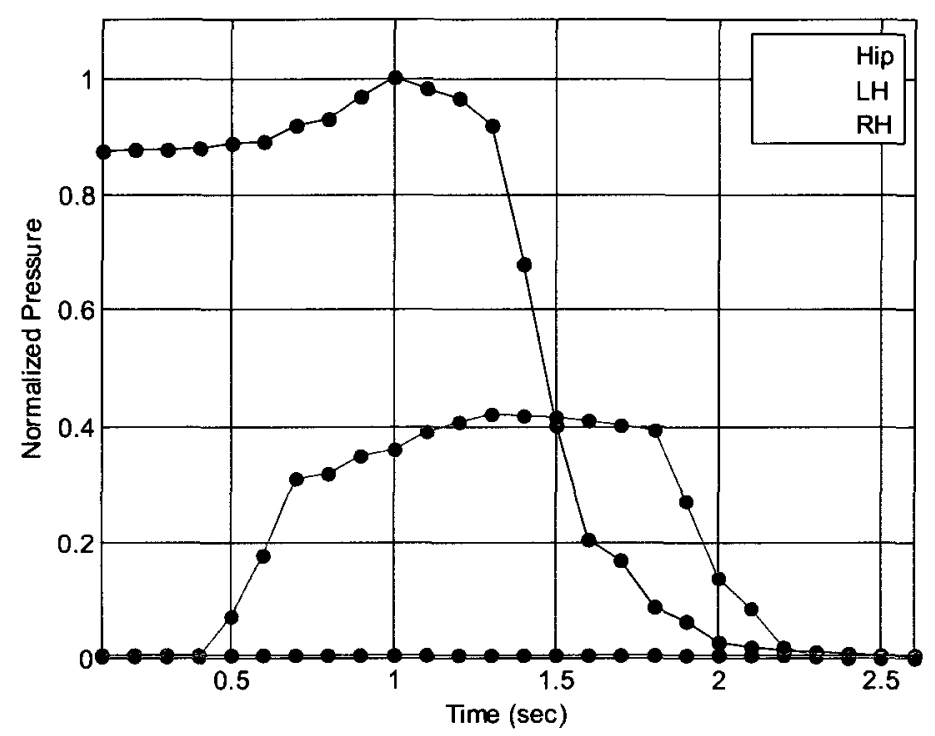

Figure 5.7: Sit-to-stand transfer using the right hand only (control participant)

From the results depicted by Figure 5.5 - Figure 5.7 it can be seen that this algorithm works very well. Each plot displays hip and hand pressure curves as expected based on the sequence type: ideal sit-to-stand, left hand only sit-to-stand and right hand only sit-tostand. This algorithm is very accurate when the initial assumption is correct; the subject is always sitting with their hips in the middle region of the bed and if they use their hands then the left hand will be used in the far region at the head of the bed and the right hand in the far region at the foot of the bed. The main disadvantage of this algorithm is that it depends entirely on the subject applying pressure in the three set regions described above. However these regions are not marked on the bed and the subject is not told where to place their hips or hands. Therefore if the subject places their hips or hands outside one of these boundaries during their sit-to-stand sequence, the algorithm will produce inaccurate results. 


\subsubsection{Binary Image Cluster Detection}

This region detection algorithm is a two step process based on iteratively searching a binary image for connected components. The first step in this region of interest algorithm is to convert the greyscale image to binary. This process is done by choosing a threshold value between the range of zero and one, zero representing black pixels and one representing white pixels. In performing the conversion each pixel in the image is processed by assigning a zero value if the pixel falls below the threshold and a value of one if the pixel is above the threshold value [63]. Figure 5.8 (a) and (b) are examples of a greyscale and binary image representing the pressure image generated during quietly sitting.

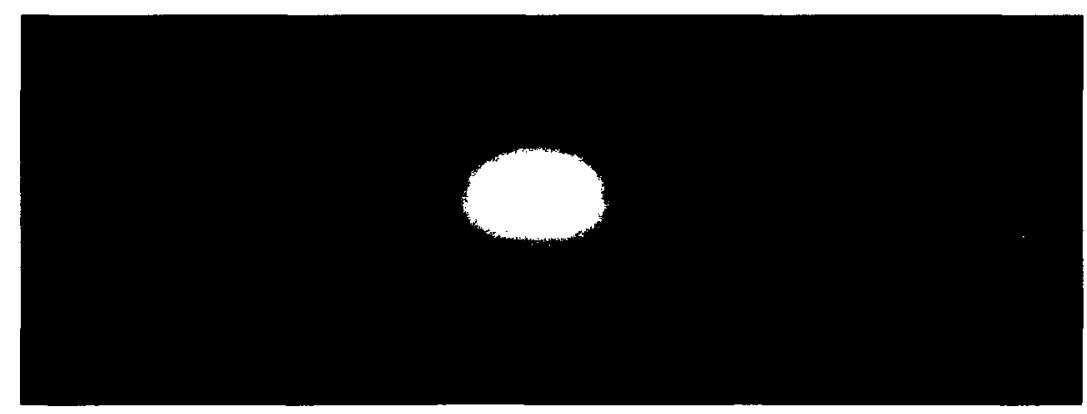

(a) - Greyscale image of the hip pressure

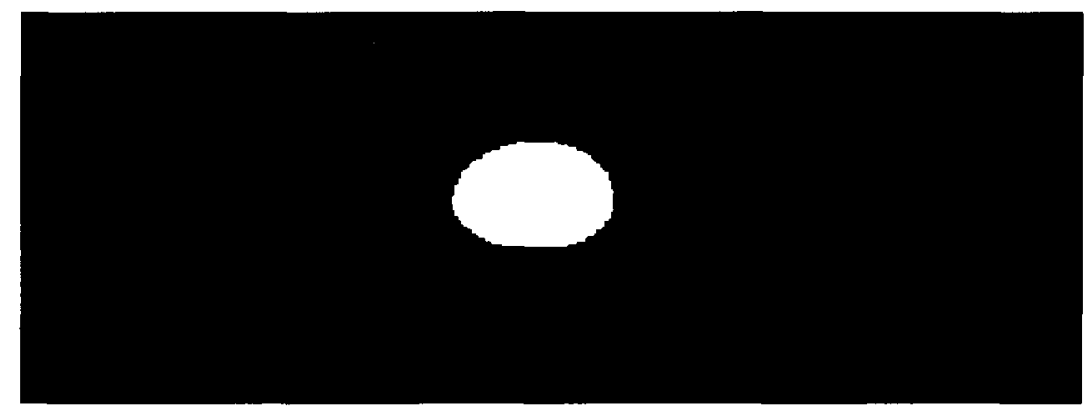

(b) - Binary image of the hip pressure

Figure 5.8: Sample greyscale to binary image conversion

Choosing the correct threshold value is a very important component of this algorithm. A low value will make it difficult to separate the hip and hand regions but a high value 
will cause low pressure regions to be undetected. In the case of the control sit-to-stand transfer a threshold value of $0.7(70 \%)$ was found to be ideal. Figure 5.9 shows the result for four different threshold values for (a) a hip pressure image and (b) a hip and hands pressure image.

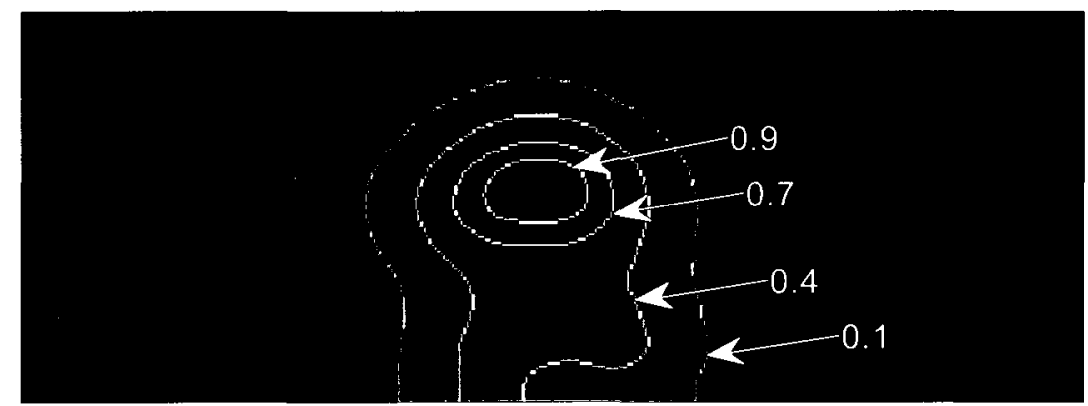

(a) - Hip pressure regions

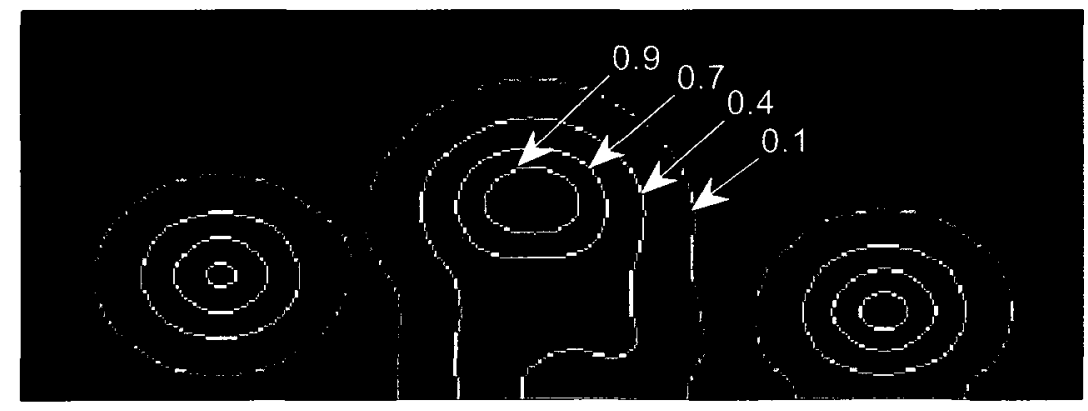

(b) Hip and hand pressure regions

Figure 5.9: Perimeter results for four different threshold values

The second step of the algorithm is called connected component labelling, which searches the binary image to find clusters of pixels based on pixel connectivity. In this case it is searching for a group of pixels that are 'on'; these are the pixels that have been assigned a value of one during the segmentation phase where we threshold the image. Each binary image is scanned from top to bottom and left to right using a measure of connectivity to group pixels and create an output label matrix with the same size as the image. A measure of 8-conductivity is used which means that pixels are connected if their edges or corners are touching, therefore if any two adjoining pixels are on; they are 
part of the same object. The algorithm moves along a row stopping at each on pixel, at this point it checks the surrounding pixels which have already been encountered and labels the current pixel accordingly. There are three possible cases that require checking. Case I, if none of the encountered neighbouring pixels have been assigned a value then the current pixel is assigned a new value in the label matrix. Case II, if one of the neighbourhood pixels has been assigned a label value then the current pixel is assigned the equal value in the label matrix. Case III, if more than one of the neighbours has been assigned a label then the pixel is randomly assigned one of the label values and a note is stored about the equivalence of these two labels. Once the label matrix has been completed the algorithm performs equivalence class resolution where it processes the label matrix and assigns a unique label to each cluster. Each pixel in the label matrix is replaced by the lowest label in its neighbourhood. Once the algorithm is complete the label matrix is made up of detected clusters each labelled with a different number, these clusters can be distinguished by assigning specific colors or labels [56]. In the case of hip and hand detection the labelled regions are used as masks for extracting specific areas from the original greyscale pressure images. The result for this approach applied to a sequence of three sit-to-stand images is shown in Figure 5.10.

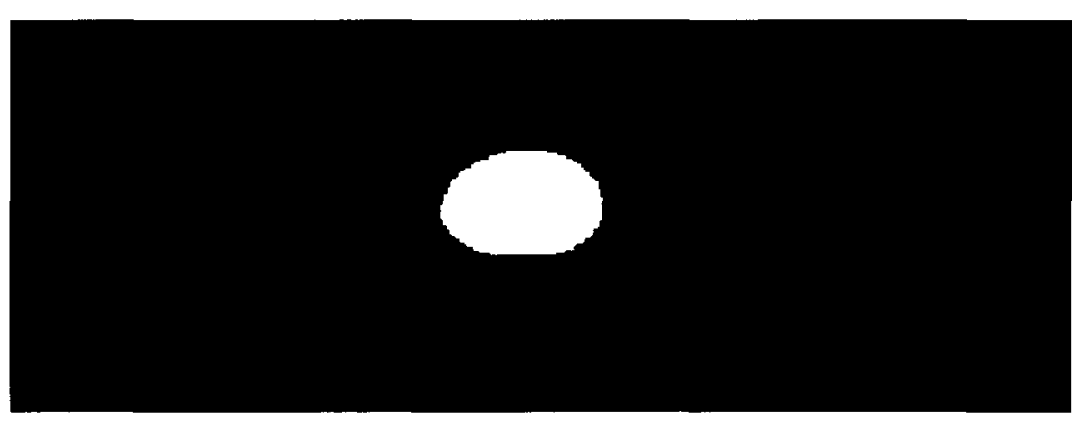

(a) - One region detected 


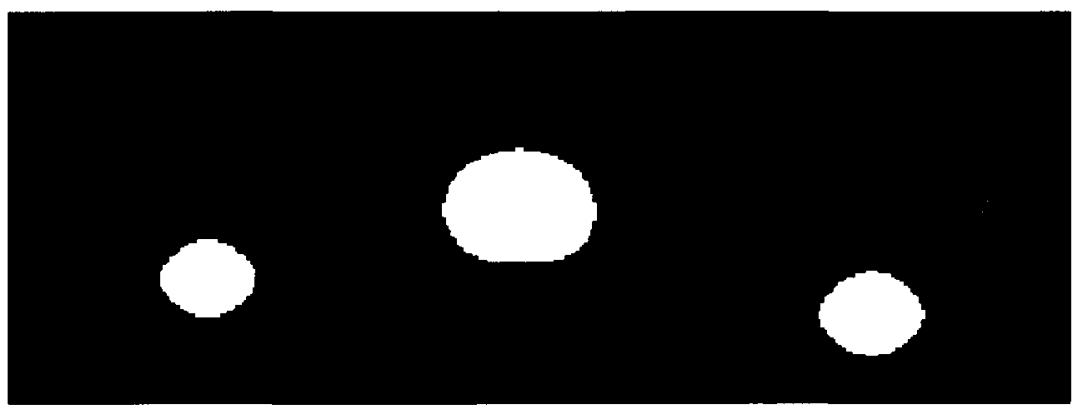

(b) - Three regions detected

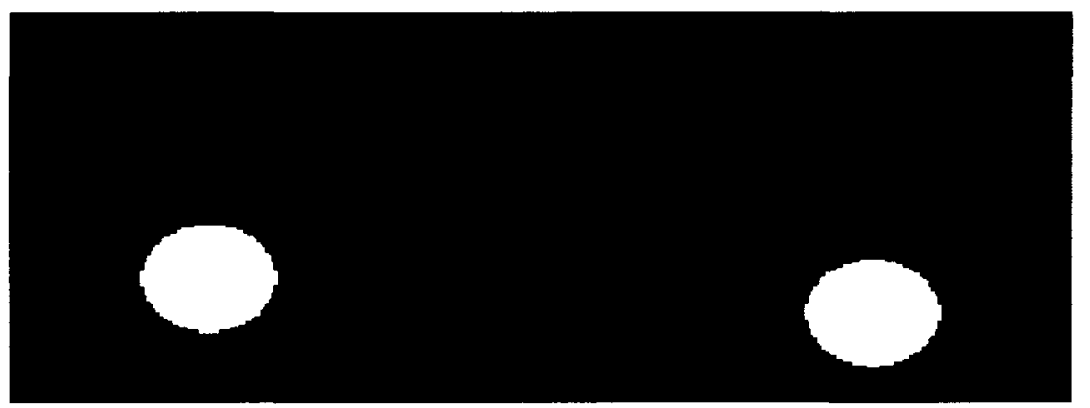

(c) - Two regions detected

Figure 5.10: Region detection results for a three image sequence

Another way to use the component labelling results is to identify only the perimeter of each region and to display this overtop of the original greyscale images. This result is shown in Figure 5.11 and is useful for visualizing which areas of the original image were identified as equivalent clusters.

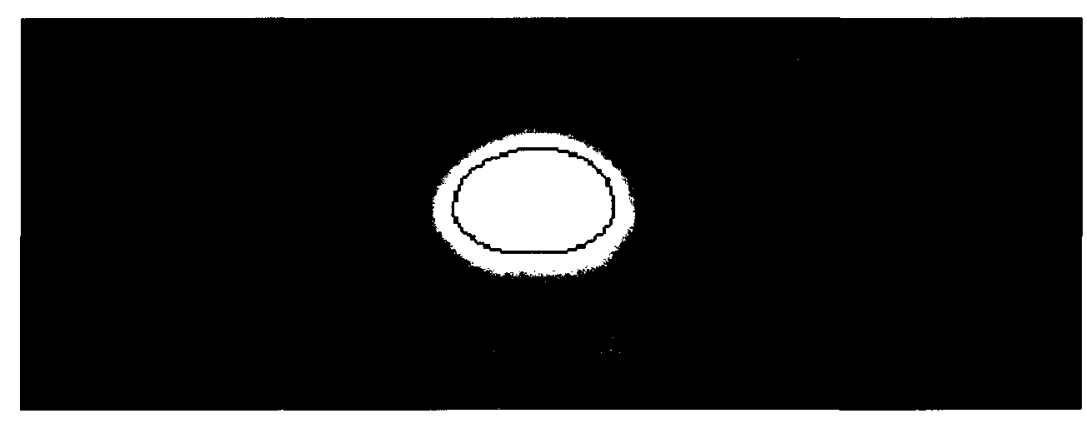

(a) - Displaying the perimeter from one detected region 


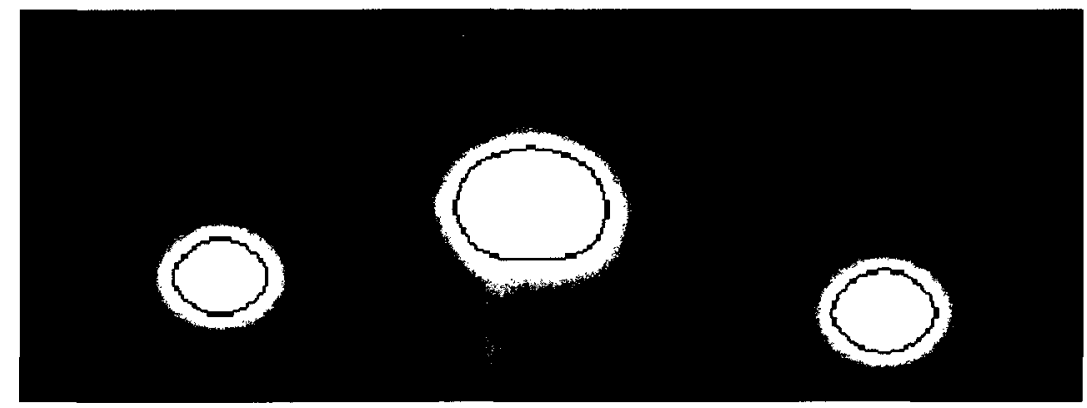

(b) - Displaying the perimeter from three detected region

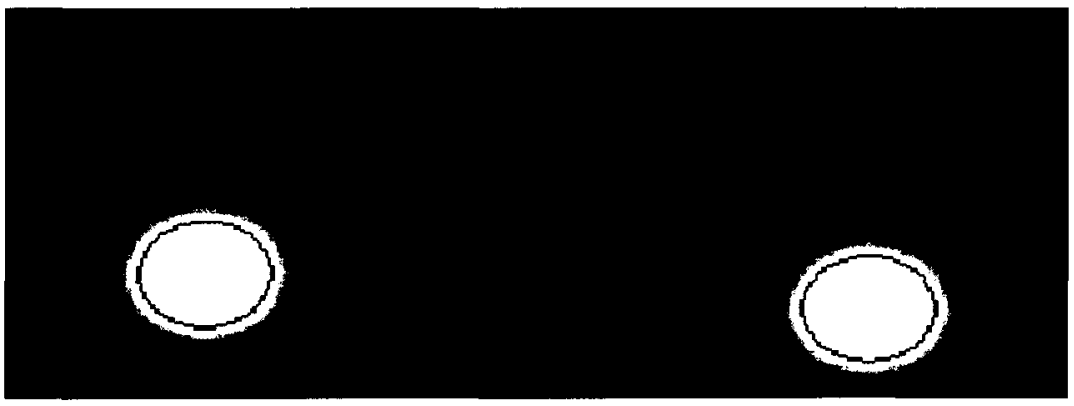

(c) - Displaying the perimeter from two detected region

Figure 5.11: Perimeter results for a three image sequence

The final result shown in Figure 5.12 displays each detected region with the corresponding label. The labels assigned to each region correspond to the order in which they are detected. Since the images are processed left to right a small label value corresponds to the bottom (right hand region) of the bed and a larger one corresponds to the top (left hand region) of the bed. In the case of sit-to-stand sequence images there are three possible labels: left hand, hip, right hand and these depend on the number of regions detected in the image. Case I, if one region is detected it corresponds to the hip. Case II, if two regions are detected they correspond to the left and right hand. Case III, if three regions are detected they correspond to the right hand, hip, and left hand. 


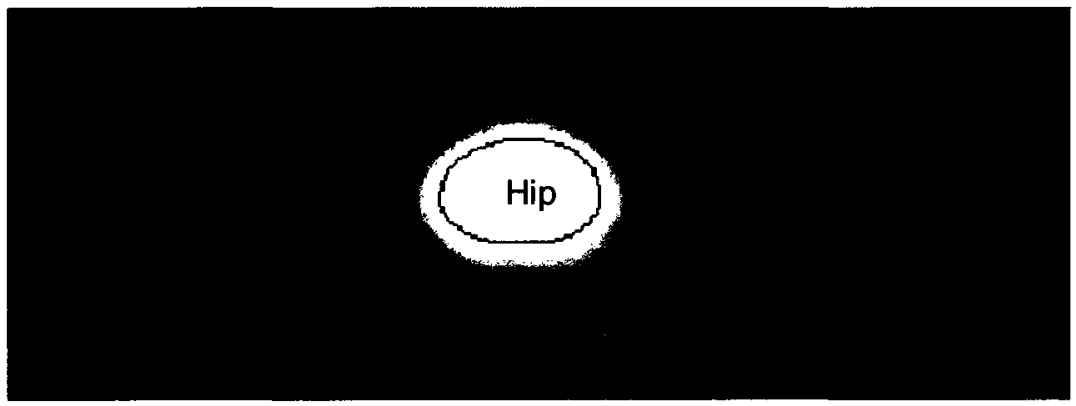

(a) - Detected hip region

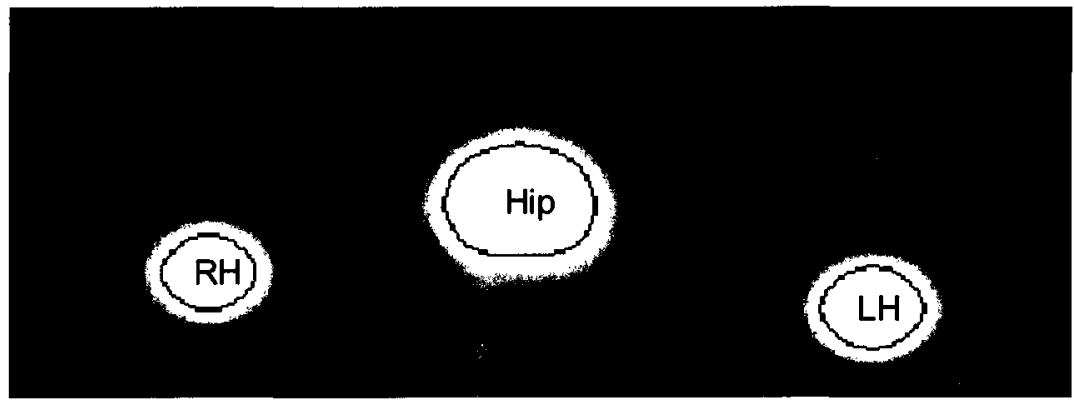

(b) - Detected right hand, hip and left hand regions

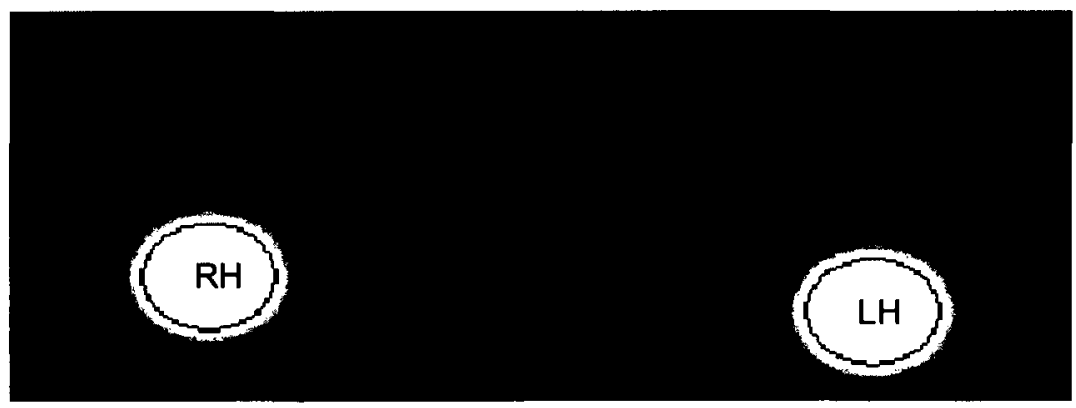

(c) - Detected right hand and left hand regions

Figure 5.12: Labelled detected regions results for a three image sequence

From the results it shows that this algorithm works extremely well for extracting hip and hand regions from images during the ideal sit-to-stand sequence but depends greatly on hand placement. If the subject places their hands too close to the hips, on top of the hips, or underneath the hips the algorithm will detect only one region and therefore will assume that no hands are being used. An example of this type of problem is shown in Figure 5.13. 


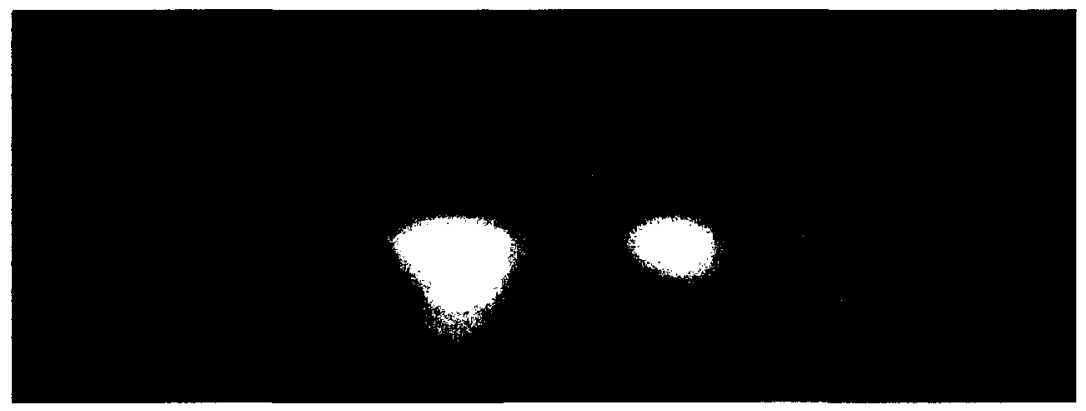

Figure 5.13: Detected region when hands are placed too close to the hips

The figure above is a sample taken during the sit-to-stand sequence when the subject is using both hands to lift off from the bed. The hand placement is too close to the hips therefore the entire region is detected as one and thus would be labelled as a hip according to Case I listed above. Another problem that could be encountered would result from the subject using only one hand when performing the sit-to-stand sequence. An image displaying this scenario is shown in Figure 5.14.

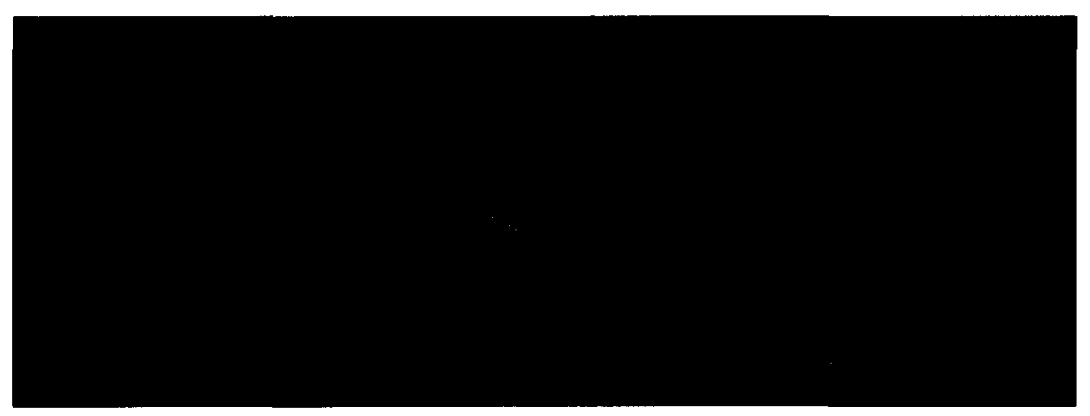

Figure 5.14: Detected region when subject is only using their left hand and it is placed close to the hips.

\subsubsection{Template Matching}

This region detection algorithm uses the Fourier transform to perform correlation between an image and a mask to locate features within an image. The mask represents the shape that we are looking to detect in each image, for the purpose of sit-to-stand analysis an object resembling the hips or hands will be selected. The correlation between the mask and image is calculated by using the fast convolution technique. Rotating the mask image 
by $180^{\circ}$ and then computing the fast Fourier transform results in a correlation image in which bright areas correspond to detected mask regions in the original image. These regions are extracted by finding the maximum pixel value in the correlation image and applying a threshold that is less than this maximum [63]. In the case of hip and hand region detection a threshold value of $0.85 *$ the maximum pixel value is used. For this algorithm the threshold value depends on how closely the mask image matches the image being processed and therefore a threshold of $85 \%$ was found to be the optimal value when performing hip and hand region detection. Figure 5.15 - Figure 5.19 shows five different masks and their corresponding results when applying this algorithm to a hip only image and a hip and hands image.

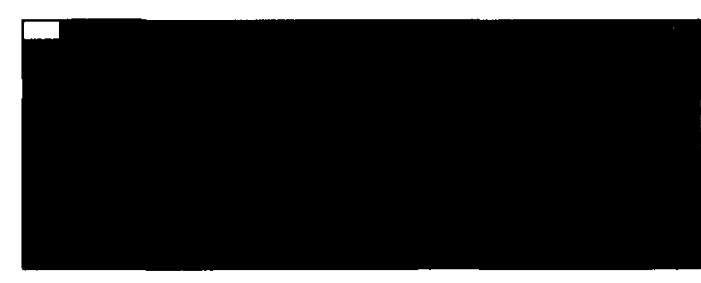

(a) Rectangle mask image

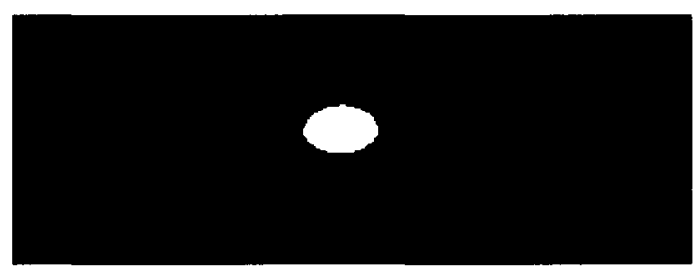

(b) Hip region detected

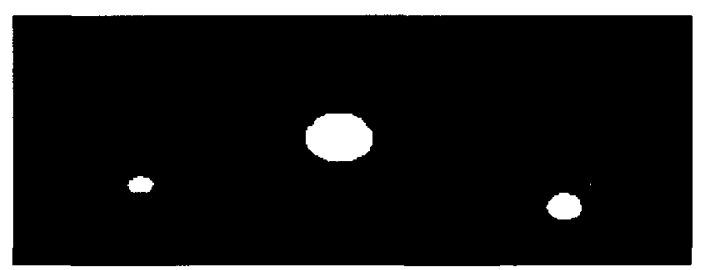

(c) Hip and hand regions detected

Figure 5.15: Template matching using rectangle mask image

The results for the rectangular mask used in Figure 5.15 are very good, the regions of interest are detected correctly as shown by the hip regions in part (b) and the hips and hands in part (c). The rectangular mask does not represent the shape of features we are expecting to see in the image and therefore a mask with a more rounded shape is selected 
next. Figure 5.16 - Figure 5.18 show results for three different masks, each of which has been created to resemble the hip regions.

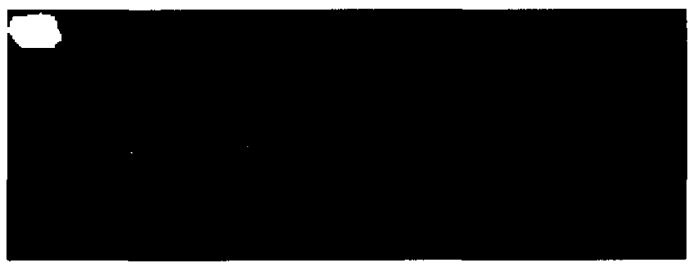

(a) Hip mask image

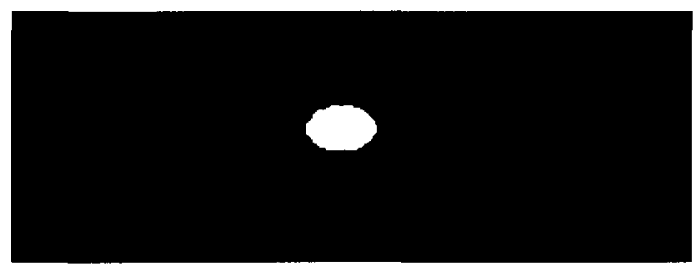

(b) Hip region detected

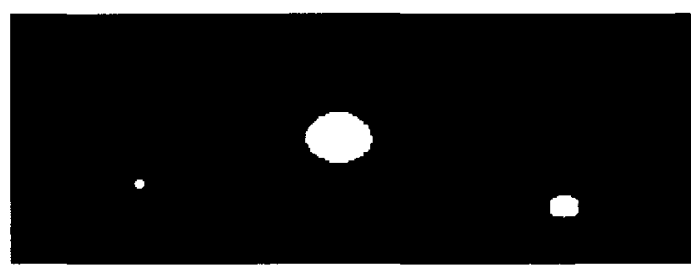

(c) Hip and hand regions detected

Figure 5.16: Template matching using sample hip mask image

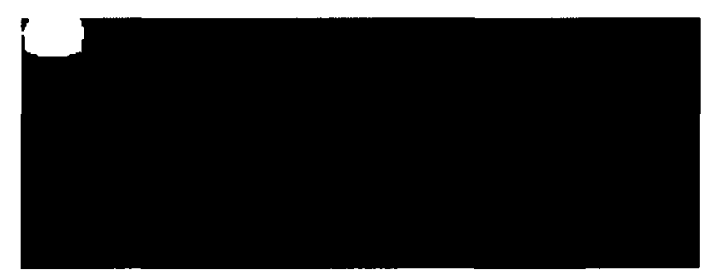

(a) Larger hip mask image

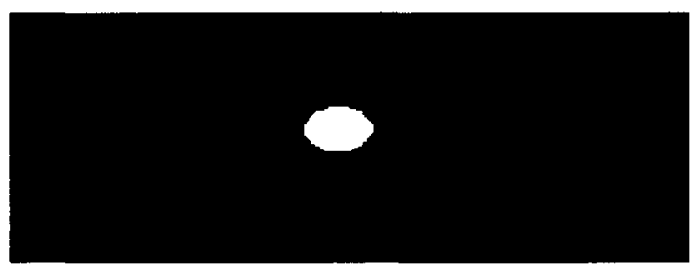

(b) Hip region detected

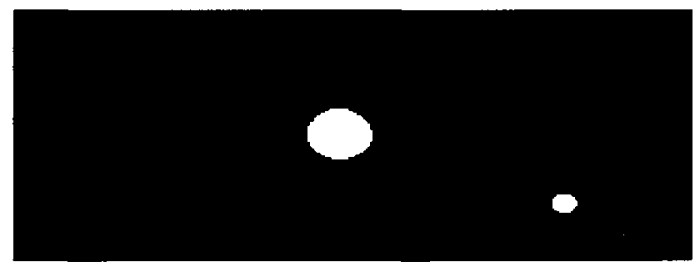

(c) Hip and one hand regions detected Figure 5.17: Template matching using larger sample hip mask image 


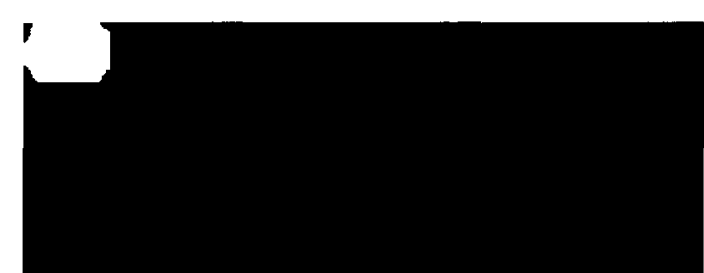

(a) Largest hip mask image

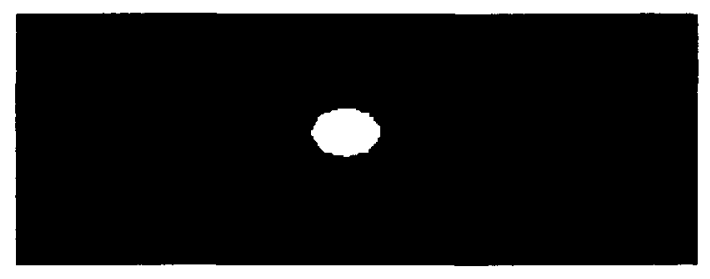

(b) Hip region detected

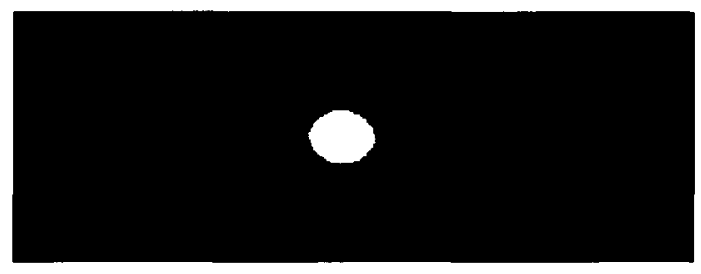

(c) Only hip region detected

Figure 5.18: Template matching using largest sample hip mask image

From the three sets of results above it can be seen that as the mask size increases the detection accuracy decreases. The hip regions are detected correctly in all 3 cases however the hand regions are detected as very small in Figure 5.16 part (c), only the left hand is detected in Figure 5.17 (c) and no hands are detected in Figure 5.18 (c). These results show that the mask size is very important when performing template matching. The masks in the above three figures are too big compared to the hand regions they are trying to detect. To help show the mask size dependency, the algorithm is run using a smaller square mask and the results are shown in Figure 5.19. 


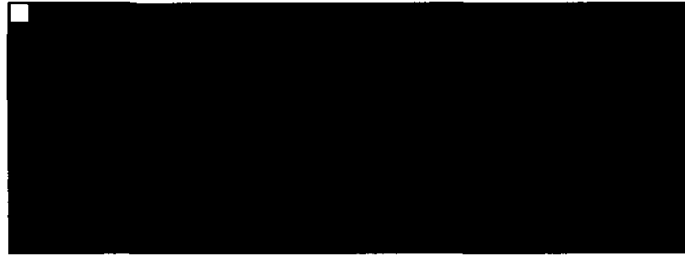

(a) Square mask image

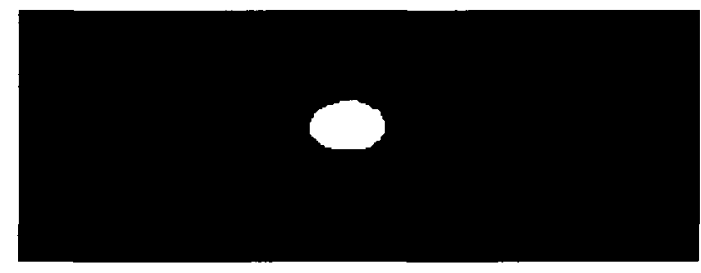

(b) Hip region detected

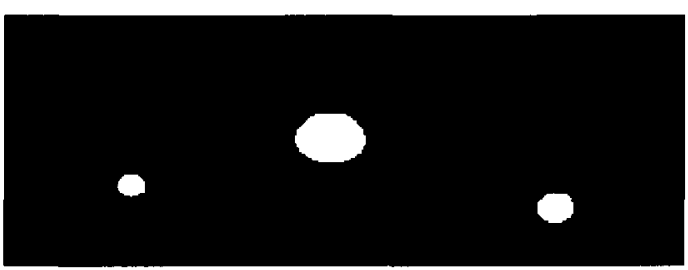

(c) Hip and hand regions detected

Figure 5.19: Template matching using square mask image

From the results above it can be seen that the smaller mask is able to detect the hip and hand regions extremely well. Overall this algorithm can yield very good results depending on the mask that is used. However as seen from the above results this can be a major disadvantage because the size of the results can vary greatly when using different masks, even ones that vary only slightly in size. Although the above results look good this algorithm wouldn't be ideal for clinical patient studies where the hip and hand region sizes vary greatly due to participant weight differences.

\subsubsection{Edge Detection}

Edge detection is a fundamental image analysis operation and this section looks at detecting regions of interest by using several different edge detection methods. Five different edge detection methods are described and tested on sample pressure images; their results are shown in Figure 5.20. When using edge detection to locate regions in pressure images, we are looking to distinguish regions where there is an abrupt change in pixel intensity by locating the set of pixels that are located along this boundary. Based on 
their underlying detection principles, edge detection algorithms can be grouped into three categories: gradient operators, zero-crossing operators and canny edge operators [64].

The gradient based method assumes that edges are made up of pixels with a high gradient when a specific gradient operator is applied. Examples of gradient based algorithms are: Prewitt filter, Roberts filter and Sobel filter, each applying a gradient vector differing in direction angle and magnitude. A disadvantage of using a gradient based edge detection algorithm is that they are very sensitive to noise. The derivative operator acts as a high-pass filter resulting in both false positive and false negative edge detection errors. False positives are pixels that are defined as edge pixels which are actually non-edge pixels and false negatives are the reverse [65].

The Laplacian of Gaussian method was the zero-crossing of the second-derivative type algorithm tested. This method starts by filtering the image with a smoothing Gaussian filter to reduce noise and then computes the Laplacian zero-crossings to locate regions of rapid intensity change, also known as edges [65].

The Canny method uses both the first derivative and second derivative concepts. The algorithm is based on smoothing the image using a Gaussian filter and then calculating the gradient of the image in order to find the edge strength. In order to detect linked edges non-maxima suppression is applied to the gradient magnitude and double thresholding is used to reduce error. The Canny method is considered to be the most accurate edge detection algorithm [66]. 


\section{$\infty$}

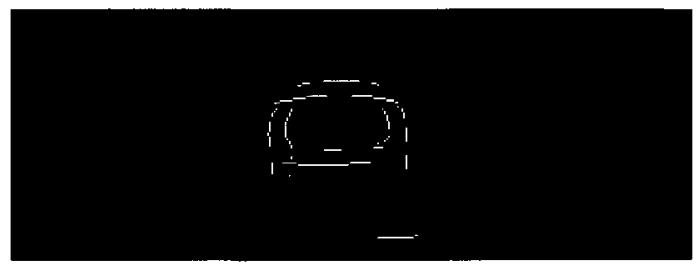

(a) Original Image

(b) Prewitt Method

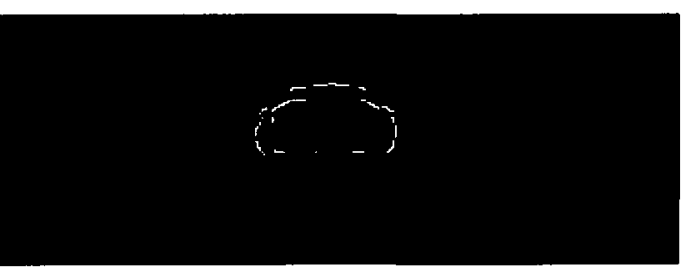

(c) Roberts Method

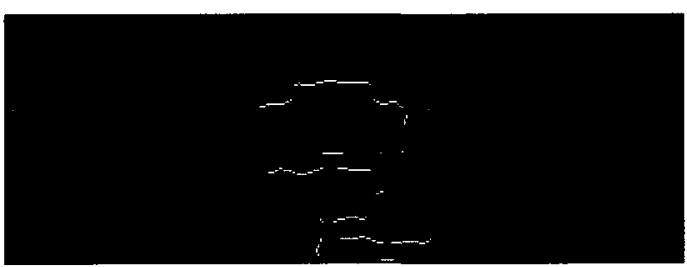

(e) Laplace of Gaussian (LoG) Method

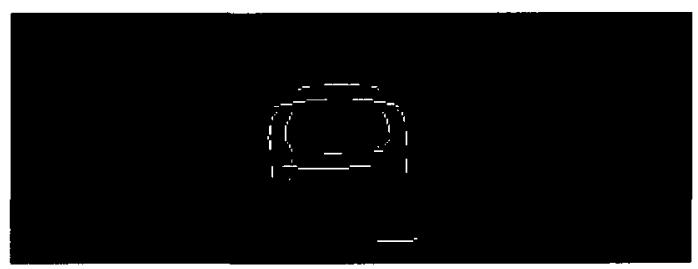

(d) Sobel Method

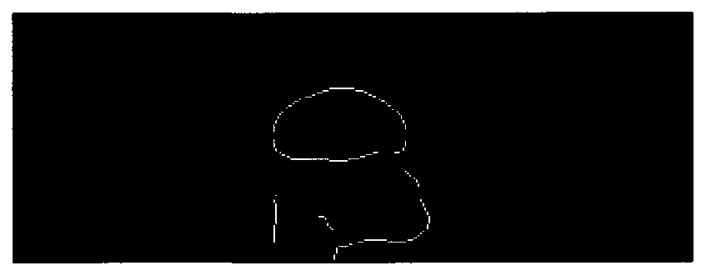

(f) Canny Method

Figure 5.20: Five edge detection algorithm results

From the results in Figure 5.20 it can be seen that the Laplace of Guassian Method and Sobel Method provide very poor results when applied to the hip pressure image in Figure 5.20 (a). A more complex hip and hand pressure image is processed by the superior three methods: Prewitt, Roberts and Canny, and the results are shown in Figure 5.21 for a different pressure image. 


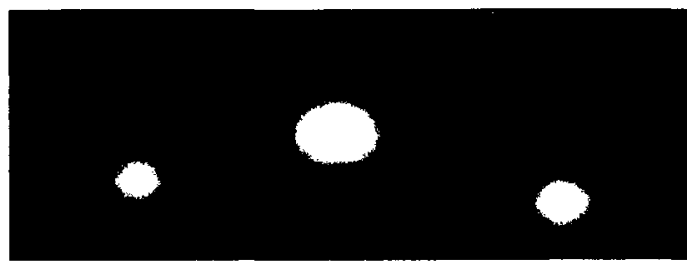

(a) Original Image

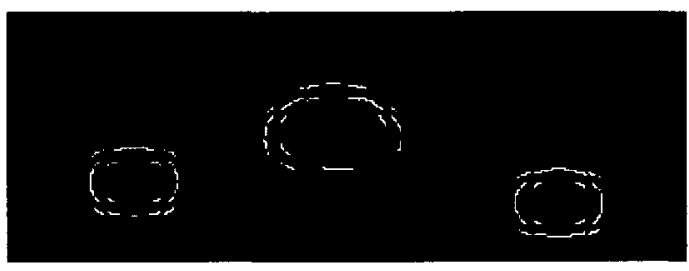

(c) Roberts Method

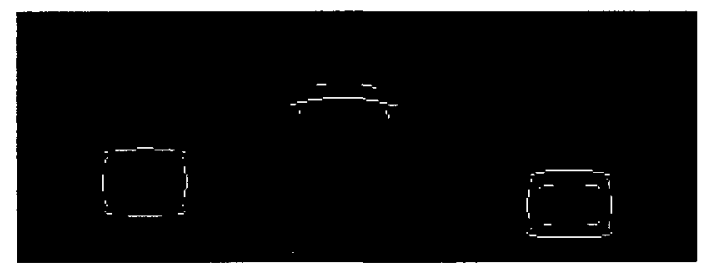

(b) Prewitt Method

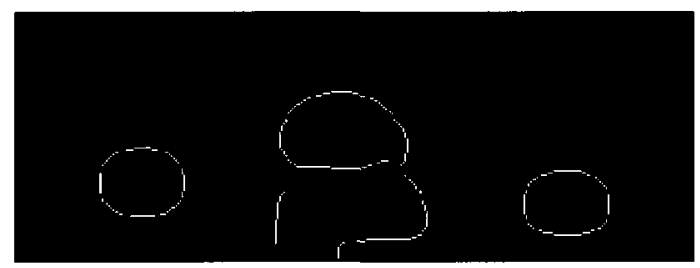

(d) Canny Method

Figure 5.21: Three edge detection algorithm results

When applying the above three edge detection methods to the hip and hand pressure image in Figure 5.21 (a) it can be seen that the Canny method performs the best by far. The result displayed in Figure 5.21 (d) is a detailed outline of the hips and hands extracted from the image with minimal noise. Figure 5.22 shows results for another pressure image where only the hand regions are being processed using the Canny method.

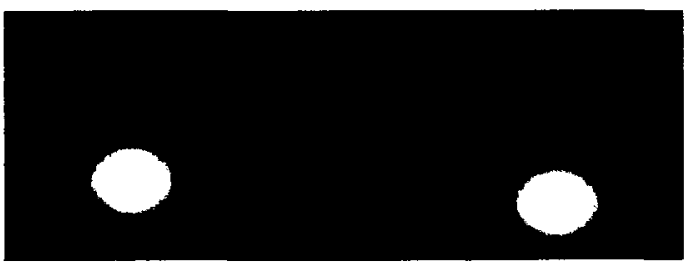

(a) Original Image

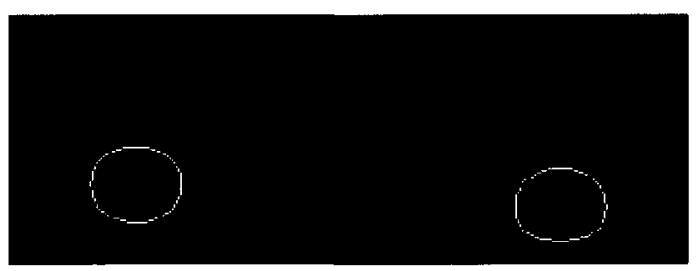

(b) Canny Method

Figure 5.22: Canny edge detection result

Although the Canny Method gives superior results when processing the three sample images shown, overall edge detection is not the ideal algorithm for region detection of pressure images. Edge detection is ideal for images that contain distinct lines and this is not the case when it comes to the pressure images generated from the mat data. As 
mentioned in Chapter 4 there is a step in the image creation process in which we blur the original data to overcome the spatial separation between pressure sensors. An example of this limitation is shown in Figure 5.23 where the Canny edge detection method is applied to an image representing a seated participant. The result showing the binary image cluster detection is also displayed for comparison purposes.

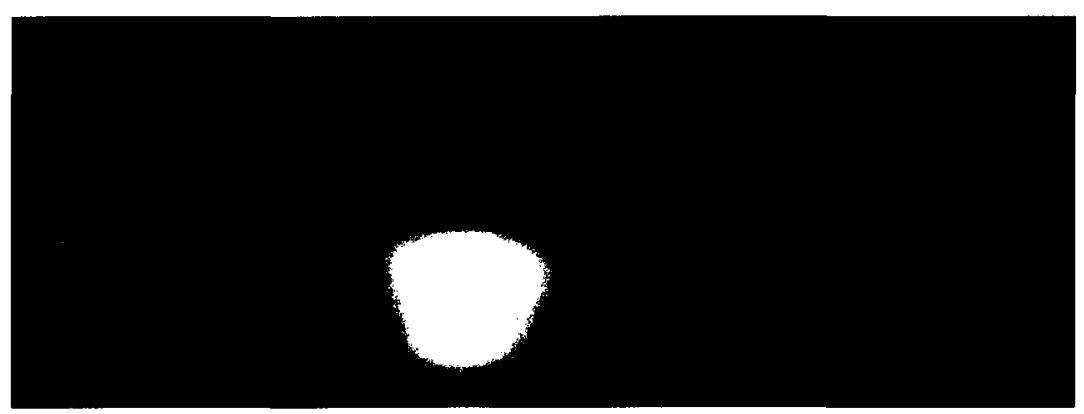

(a) - Original image

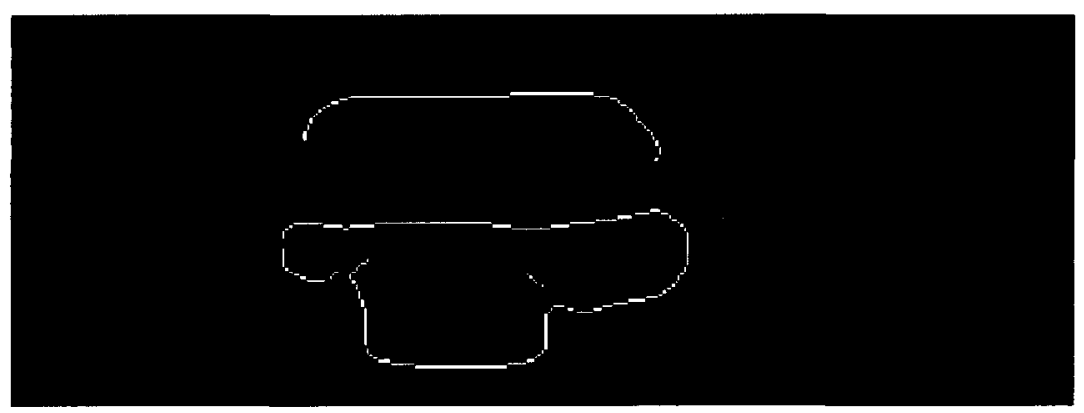

(b) - Canny edge detection result

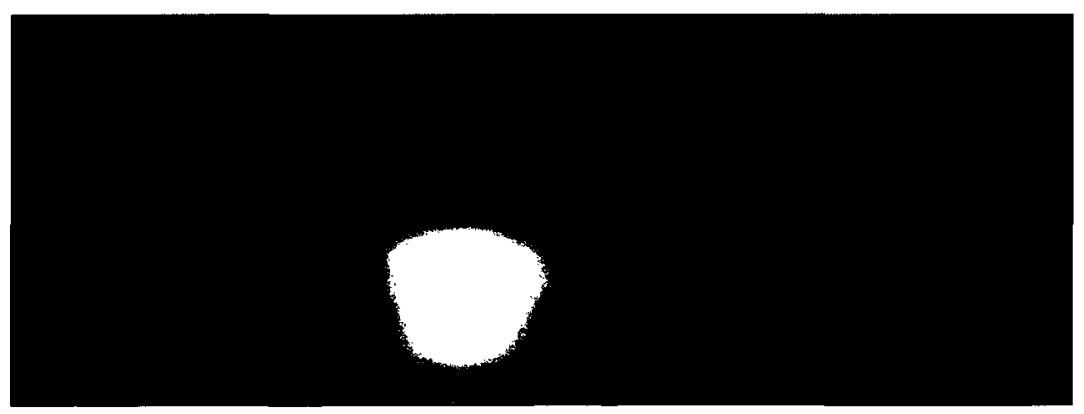

(c) - Binary image cluster detection result

Figure 5.23: Example displaying the main disadvantage of edge detection

The Canny edge detection method performed the best when it was applied to ideal sitto-stand image sequences. However from the results shown in the images displayed in 
Figure 5.23 it is clear that edge detection is not the best suited region of interest algorithm when applied to pressure images.

\subsection{Challenges and Limitations}

A common problem with the above three region detection algorithms is that they are all performed on a frame by frame basis and there is no information being carried from one frame to the next. This is a major limitation when processing images that are composed of various stages in the sit-to-stand transfer which is represented by a series of images. The various problems affecting the results can be divided into two categories, technological limitations and clinical challenges.

\subsubsection{Technological Limitations}

There are two technological limitations, resolution and sampling rate. The resolution for the bed mat was chosen based on the cost and resulting benefits associated with the equipment that was purchased from Tactex Controls Inc. Tests were conducted on a higher resolution floor mat for comparison purposes. The data collected from the floor mat was more detailed but placing this type of mat underneath a bed for the bed exit study was infeasible. This mat is only 24 inches by 24 inches and is made out of a very hard material which would cause the participants much discomfort when performing sitto-stand transfers. Therefore it was decided that the bed mat was the best choice for this study, even though using it would result in some problems. The separation between pressure sensors in the bed mat is several centimeters and this low resolution makes it difficult to detect the separation between the hips and hands in each frame. If the hand is only separated from the hips by one pressure sensor, then when the image is blurred to 
overcome the spatial resolution the hand and hip regions will be joined together to form one region.

The sampling rate is the second technological limitation, recording data at $10 \mathrm{~Hz}$. This rate was also chosen based on the cost involved with increasing the sampling rate and the benefits that would result from this increase. The long term goal of this project is to perform 24 hour bed transfer monitoring for subjects at home and therefore greatly increasing the sampling rate could cause data processing issues. The problem associated with the current sampling rate is that the sit-to-stand transfer can be completed very quickly therefore limiting the amount of information that is collected for a particular transfer.

In the future higher resolution and sampling rates may be more feasible therefore eliminating some of these problems.

\subsubsection{Clinical Challenges}

The main clinical limitations that would affect the algorithm results are hand placement and unexpected movement. Unusual placement of the hands would greatly affect the results because hands placed underneath or on top of the thighs would go undetected when analyzed using the algorithms presented in Section 5.3. Other scenarios that would cause problems would be the use of only one hand or subjects, who place both hands very close to their thighs during their sit-to-stand transfer. Clinical situations where the subject shuffles around the bed prior to standing or bounces themselves into a standing position would yield poor results from all three algorithms. An example could be if a participant uses their hand to lift up their hips but then places them back down in a slightly different location, therefore bouncing into a new starting position. Since the algorithms above label 
each frame based on only the regions detected in the current frame they would mislabel many of these frames, mistaking the hip region for the hand region and vice versa.

\subsection{Recommendation}

One suggestion for solving the challenges and limitations listed above is to look at the sitto-stand as a sequence of frames, therefore keeping track of the changes that occur between frames rather than processing each frame individually. This idea is based on keeping track of the increasing and decreasing pressure that is being applied by the subject. Implementing this idea involves calculating the difference between two frames in a sequence, separating the positive difference from the negative difference and amplifying both to increase their intensity and make them easier to visualize. The positive difference regions represent regions of increased pressure and the negative difference regions represent regions of decreased pressure. Figure 5.24 shows a sequence of two images and Figure 5.25 shows the corresponding increasing and decreasing pressure images.

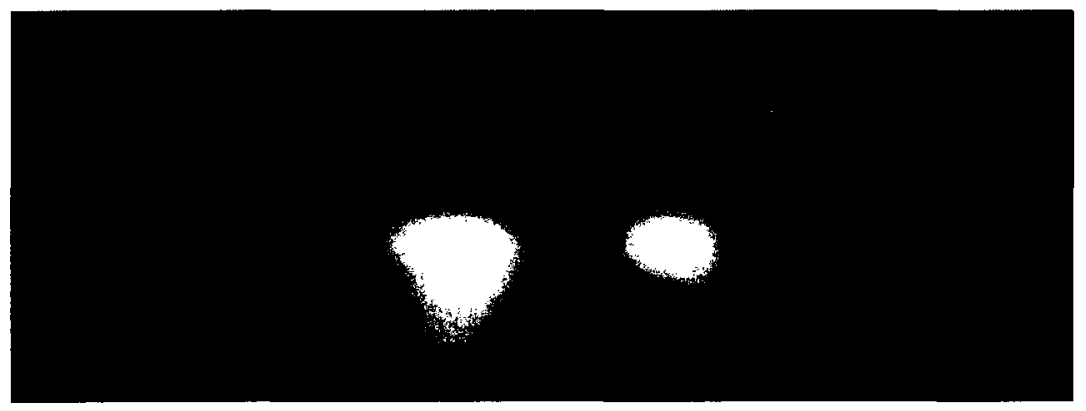

(a) Current Frame \#14 


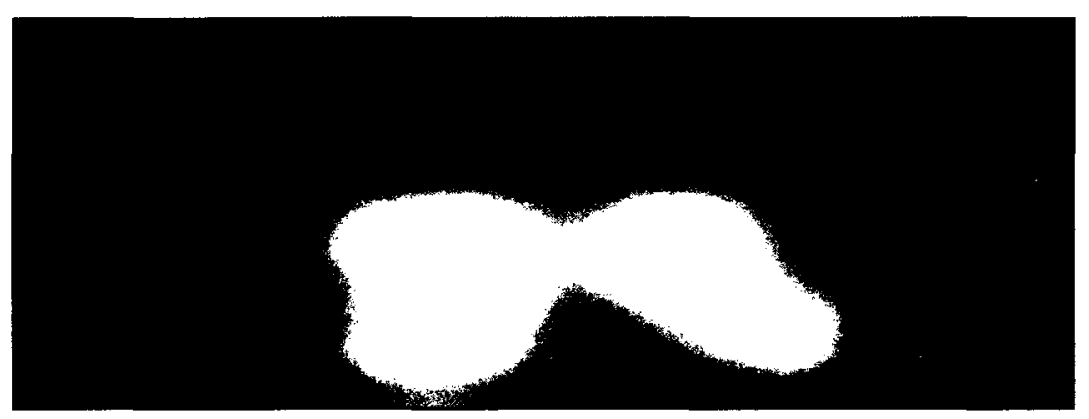

(b) Next Frame \#15

Figure 5.24: A sequence of two images extracted from a sit-to-stand sequence

When looking individually at the above two images it is difficult to see any difference between them. However when looking at the difference images between the two frames it becomes clear that there is a change; there is an increase in the pressure in both hand regions and a slight decrease in pressure in the hip region.

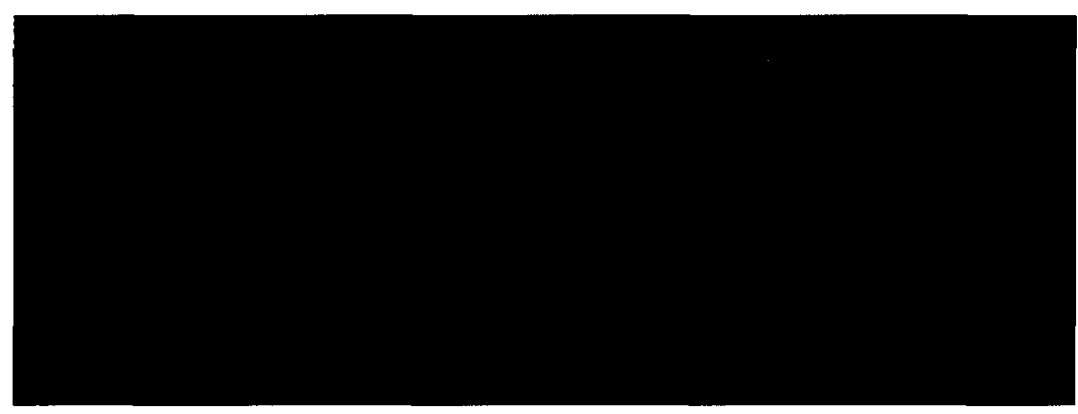

(a) - Decrease in Pressure (-ive pixels)

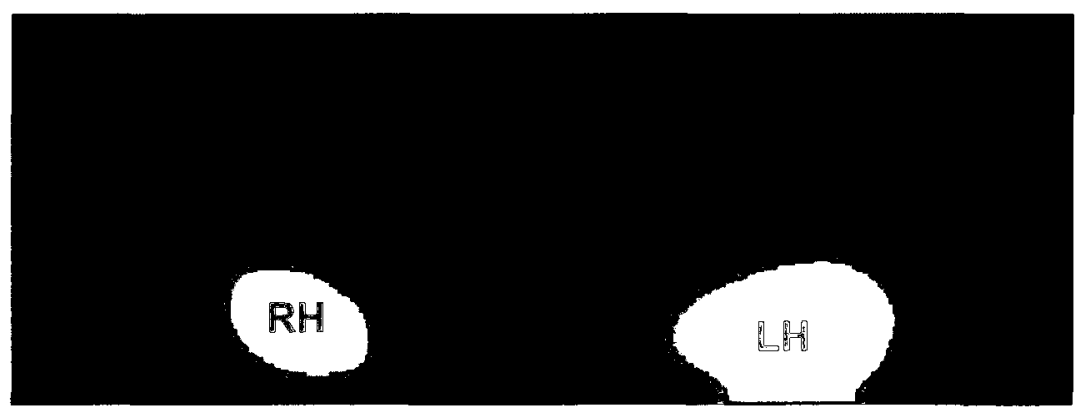

(b) - Increase in Pressure (+ive pixels)

Figure 5.25: Decreasing pressure and increasing pressure regions

Therefore examining the difference between frames can provide important additional information when processing sit-to-stand data from a non ideal case. The idea of tracking 
the increasing and decreasing pressure between frames can help eliminate some of the limitations and problems mentioned. Combining this information with the frame by frame algorithms presented would greatly increase the reliability and intelligence of the presented ROI algorithms. 


\section{Chapter 6:}

\section{Sit-to-stand Segmentation and Timing}

\subsection{Introduction}

The following diagram shows the thesis flowchart presented in Chapter 1 with the specific areas discussed in Chapter 6 outlined in red.

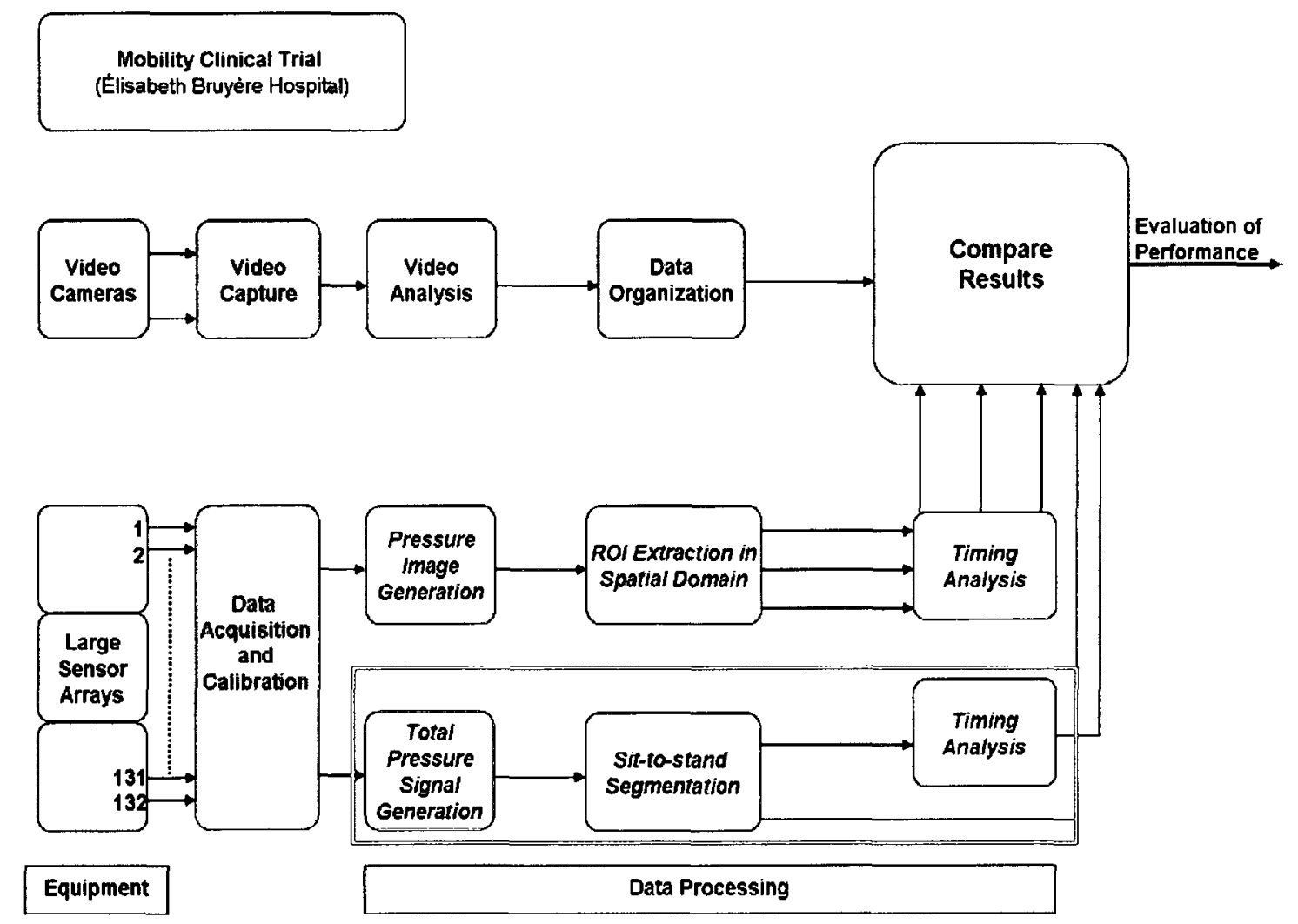

Figure 6.1: Thesis Flowchart 
A more detailed Chapter 6 flowchart is shown below.

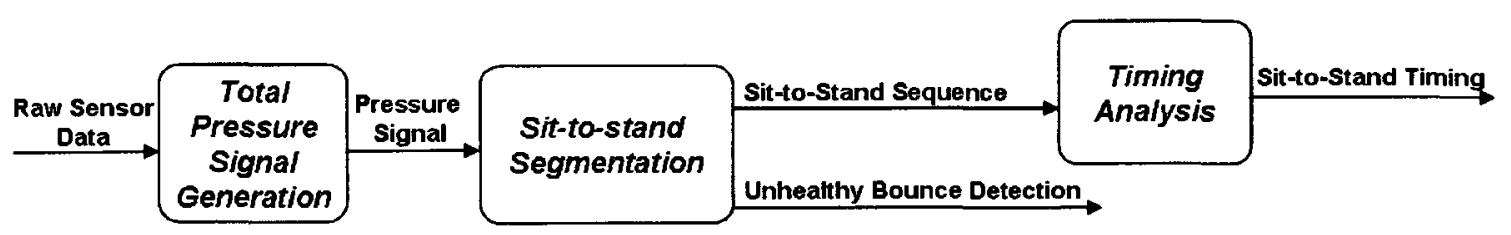

Figure 6.2: Sit-to-stand Segmentation and Timing Flowchart

The sit-to-stand transfer from a bed is almost universally the first task people in the western world perform after sleeping. Although seemingly a very simple task elderly people find it quite difficult to perform and therefore it can be used as a measure of health. The sit-to-stand transfer can be looked at as a movement cycle which has often been divided into several phases. Nuzik et al. [67] divides it into two phases: flexion and extension of the trunk. Schenkman et al. [68] and Riley at al. [69] divide the movement into four phases: flexion momentum, momentum transfer, extension and stabilization. Millington et al.[70] divides up the sit-to-stand cycle into three phases: weight shift, transition and lift. This chapter focuses on extracting these three phases using data collected only from the pressure mats located underneath the bed mattress. The three phases have been described by Millington et al. as a part of a study analyzing the difference between normal and abnormal sit-to-stand movements [70]. The first phase is the flexion phase of the trunk, during this phase the person begins to shift their posture forward therefore pushing their hips into the bed. The second phase is the transition phase which starts with the person leaning their trunk forward even further and ends once the person has started to slowly lift their weight off of the bed. The third phase is the lift phase which starts once the person rapidly starts to lift their weight off of the bed and ends once the person's buttocks are entirely off of the bed. 


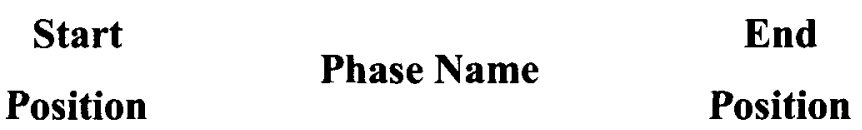

Phase I
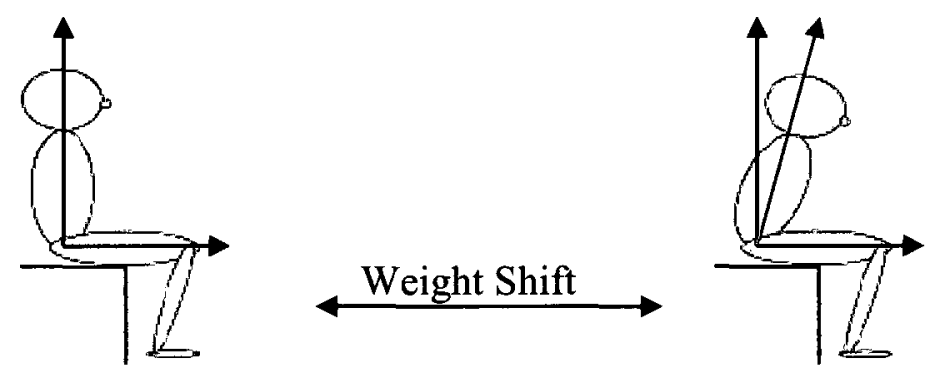

Phase II
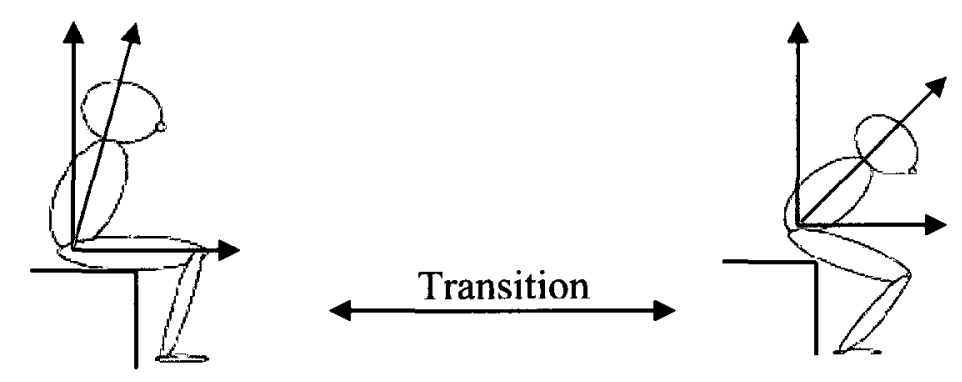

Phase III
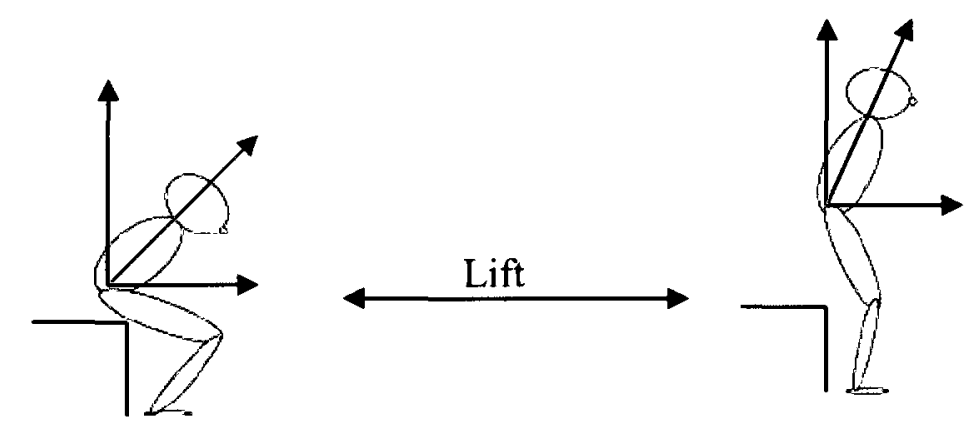

Figure 6.3: Sit-to-stand phases [71]

In the past these phases have been identified by looking at video footage of participants performing the sit-to-stand sequence or by analyzing collected data from various devices (ex. accelerometers [16], gyroscopes [18], and dynamometer [71]) which are attached to the participant during the experiment. This chapter focuses on extracting phase timing information from each sit-to-stand transfer performed by each of the 25 participants during the clinical trial. The key difference between this study and previous 
ones is the use of pressure sensitive mats, a non-invasive technology, to collect and analyse data from participants while they perform sit-to-stand transfers from a bed.

The ideal sit-to-stand sequence with Phase I, II and III identified is shown in Figure 6.4 .

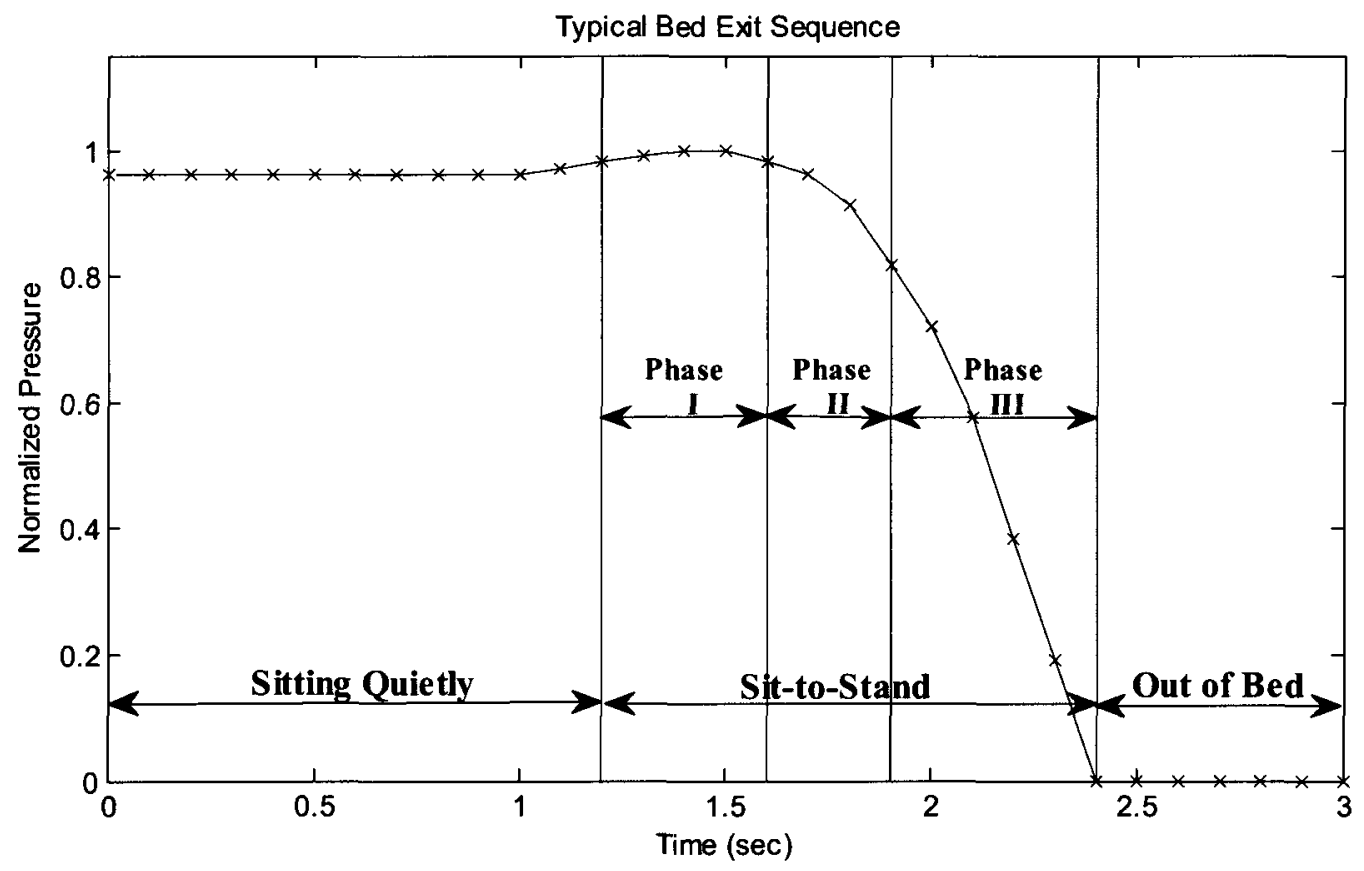

Figure 6.4: Simulated pressure curve showing an ideal sit-to-stand movement

The above curve represents the total pressure being applied to the bed during an entire sit-to-stand transfer time sequence. The pressure curve is generated by summing all of the taxels (pressure sensors) located underneath the bed mattress for the entire time sequence. This pressure curve is then normalized based on the highest pressure sum that is calculated during each sequence.

The curve shows a constant reading during the sitting quietly stage which happens at the beginning, prior to the sit-to-stand movement. The pressure curve is zero during the out of bed stage which happens once the sit-to-stand movement has been complete and there is no longer any contact between the subject and the bed. The three phases making 
up the sit-to-stand movement are also identified in the plot. Phase one shows a slight increase in pressure due to the subject pushing into the bed for an instant and then beginning to transfer their weight forward by leaning their trunk forward as seen in Figure 6.3. Phase two shows a slight decrease in the total pressure resulting from the subject transferring weight from the bed to the feet. Phase three shows a major decrease in the total pressure due to the subject completing the sequence by rapidly transferring the remainder of their weight from the bed onto their feet.

The pressure sensitive mat used to collect sit-to-stand data was located underneath the bed mattress during the clinical trials; therefore the total pressure data calculated from the mat is dependent on the subjects' weight. In order to make results from all of the different participants comparable, the results presented throughout the remainder of this chapter will be shown using normalized pressure for the sit-to-stand transfer time sequences. The participant weight dependency is shown in Figure 6.5. 


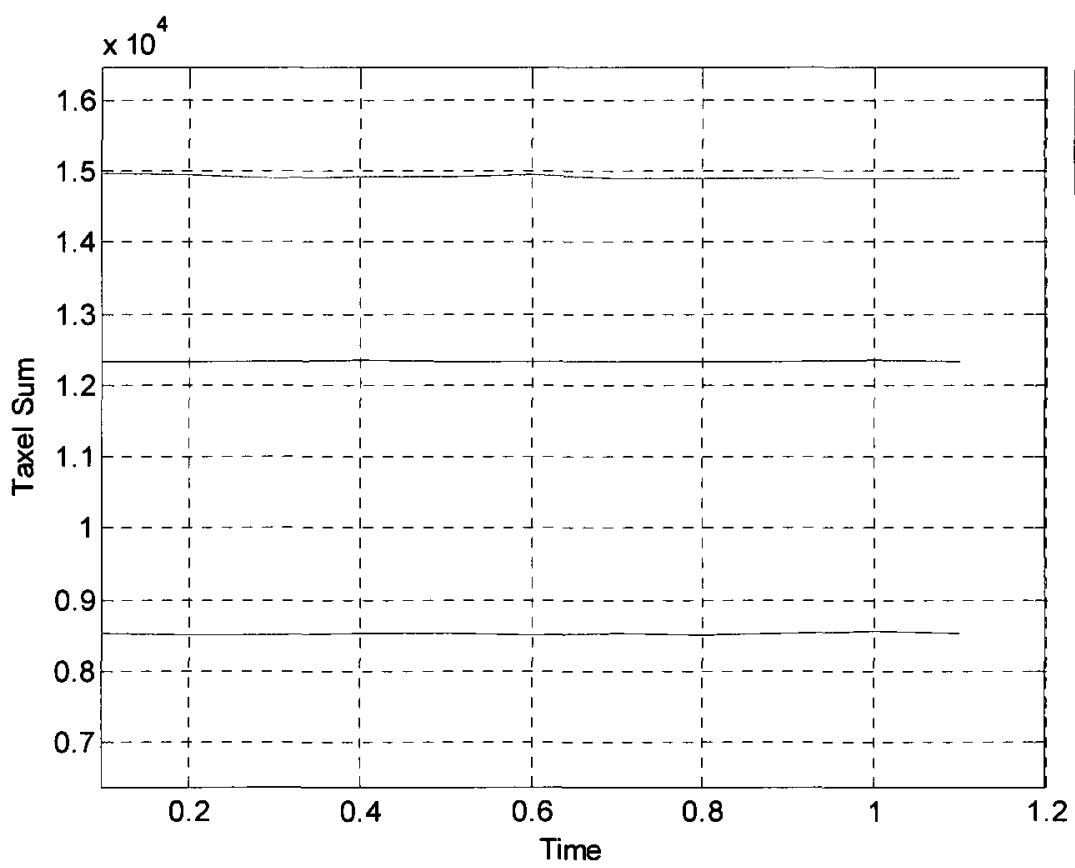

Participant $A=85 \mathrm{~kg}$

Participant $B=64 \mathrm{~kg}$

Participant $\mathrm{C}=44 \mathrm{~kg}$

Figure 6.5: Total pressure values for three participants with their corresponding weight

values

As seen from the plot above, the total pressure sum values can vary greatly depending on the weight of the participant. Throughout the remainder of this chapter, all results will be presented in Normalized Pressure units versus Time.

\subsection{Sit-to-stand Phase Detection Algorithm}

This chapter presents an automated algorithm which examines individual sit-to-stand sequences, identifying the start and end locations of the three phases described above. Each phase start time is marked using a colour coded marker and the length of each phase is calculated as the length of time between the start of the current phase and the start of the next phase. The end of the sit-to-stand transfer is also detected and marked with a red phase marker. The total sit-to-stand transfer time is calculated using this end marker as well as the start of phase one marker. 


\subsubsection{Algorithm Description}

The algorithm processes each sit-to-stand sequence by analyzing the normalized pressure curve from beginning to end. The algorithm assumes the first five frames to be resting or preparation frames and therefore averages these to calculate a resting baseline pressure. Next the algorithm searches along the signal for the phase one start time by comparing sequential frames until an increase of more than one percent between the current frame and previous frame is found, or an increase of more than one percent between the current frame pressure and baseline pressure is found. The slight increase in pressure marks the start of the weight shift phase in which the bed pressure is increased due to the subject shifting their weight forward and pushing their hips further into the bed. This increase in pressure was verified by examining corresponding foot pressure data which showed that during this instant the foot pressure decreases which results in an increase in the hip pressure being applied. This motion was also seen when watching video recordings of participants performing the sit-to-stand movement; most often the participants would lift up the entire toe region of their foot or sometimes even the entire foot when starting their sit-to-stand transfer. The weight shift phase lasts as long as there is a constant increase in pressure or until the pressure reaches a maximum and the start of phase two is detected.

Phase two is the transition phase, which represents the subject transitioning from transferring their weight forward to starting to decrease the pressure being applied to the bed. The beginning of phase two is detected by searching the sit-to-stand pressure signal for the last peak value greater than $85 \%$ of the baseline pressure value. The remainder of the transition phase is detected by processing the signal from the phase two start marker onwards. The algorithm sequentially compares consecutive pressure values searching for 
a decreasing pressure difference between one and five percent. Phase two is terminated once a decrease of greater than five percent is detected.

The start of phase three is detected once a decrease of more than five percent between consecutive pressure values is calculated. This signals the lift phase of the sit-to-stand cycle and this phase ends once the end of the cycle is reached and the subject is off of the bed. The end of phase three marker is used to calculate the total phase three and total sitto-stand times.

A plot of the sit-to-stand transfer performed by an older healthy participant from the clinical trial is shown in Figure 6.6. The start of each of the three phases and the end of the sit-to-stand transfer are identified using colour coded phase markers.

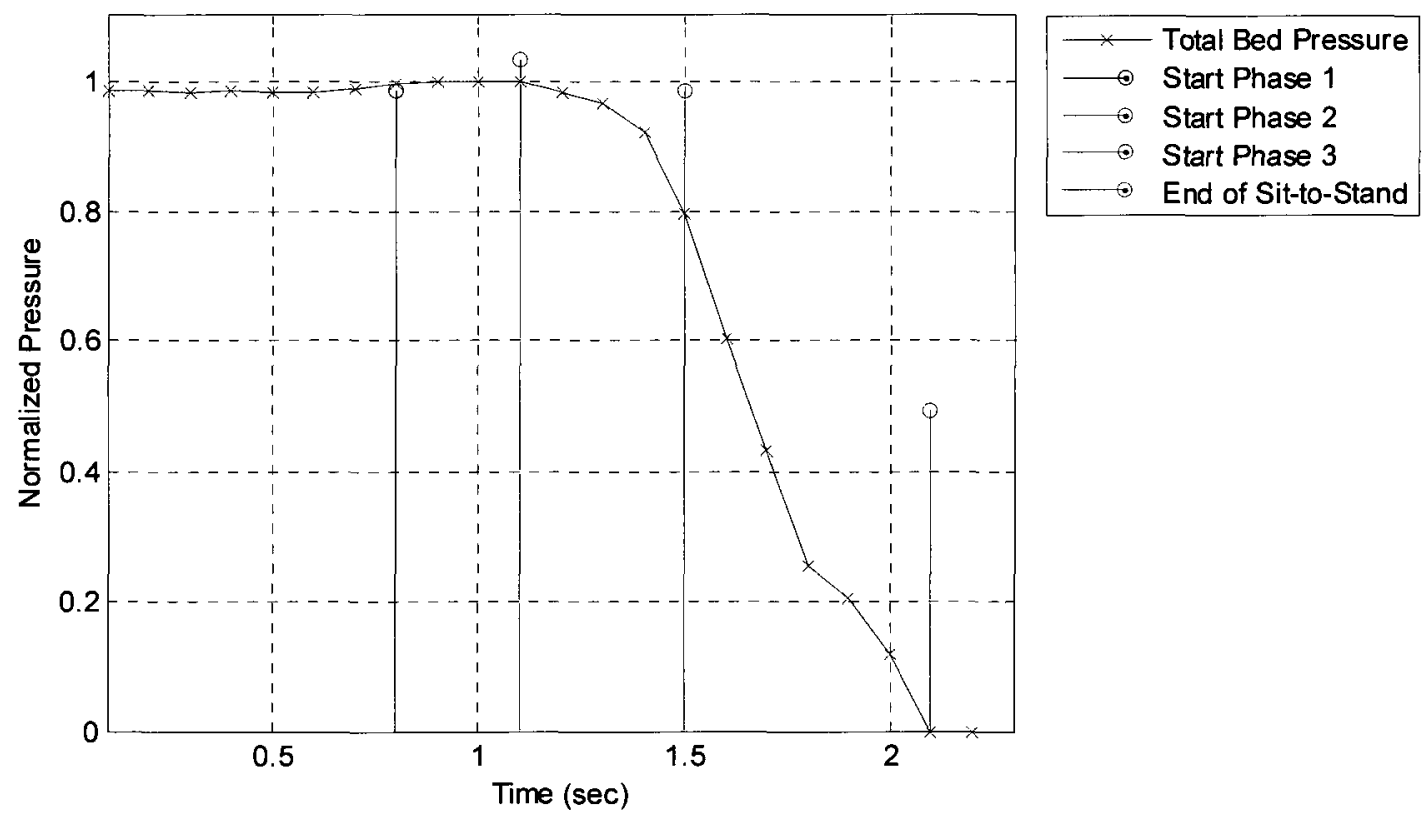

Figure 6.6: Sit-to-stand transfer \#1 for older healthy participant \#3649

The timing information of interest is the length of each phase as well as the total duration of the sit-to-stand cycle. The sit-to-stand phases were extracted using the phase definitions shown in Figure 6.3. From the plot above it can be seen that Phase 1 lasts 0.3 
seconds, phase 2 lasts 0.4 seconds, phase 3 lasts 0.6 seconds and the total sit to stand time is approximately 1.3 seconds. Results showing pressure curves and phase markers for data collected from several other participants during clinical trials will be presented in Section 6.4.

\subsection{Selection of Parameters}

The phase detection algorithm described in Section 6.2.1 depends on three parameters: weight shift, peak and lift which relate to the three phases weight shift, transition and lift described in Section 6.2. Several different values were tested for each in order to select the best parameters to use in the algorithm. The weight shift value was varied from $1 \%$ to $3 \%$, the peak value was varied from 0.75 to 0.95 , and the lift parameter value was varied from $5 \%$ to $10 \%$. All together 30 different parameter sets were tested using the participant data to select the optimal parameters for the algorithm. The parameter values that yielded the best results are: $1 \%$ weight shift, 0.85 peak and $5 \%$ lift. Correct detection of phase start times are extremely important because they directly relate to the timing information extracted for each phase length as well as the total time for the sit-to-stand transfer.

\subsubsection{Weight Shift Parameter Discussion}

In order to optimize the weight shift parameter three different values were tested. The chosen standard was a shift value of $1 \%$. Figure 6.7 shows a sample plot which uses two sets of parameters therefore resulting in the detection of two sets of phase markers. The test parameter set uses an increased weight shift value of $2 \%$ and standard peak and lift 
values. The standard phase markers are displayed using $\square$ 's and the test result phase markers are displayed using *'s.

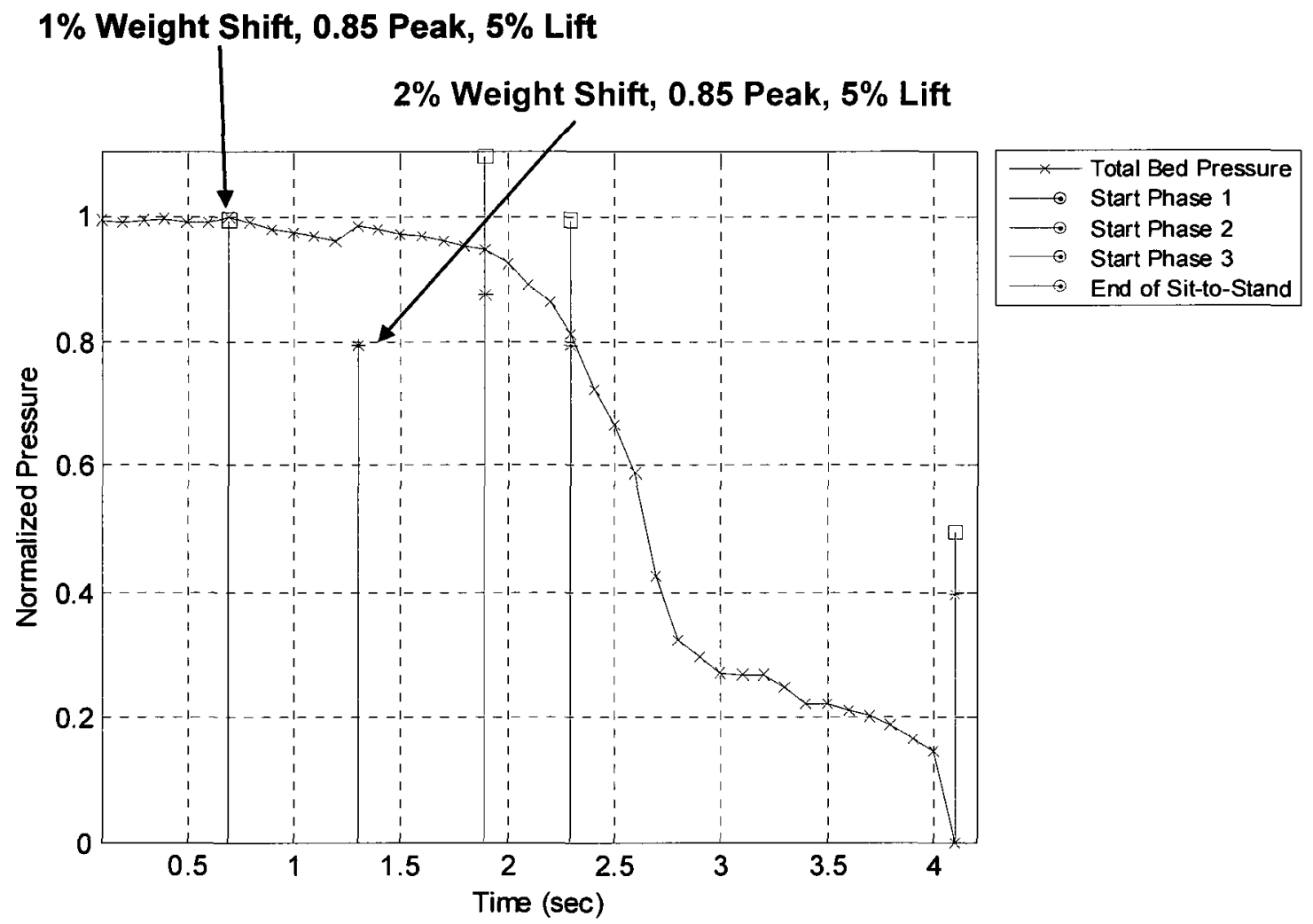

Figure 6.7: Sample plot comparing two different weight shift parameters ( $\square$ 's represent standard results and *'s represent test results)

From the results shown in the figure above it can be seen that due to the increase in the weight shift parameter the phase 1 marker is detected 0.6 seconds later. This affects the phase 1 time as well as the total sit-to-stand time, shortening the length of each by 0.6 seconds.

\subsubsection{Peak Parameter Discussion}

When optimizing the peak parameter used to detect the transition phase five different values, ranging from 0.75 to 0.95 , were tested. The chosen standard was a peak value of 0.85. Figure 6.8 shows a sample plot which compares the test parameter set: $1 \%$ weight 
shift, 0.8 peak and $5 \%$ lift, to the optimal parameter set. The standard phase markers are displayed using $\square$ 's and the test result phase markers are displayed using *'s.

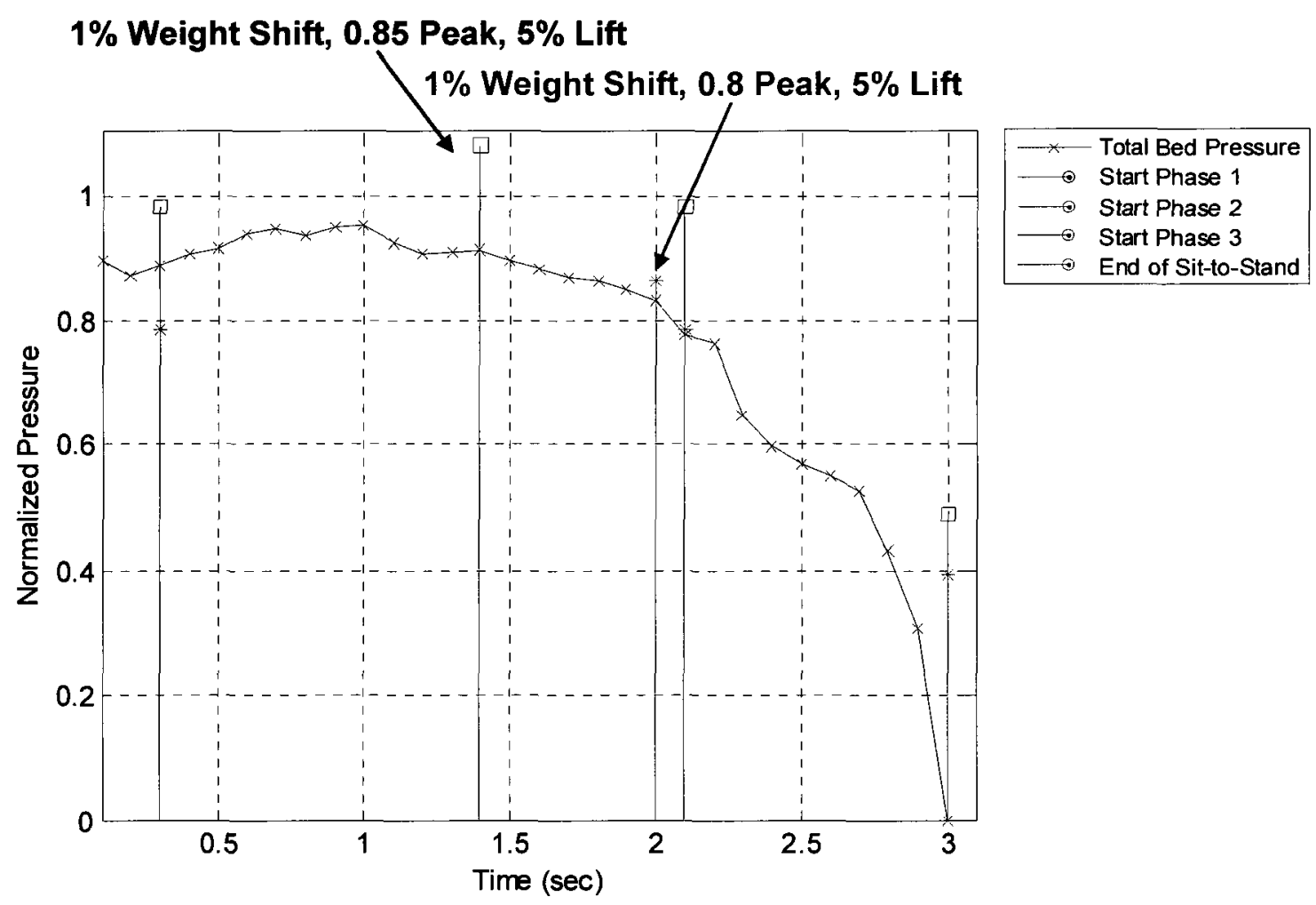

Figure 6.8: Sample plot comparing two different peak parameters

( $\square$ 's represent standard results and *'s represent test results)

From the results shown in the figure above it can be seen that the transition start location has been detected 0.6 seconds later than the standard phase 2 start time. This result affects the phase length time for both the weight shift and transition phases. Phase 1 is increased by 0.6 seconds making it very long and phase 2 is decreased to only 0.1 seconds which is extremely short therefore verifying that the phase 2 test start location is incorrect. 


\subsubsection{Lift Parameter Discussion}

The optimal lift phase parameter was chosen based on results from testing two values, $5 \%$ and $10 \%$. The chosen standard was a lift parameter value of $5 \%$. Figure 6.9 shows a sample plot which compares the test parameter set to the optimal parameter set varying only the lift parameter value. The standard phase markers are displayed using $\square$ 's and the test result phase markers are displayed using *'s.

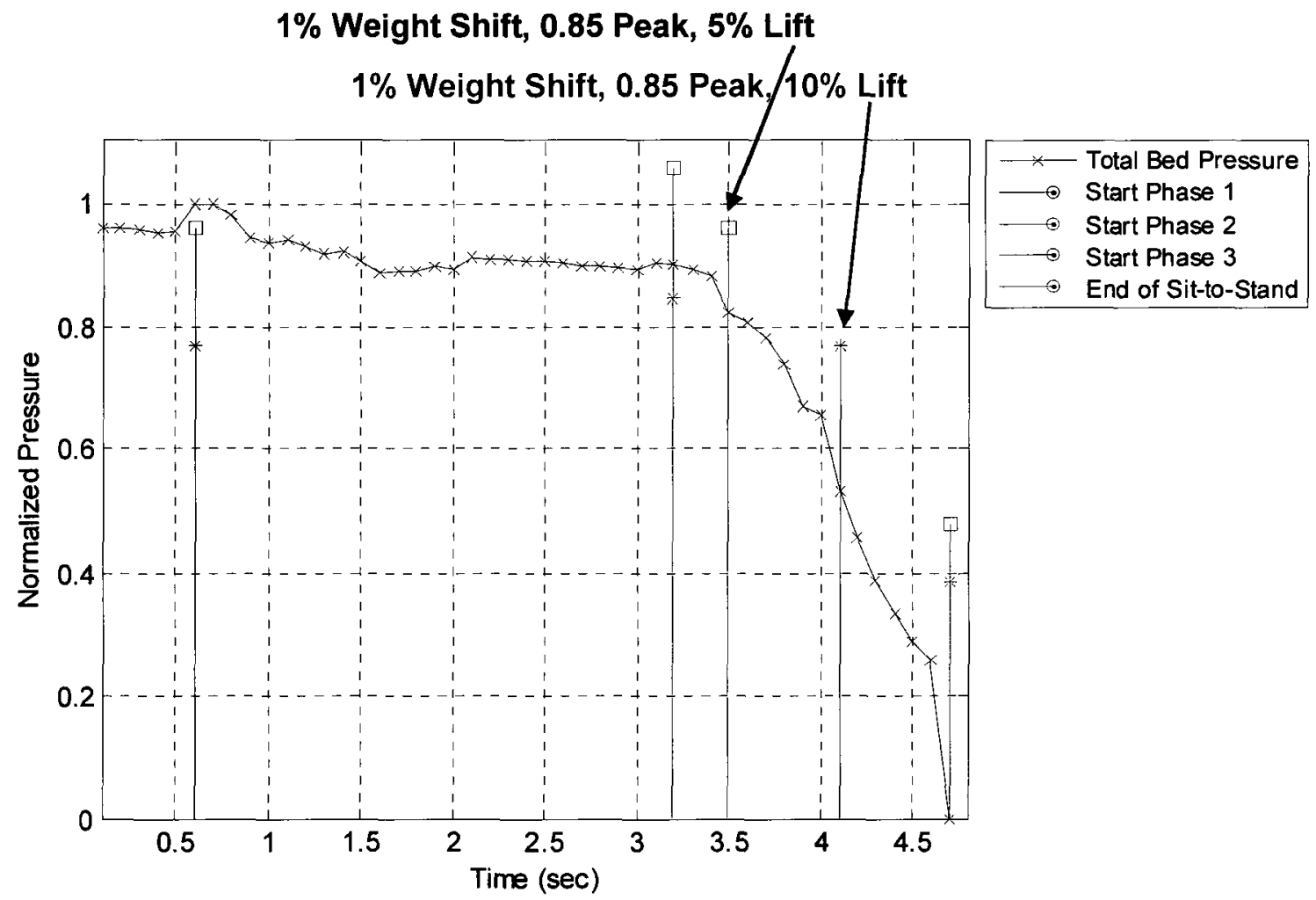

Figure 6.9: Sample plot comparing two different lift parameters

( $\square$ 's represent standard results and *'s represent test results)

From the results shown above it can be seen that the lift phase is detected 0.6 seconds too late. This incorrect phase detection would affect both the time results for the transition and lift phases. The new transition phase time is 0.9 seconds and the new lift phase time is 0.6 seconds compared to a transition phase time of 0.3 seconds and lift phase time of 
1.2 seconds. The test results show inaccuracy because the new transition time is calculated to be longer than the lift time which is incorrect.

Figure 6.10 shows detected phase marker sets resulting from the optimal parameter being compared to a test parameter set where both the weight shift value and peak values are modified.

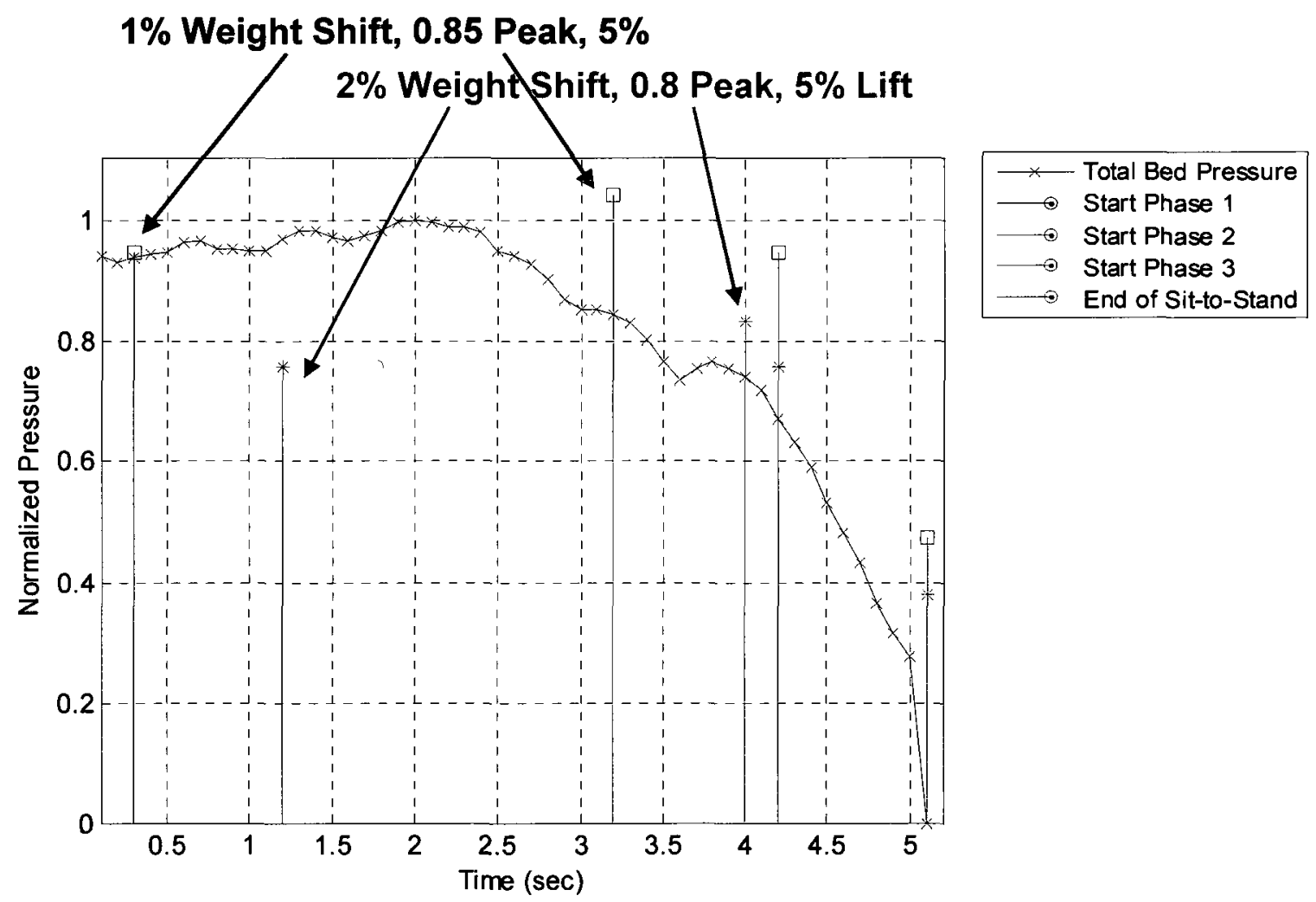

Figure 6.10: Sample plot comparing two different weight shift and peak parameters ( $\square$ 's represent standard results and *'s represent test results)

Varying the weight shift and peak parameters can result in late detection of the weight shift phase 1 and transition phase 2 as shown above.

\subsubsection{Phase 1 Detection Statistics}

The most difficult phase to detect is phase 1 and therefore a good indication of poor results is a low phase 1 detection rate. Sections $6.3 .1-6.3 .3$ individually discussed the 
importance of each parameter value and showed how varying each parameter affected the algorithm results. Table 6.1 below shows the detection rates for all 30 parameter sets when applied to the young healthy participant data. The 30 parameter sets represent each possible three parameter combination that results from varying the weight shift, peak and lift values.

The rows in Table 6.1 correspond to the five different peak values that were tested, the columns correspond to the three weight shift values tested and the sub columns correspond to the two lift values tested. The table is organized into three groups each represented by a different colour. The groups correspond to the weight shift values where $1 \%$ is shown in orange, $2 \%$ is shown in pink and $3 \%$ is shown in blue.

Each entry contains four pieces of information; the largest area calculates the overall detection rate which is displayed as a fraction out of 199; the total 199 represents the number of total young healthy sit-to-stand transfers. The three sub-columns below the overall detection rate indicate the success of each phase marker in terms of correct placement. The phase markers are compared to the standard phase marker results for each participant transfers. The less than column $(<)$ represents phase markers that are detected too early in the sit-to-stand sequence, the greater than $(>)$ column represents phase markers that are detected too late in the sit-to-stand sequence and the equals $(=)$ column represents correctly detected phase markers. 
Table 6.1: Young healthy phase 1 detection success rate

( $>$ represents transfers detected too late, $<$ represents transfers detected too early, and $=$ represents transfers detected correctly)

\begin{tabular}{|c|c|c|c|c|c|c|c|c|c|c|c|c|c|c|c|c|c|c|}
\hline & \multicolumn{6}{|c|}{$\begin{array}{c}\text { Group 1: } \\
\text { Weight Shift: } 1 \% \\
\end{array}$} & \multicolumn{6}{|c|}{$\begin{array}{c}\text { Group 2: } \\
\text { Weight Shift: } 2 \% \\
\end{array}$} & \multicolumn{6}{|c|}{$\begin{array}{c}\text { Group 3: } \\
\text { Weight Shift: } 3 \% \\
\end{array}$} \\
\hline & \multicolumn{3}{|c|}{ Lift: $5 \%$} & \multicolumn{3}{|c|}{ Lift: $10 \%$} & \multicolumn{3}{|c|}{ Lift: $5 \%$} & \multicolumn{3}{|c|}{ Lift: $10 \%$} & \multicolumn{3}{|c|}{ Lift: $5 \%$} & \multicolumn{3}{|c|}{ Lift: $10 \%$} \\
\hline & $>$ & $<$ & $=$ & $>$ & $<$ & $=$ & $>$ & $<$ & $=$ & $>$ & $<$ & $=$ & $>$ & $<$ & $=$ & $>$ & $<$ & $=$ \\
\hline \multirow{2}{*}{$\begin{array}{c}\text { Peak: } \\
0.75\end{array}$} & \multicolumn{3}{|c|}{$196 / 199$} & \multicolumn{3}{|c|}{$196 / 199$} & \multicolumn{3}{|c|}{$181 / 199$} & \multicolumn{3}{|c|}{$181 / 199$} & \multicolumn{3}{|c|}{$152 / 199$} & \multicolumn{3}{|c|}{$152 / 199$} \\
\hline & 0 & 0 & 196 & 0 & 0 & 196 & 36 & 68 & 77 & 36 & 86 & 77 & 26 & 74 & 52 & 26 & 74 & 52 \\
\hline \multirow{2}{*}{$\begin{array}{c}\text { Peak: } \\
0.80\end{array}$} & \multicolumn{3}{|c|}{$196 / 199$} & \multicolumn{3}{|c|}{$196 / 199$} & \multicolumn{3}{|c|}{$181 / 199$} & \multicolumn{3}{|c|}{$181 / 199$} & \multicolumn{3}{|c|}{$152 / 199$} & \multicolumn{3}{|c|}{$152 / 199$} \\
\hline & 0 & 0 & 196 & 0 & 0 & 196 & 36 & 68 & 77 & 36 & 86 & 77 & 26 & 74 & 52 & 26 & 74 & 52 \\
\hline \multirow{2}{*}{$\begin{array}{c}\text { Peak: } \\
0.85\end{array}$} & \multicolumn{3}{|c|}{$\begin{array}{c}\text { Original: } \\
196 / 199 \\
\end{array}$} & \multicolumn{3}{|c|}{$196 / 199$} & \multicolumn{3}{|c|}{$181 / 199$} & \multicolumn{3}{|c|}{$181 / 199$} & \multicolumn{3}{|c|}{$152 / 199$} & \multicolumn{3}{|c|}{$152 / 199$} \\
\hline & $\mathbf{0}$ & $\mathbf{0}$ & 196 & 0 & 0 & 196 & 36 & 68 & 77 & 36 & 86 & 77 & 26 & 74 & 52 & 26 & 74 & 52 \\
\hline \multirow{2}{*}{$\begin{array}{c}\text { Peak: } \\
0.90\end{array}$} & \multicolumn{3}{|c|}{$195 / 199$} & \multicolumn{3}{|c|}{$195 / 199$} & & $31 / 1$ & & & $1 / 1$ & & & $52 / 1$ & & & $2 / 1$ & \\
\hline & 0 & 1 & 194 & 0 & 1 & 194 & 36 & 69 & 76 & 36 & 87 & 76 & 26 & 75 & 51 & 26 & 75 & 51 \\
\hline & & $95 /$ & & & 195 & & & $31 / 1$ & & & $1 / 1$ & & & $52 / 1$ & & & $2 / 1$ & \\
\hline & 0 & I & 194 & 0 & 1 & 194 & 36 & 69 & 76 & 36 & 87 & 76 & 26 & 75 & 51 & 26 & 75 & 51 \\
\hline
\end{tabular}

From the table above it can be seen that as the Weight Shift parameter is increased from $1 \%$ to $3 \%$ the phase 1 detection rate decreases from $98 \%$ to $91 \%$ to a $76 \%$ detection rate. However at the same time the correct placement and therefore length of phase 1 , shown in the '=' column in bold, decrease from $98 \%$ to $39 \%$ to a $26 \%$ success rate. Examining the three correct placement columns for the various weight shift values it shows that as the correct placement rate decreases the late phase 1 detection rate significantly increases. 
Therefore the incorrect placement of the phase 1 marker will most often result in a lowered sit-to-stand transfer time as shown in Table 6.2 below.

Table 6.2: Young healthy participant average sit-to-stand time using test parameters

\begin{tabular}{|l|c|c|c|c|c|c|}
\hline & \multicolumn{2}{|c|}{$\begin{array}{c}\text { Group 1: } \\
\text { Weight Shift: 1\% }\end{array}$} & \multicolumn{2}{c|}{$\begin{array}{c}\text { Group 2: } \\
\text { Weight Shift: 2\% }\end{array}$} & \multicolumn{2}{c|}{$\begin{array}{c}\text { Group 3: } \\
\text { Weight Shift: 3\% }\end{array}$} \\
\cline { 2 - 7 } & Lift: 5\% & Lift: 10\% & Lift: 5\% & Lift: 10\% & Lift: 5\% & Lift: 10\% \\
\hline Peak: 0.75 & $0.92 \mathrm{sec}$ & $0.92 \mathrm{sec}$ & $0.83 \mathrm{sec}$ & $0.83 \mathrm{sec}$ & $0.75 \mathrm{sec}$ & $0.75 \mathrm{sec}$ \\
\hline Peak: 0.80 & $0.92 \mathrm{sec}$ & $0.92 \mathrm{sec}$ & $0.83 \mathrm{sec}$ & $0.83 \mathrm{sec}$ & $0.75 \mathrm{sec}$ & $0.75 \mathrm{sec}$ \\
\hline Peak: 0.85 & $\begin{array}{c}\text { Original: } \\
\mathbf{0 . 9 2} \mathrm{sec}\end{array}$ & $0.92 \mathrm{sec}$ & $0.83 \mathrm{sec}$ & $0.83 \mathrm{sec}$ & $0.75 \mathrm{sec}$ & $0.75 \mathrm{sec}$ \\
\hline Peak: 0.90 & $0.92 \mathrm{sec}$ & $0.92 \mathrm{sec}$ & $0.83 \mathrm{sec}$ & $0.83 \mathrm{sec}$ & $0.75 \mathrm{sec}$ & $0.75 \mathrm{sec}$ \\
\hline Peak: 0.95 & $0.92 \mathrm{sec}$ & $0.92 \mathrm{sec}$ & $0.83 \mathrm{sec}$ & $0.83 \mathrm{sec}$ & $0.75 \mathrm{sec}$ & $0.75 \mathrm{sec}$ \\
\hline
\end{tabular}

\subsection{Clinical Results}

During the clinical trial conducted in the summer of 2008 at the Élisabeth Bruyère facility data was collected from 25 recruited participants. Within this participant group there were ten healthy young subjects, five healthy older subjects, five post-stroke subjects and five post-hip fracture subjects. The healthy participants performed up to twenty transfers each and the functionally impaired participants performed up to ten transfers each. Figure 6.11 - Figure 6.14 show sample plots from each of the four participant groups. Each plot shows the normalized pressure signal representing the pressure applied to the 
bed during the sit-to-stand transfer sequence, start phase markers for phases $1,2,3$ and an end of sit-to-stand transfer marker.

\subsubsection{Sample Young Healthy Results}

The sit-to-stand data collected from the group of 10 young healthy participants all showed very similar results. Figure 6.11 shows one sit-so-stand transfer performed by a young healthy participant.

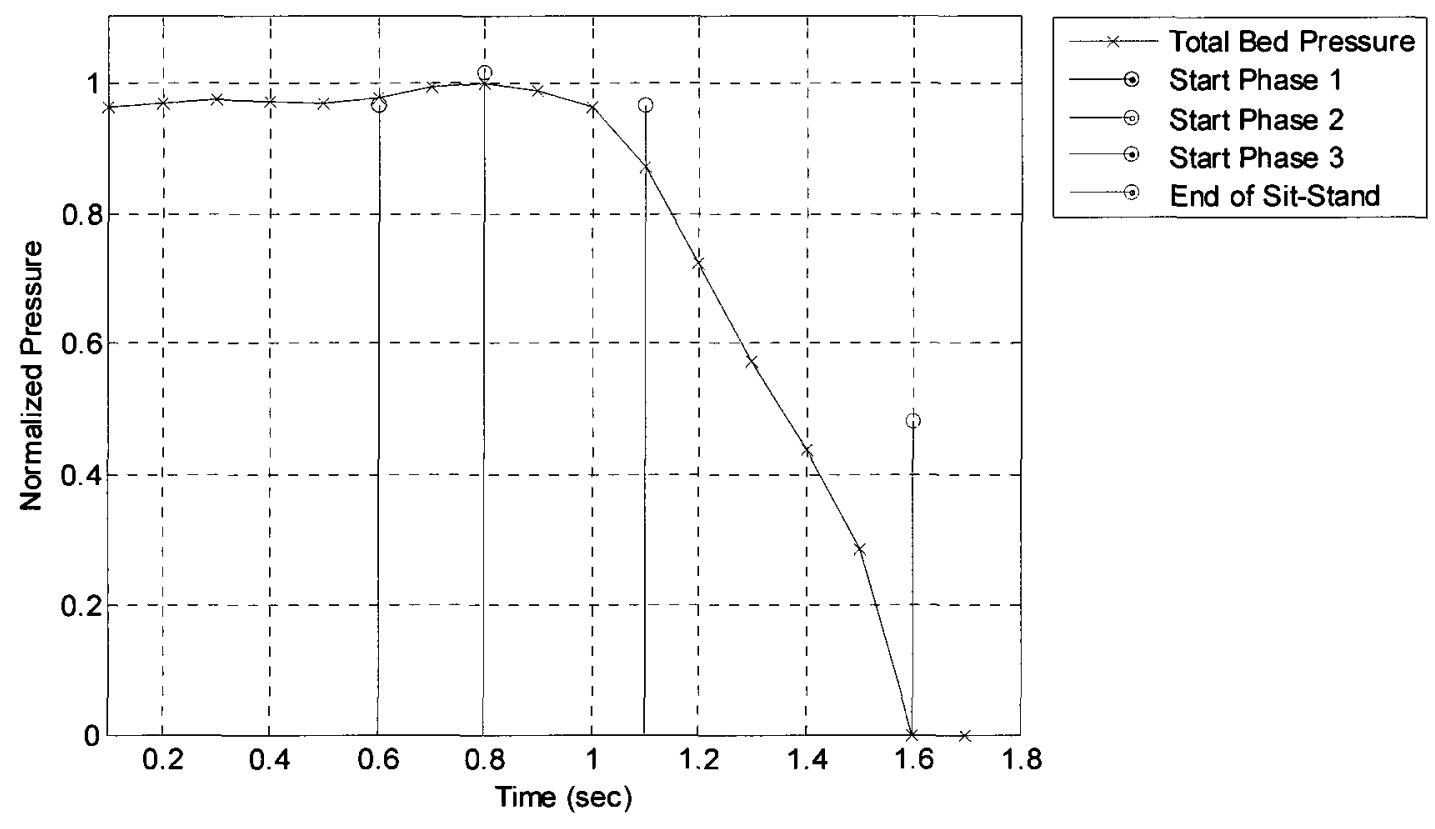

Figure 6.11: Sit-to-stand transfer \#12 for young healthy participant \#2164

The young healthy participant result shown above is a very good example of an ideal sit-to-stand transfer. The pressure curve and phase markers compare extremely well with the simulated pressure curve shown in Figure 6.4. From the figure above it can be seen that the entire sit-to-stand time is approximately 1.0 seconds which is extremely fast. Breaking down this sit-to-stand sequence into phases, as defined in Figure 6.3, results in a weight shift (phase 1) time of 0.2 seconds, transition (phase 2) time of 0.3 seconds, and lift (phase 3) time of 0.5 seconds. 


\subsubsection{Sample Older Healthy Results}

The older healthy participant group was made up of 5 subjects all of which were over the age of 65 . The older healthy sit-to-stand transfers showed slightly more variety than the young healthy but the majority of sequences performed were similar to the sample transfer shown in Figure 6.12.

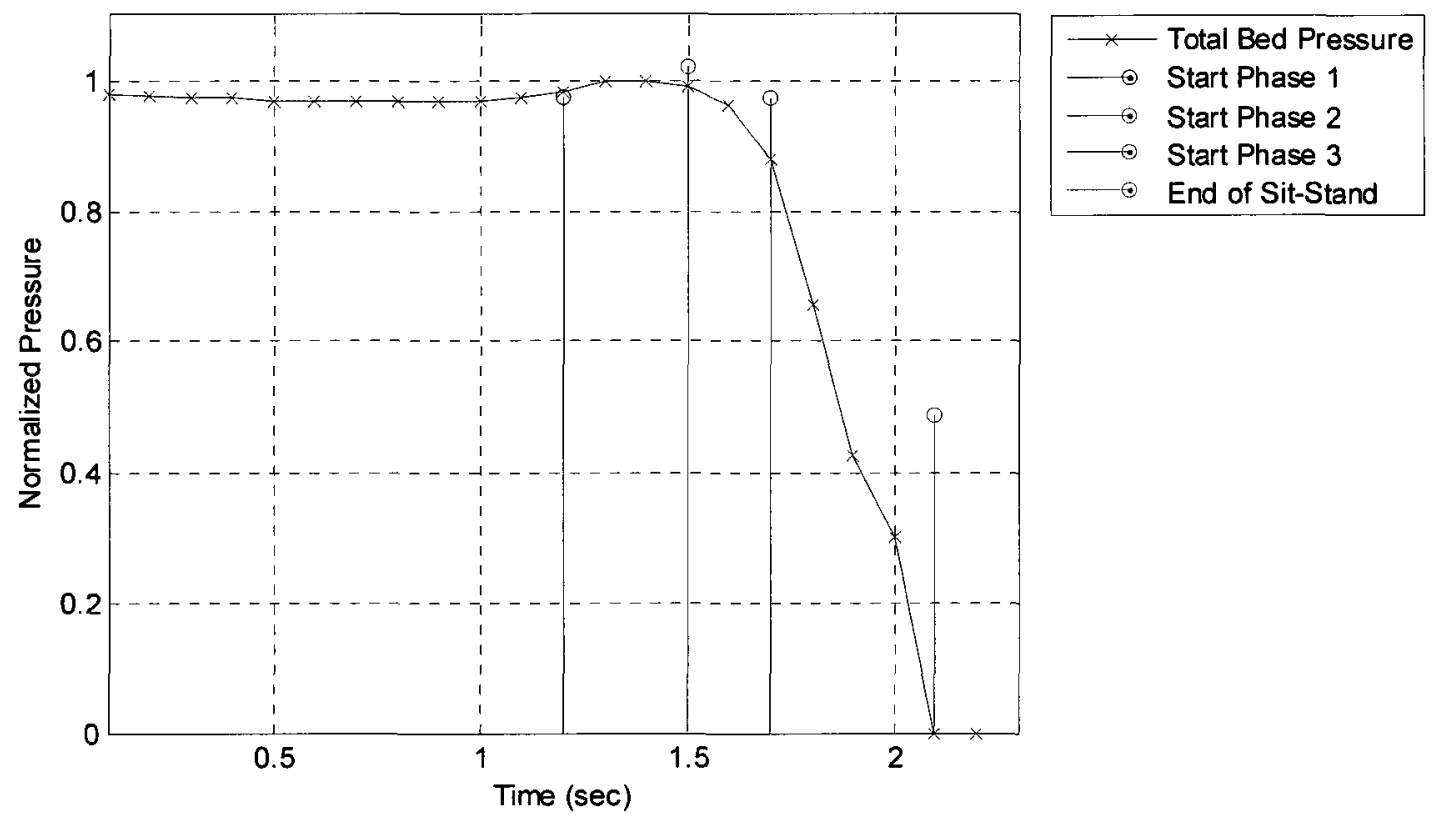

Figure 6.12: Sit-to-stand transfer \#23 for older healthy participant \#5265

The pressure curve and phase markers shown in Figure 6.12 are also a very good example of an ideal sit-to-stand transfer. The pressure reflects the weight shift, transition and lift movements described in detail in Figure 6.3. The sit-to-stand sequence is completed in approximately 0.9 seconds with the weight shift (phase 1) lasting 0.3 seconds, the transition (phase 2) lasting 0.2 seconds and the lift (phase 3) lasting 0.4 seconds. 


\subsubsection{Sample Older Post-Stroke Results}

The post-stroke participant group was made up of 5 subjects all of which were at least 65 years of age and although had experienced a stroke in the past were currently medically stable. Each participant was able to perform the sit-to-stand transfer independently. The pressure signals representing the sit-to-stand transfers varied greatly from transfer to transfer within one subject as well as from subject to subject; a sample result is shown in Figure 6.13.

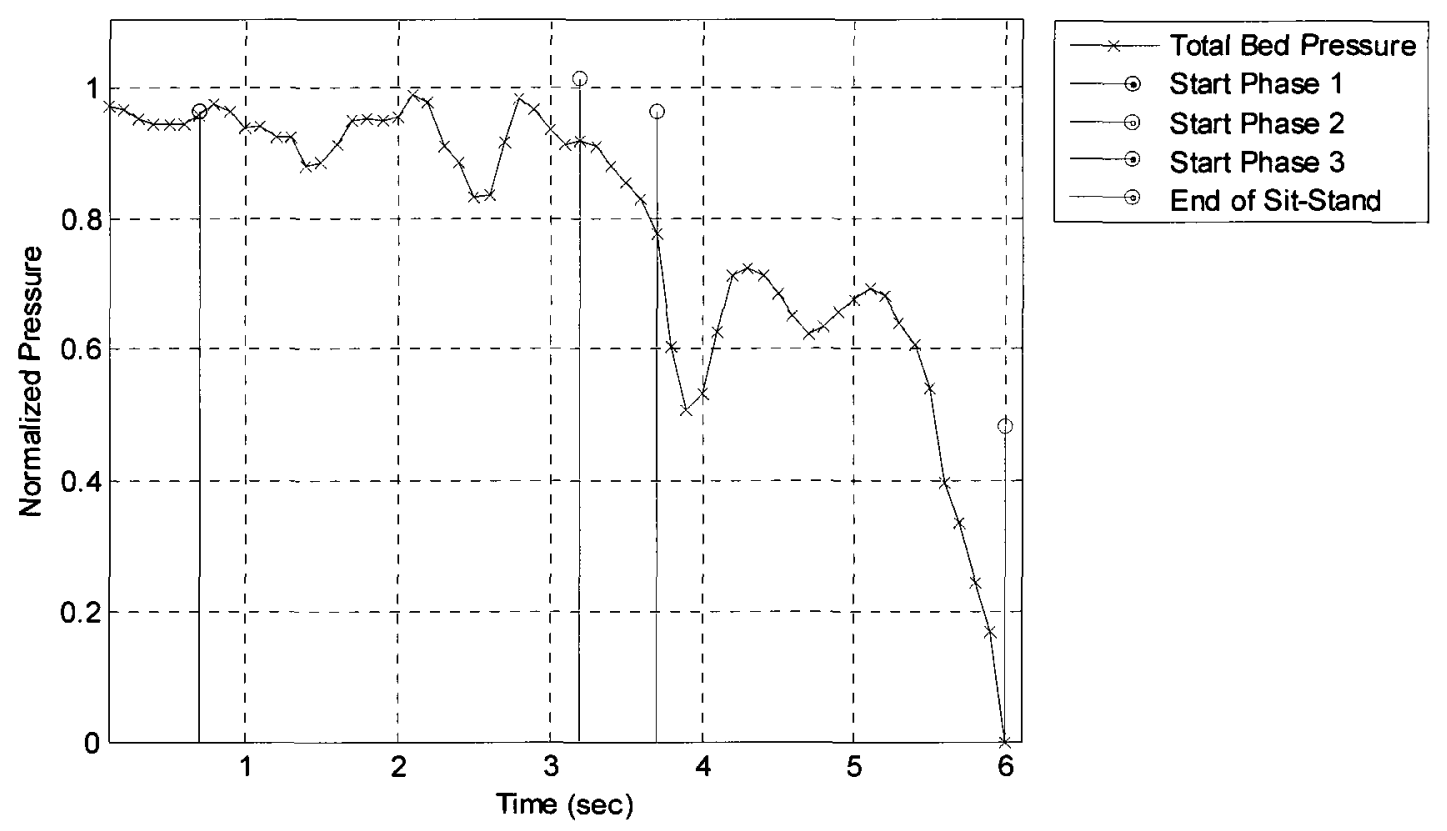

Figure 6.13: Sit-to-stand transfer \#2 for post-stroke participant \#6996

Comparing the above sit-to-stand sequence to the ideal sit-to-stand sequence shows many differences. The pressure signal is rapidly increasing and decreasing and the sit-tostand transfer lasts approximately 5.3 seconds which is much longer than the healthy participant results shown in Sections 6.4.1 and 6.4.2. The weight shift (phase 1) time is 2.5 seconds, the transition (phase 2) time is 0.5 seconds and the lift (phase 3 ) time is 2.3 seconds. 


\subsubsection{Sample Older Post-Hip Results}

The post-hip participant group was made up of 5 hip fracture subjects who had recently had surgery to repair a fractured hip and were over the age of 65 . Each participant was medically stable and was able to perform the sit-to-stand transfer independently. The sitto-stand transfers varied greatly between subjects but were found to have more similarities within each participant when compared to the post-stroke participants. A sample post-hip sit-to-stand transfer is shown in Figure 6.14 .

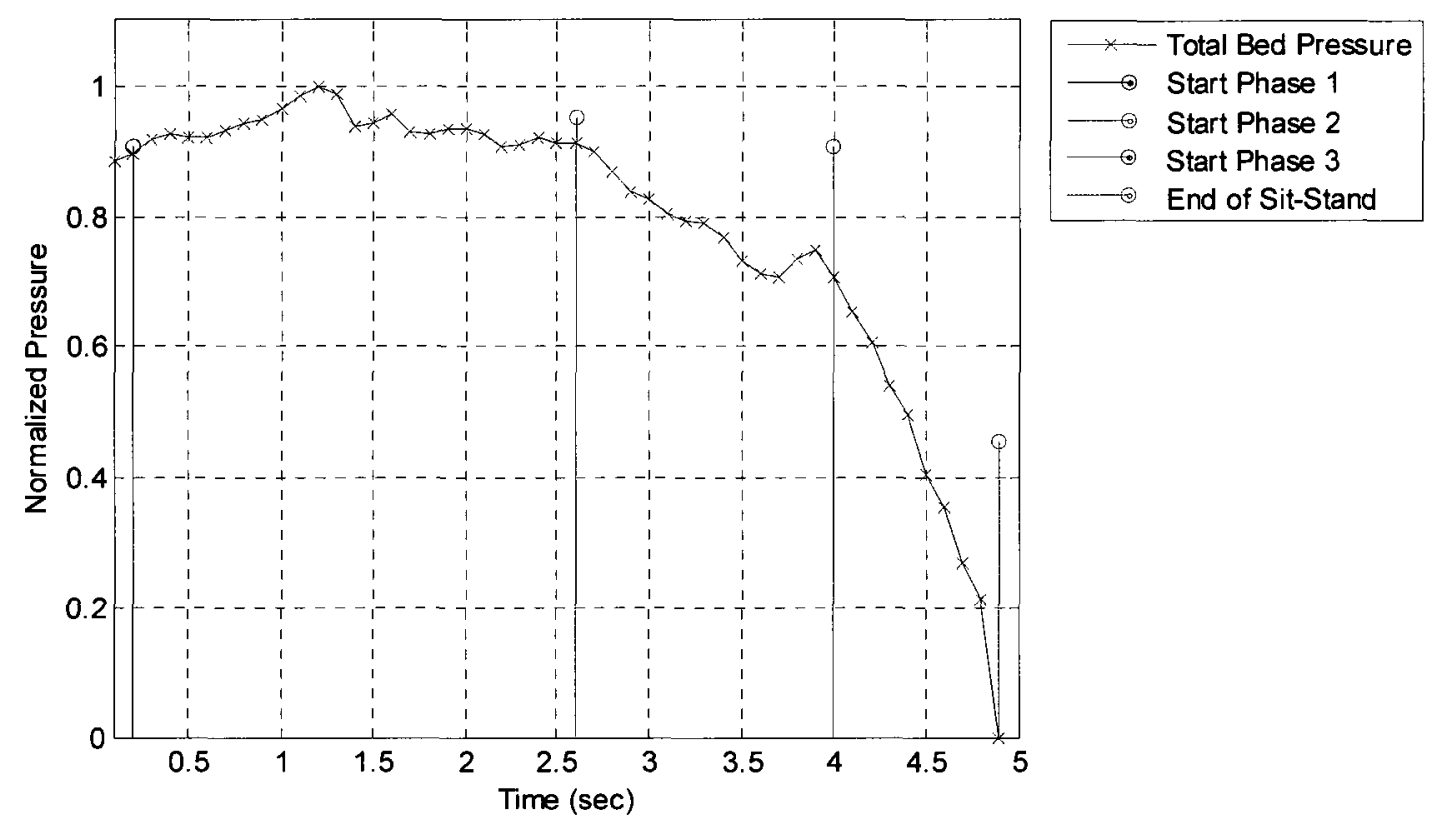

Figure 6.14: Sit-to-stand transfer $\# 8$ for post-hip participant \#8144

The pressure signal above differs greatly from the ideal sit-to-stand pressure signal but is much smoother than the post-stroke pressure signal shown in Figure 6.13. The sitto-stand transfer is approximately 4.7 seconds long and is made up of weight shift (phase 1) time of 2.4 seconds, transition (phase 2) time of 1.4 seconds and lift (phase 3 ) time of 0.9 seconds. 


\subsection{Validating Results}

The algorithm results presented in Section 6.4 were compared to results published by Arcelus et al. [72] in order to validate their accuracy. Total sit-to-stand times were compared using data from all ten young healthy participants. The main difference between the method presented in this chapter and the published journal paper is the use of a high resolution pressure sensitive floor plate. The sit-to-stand timing results published by Arcelus et al. were produced using a greater amount of processing in order to examine both the bed pressure data and floor pressure data and overcome the problem of synchronizing the data from both. The algorithm presented in this chapter is processed much faster because it extracts the sit-to-stand transfer timing information using only the bed pressure mat technology. Using only the bed mats is a more feasible solution when considering having to install this type of equipment into people's homes. The floor mat is a much more expensive technology and elderly people could trip on it therefore causing it to be a potential safety hazard.

Figure 6.15 shows the individual transfer times for each participant represented by *'s as well as the average sit-to-stand time for each participant represented by o's. 


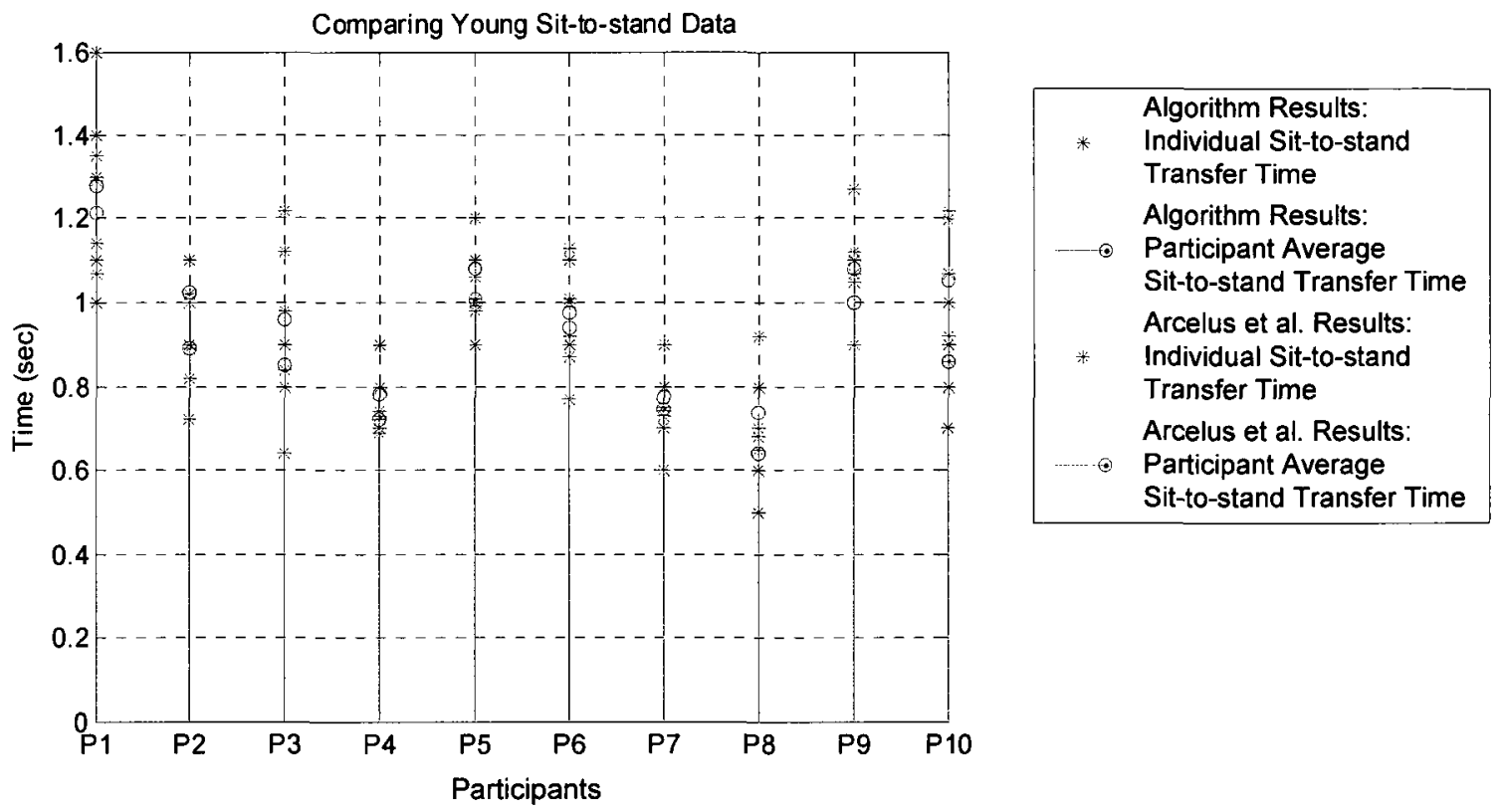

Figure 6.15: Comparing participant results to validate algorithm

The results compare well and a less crowded comparison is shown in Figure 6.16 where only the average times for each participant are displayed.

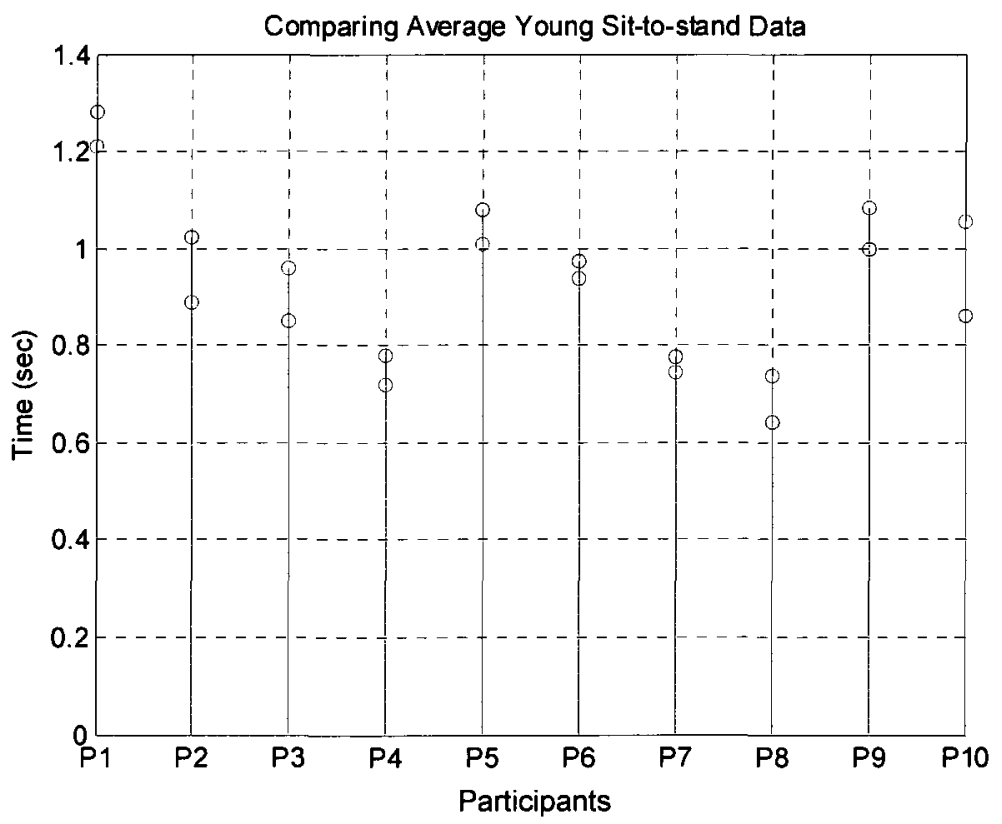

Algorithm Results:

Participant Average Sit-to-stand Transfer Time Arcelus et al. Results:

Participant Average Sit-to-stand Transfer Time

Figure 6.16: Comparing average sit-to-stand times for each participant 
The overall young healthy sit-to-stand average time calculated using the algorithm presented is 0.92 seconds and the average time published by Arcelus et al. [72] is 0.94 seconds. Therefore the average sit-to-stand time result for the young healthy participant group compares very well. The error between the individual participant average sit-tostand times varies from 0.04 seconds to 0.19 seconds with a standard deviation of 0.046 .

As a final comparison the first five sit-to-stand transfer times performed by one young healthy participant were examined. The results comparing individual transfers for one young participant are shown in Figure 6.17 below.

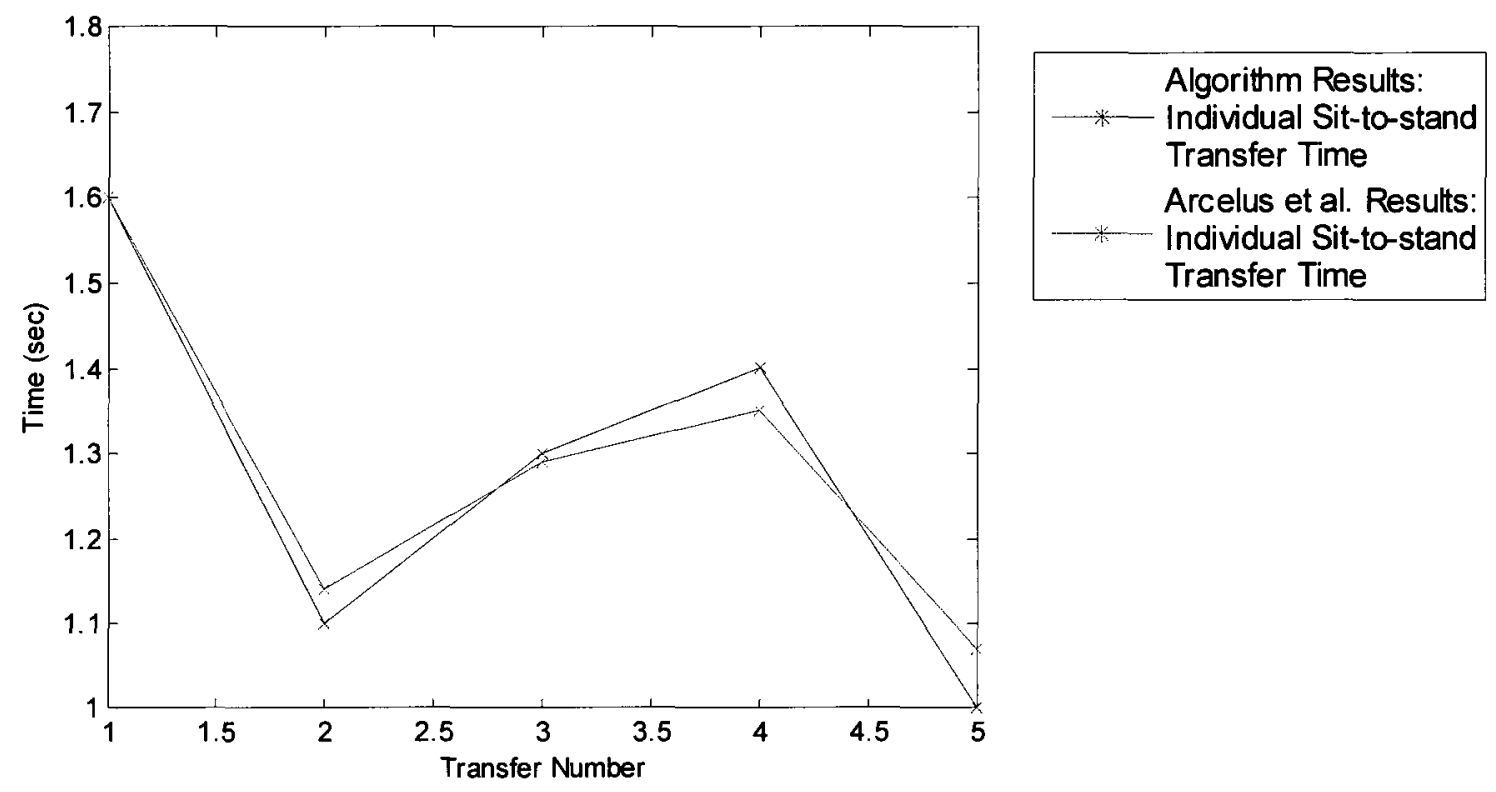

Figure 6.17: Comparing sit-to-stand times for individual transfers

The results above show a good comparison between the algorithm results presented in Section 6.4 and the Arcelus et al. [72] published results. The similar trends in both individual sit-to-stand transfer times and average participant transfer times validate the results produced by the presented phase detection algorithm. 


\subsection{Bounce Detection for Functionally Impaired Participants}

From the older post-stroke and older post-hip samples shown earlier in Figure 6.13 and

Figure 6.14 it can be seen that the phase 1 length is much longer when compared to the young and older healthy subjects. An older stroke sit-to-stand example transfer is shown in Figure 6.18 below.

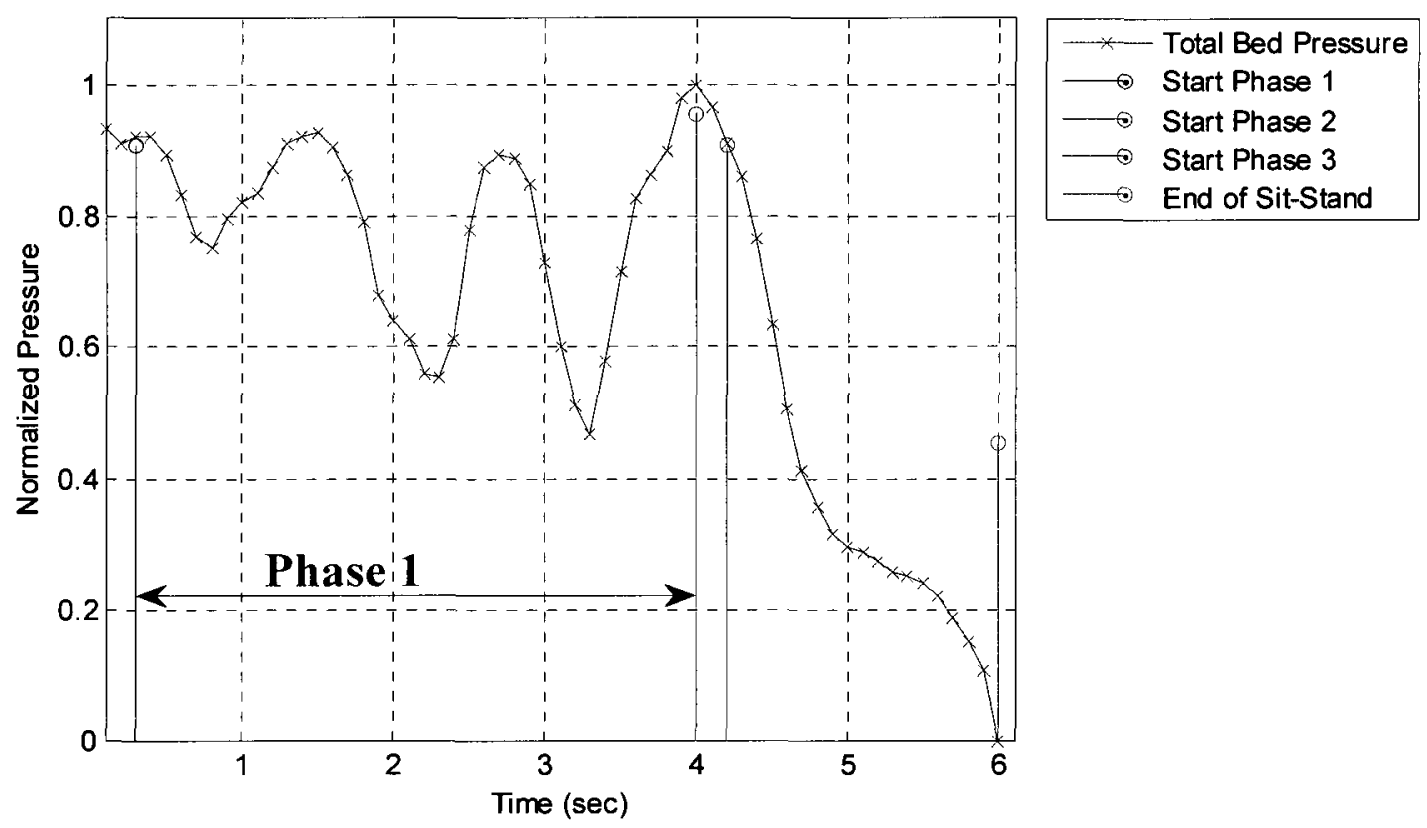

Figure 6.18: Sit-to-stand transfer \#1 for post-stroke participant \#1269

In the older post-stroke sample shown above the phase 1 length is very long, making up the bulk of the sit-to-stand transfer time, during this phase the normalized pressure is continuously changing. This type of movement is seen only in some of the functionally impaired participants because the task of performing the sit-to-stand sequence is much more difficult for them when compared to the healthy participants. From looking at the videos it is seen that these participants often shuffle or bounce while they are trying to perform the sit-to-stand sequence and the next chapter section will look at an algorithm which helps to identify these types of movements. 


\subsubsection{Algorithm Description}

The three types of movements that the algorithm is looking to detect are shuffling, bouncing and unsuccessful transfer. The algorithm analyzes the normalized pressure signal searching for a valley situated between two peaks. The three different types of movements have been assigned corresponding valley depths. Shuffling is detected if there is a $10-19 \%$ dip in the pressure signal, bouncing is detected if there is a $20-49 \%$ dip in the signal and an unsuccessful transfer is detected if there is a greater than $50 \%$ dip in the signal. Figure 6.19 shows a sample transfer for each one of the three types of movements.

\section{Shuffling}

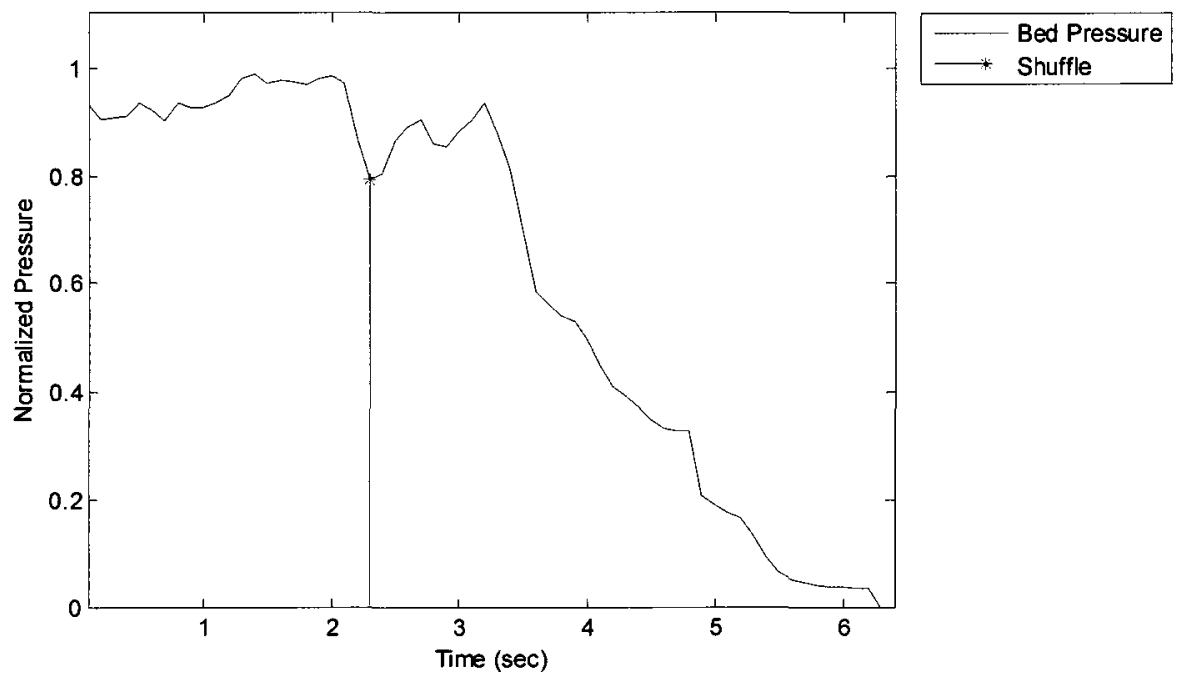

(a) - Participant transfer with a shuffle detected 


\section{Bouncing}

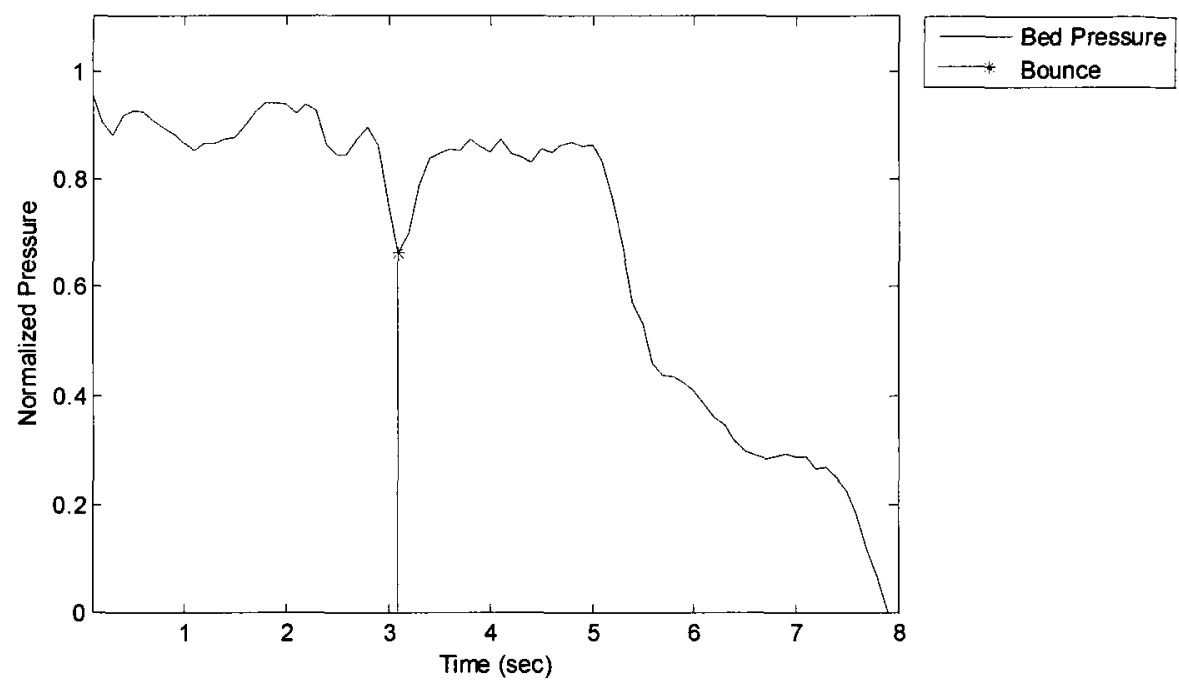

(b) - Participant transfer with a bounce detected

3. Unsuccessful

Transfer

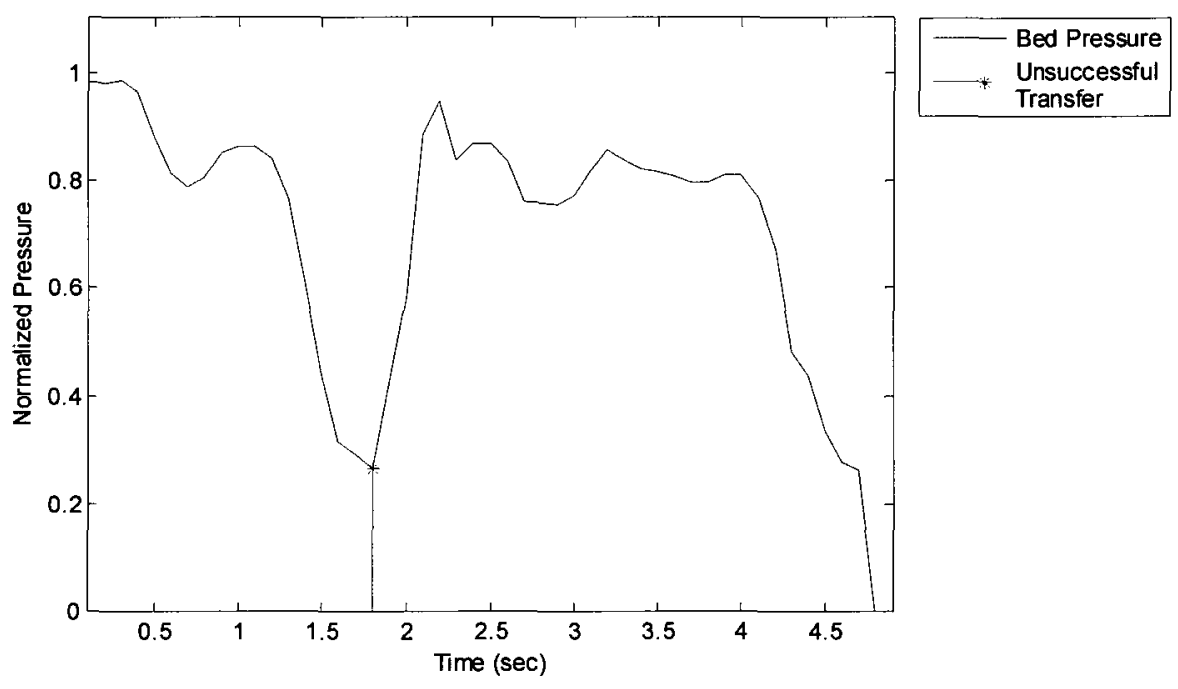

(c) - Participant transfer with a unsuccessful transfer detected

Figure 6.19: Sample plots for functionally impaired participant movements

The samples shown above are sit-to-stand transfers performed by post-stroke and post-hip participants. 


\subsubsection{Functionally Impaired Participant Results}

All of the sit-to-stand transfers performed by functionally impaired participants were processed using the bounce detection algorithm and results for two transfers performed by the same participant are shown in Figure 6.20 and Figure 6.21 .

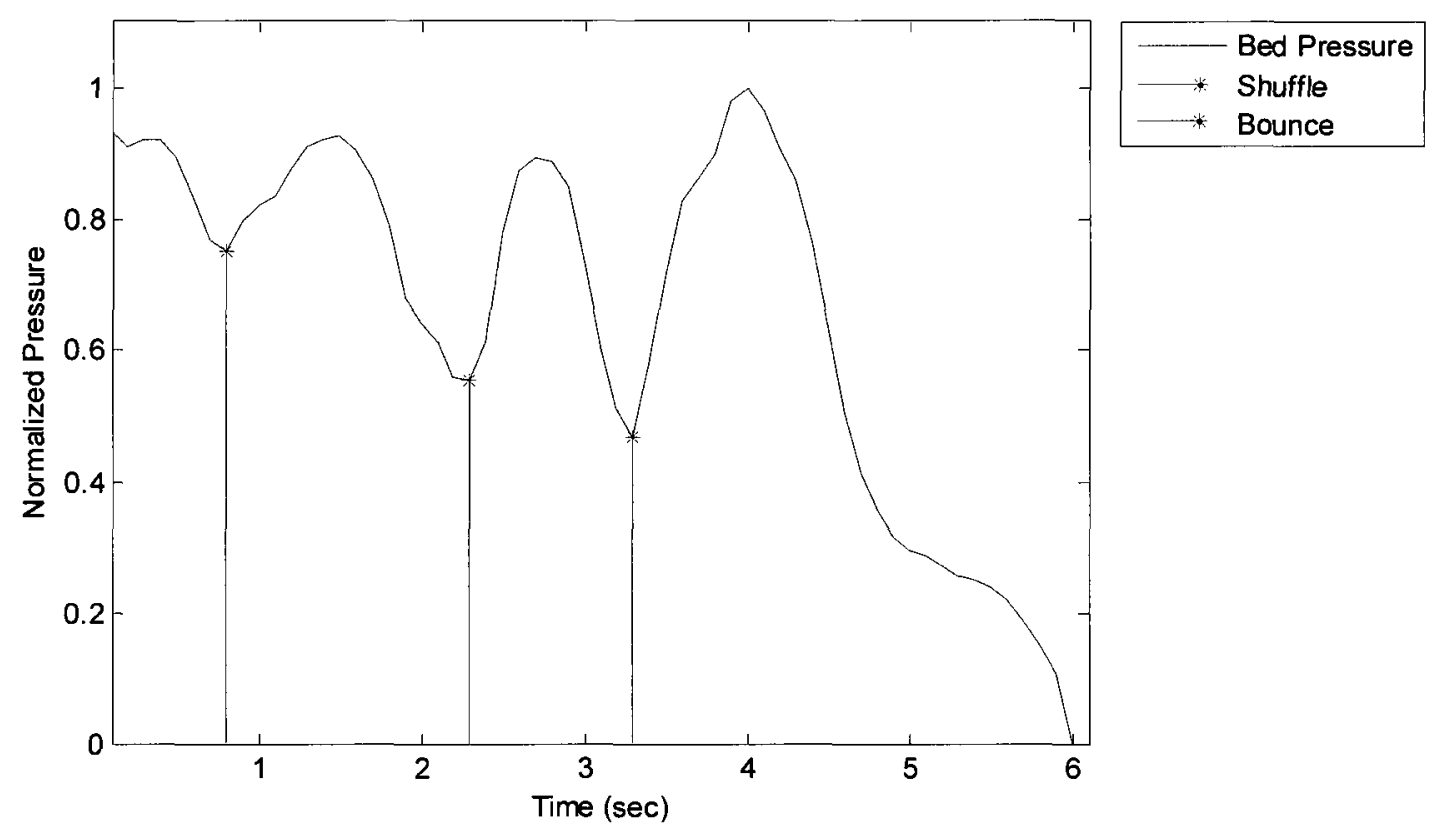

Figure 6.20: Sit-to-stand transfer \#1 for post-stroke participant \#1269

During the sit-to-stand transfer shown above two cases of bouncing and one shuffle are detected. This subject is unable to transition from sitting quietly to lifting off of the bed smoothly. Instead the subject generates momentum by shuffling their hips forwards and by transferring their weight back and forth from the feet to the hips while rock their trunk forwards and backwards. Once the subject has generated enough momentum the lift portion of the sit-to-stand transfer is completely very quickly.

Another sit-to-stand transfer performed by the same subject is shown in Figure 6.21 below. 


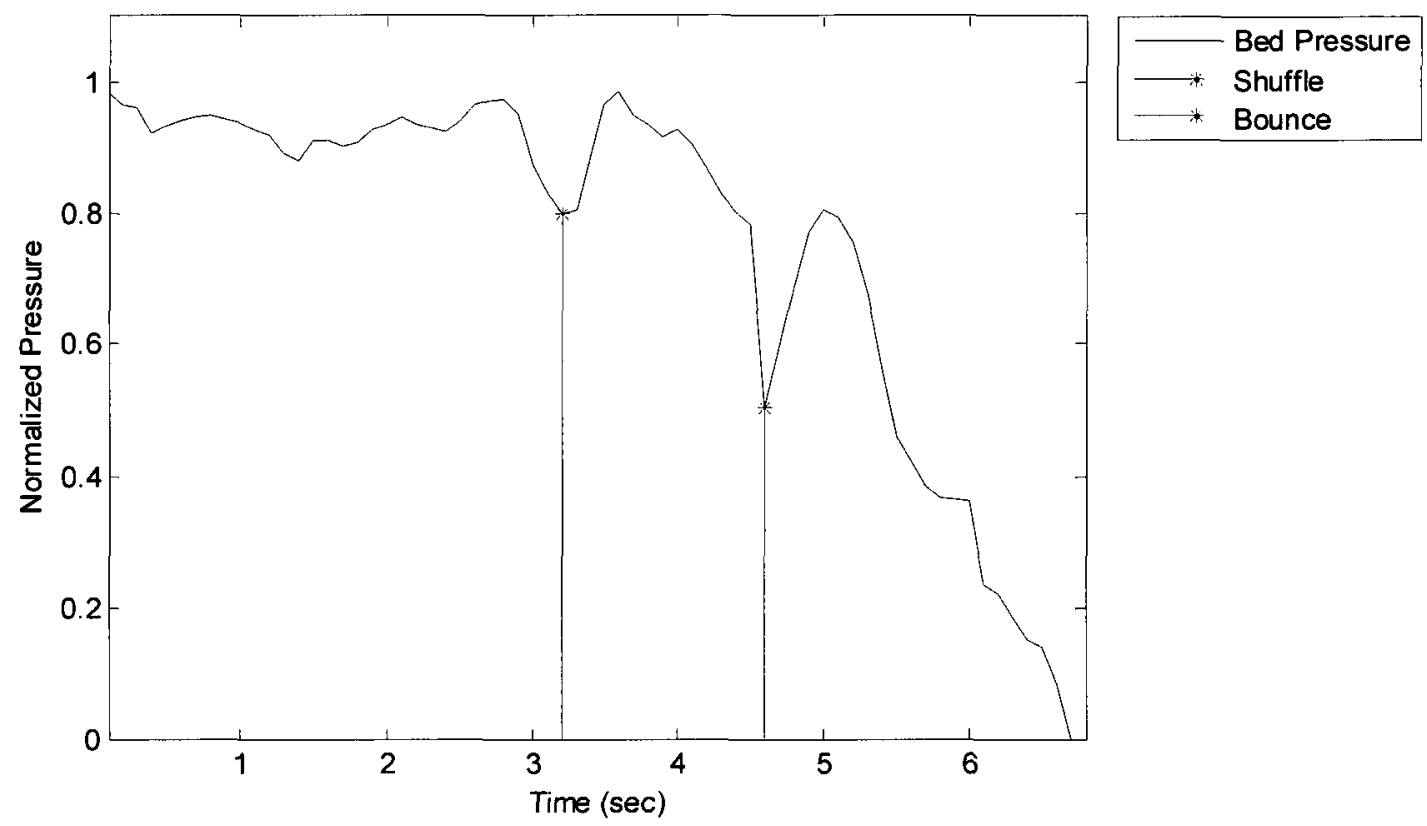

Figure 6.21: Sit-to-stand transfer \#8 for post-stroke participant \#1269

The bounce detection algorithm detected a shuffle followed by a bounce when analyzing the sit-to-stand transfer shown above. In this case the shuffle is used to generate momentum and the bounce occurs during the end portion of the sit-to-stand sequence. Both Figure 6.20 and Figure 6.21 are examples of unstable sit-to-stand sequences because they differ so greatly from the ideal sequence presented in Figure 6.4.

Analyzing all sit-to-stand transfers performed by one participant using the bounce detection algorithm can be used to identify whether a participant follows a certain trend when performing a number of consecutive sit-to-stand transfers. A summary plot was generated for each participant showing the number of movements and types of movements detected during each transfer. A sample summary plot, generated from the participant whose two individual transfers were presented in Figure 6.20 and Figure 6.21, is shown in Figure 6.22. 


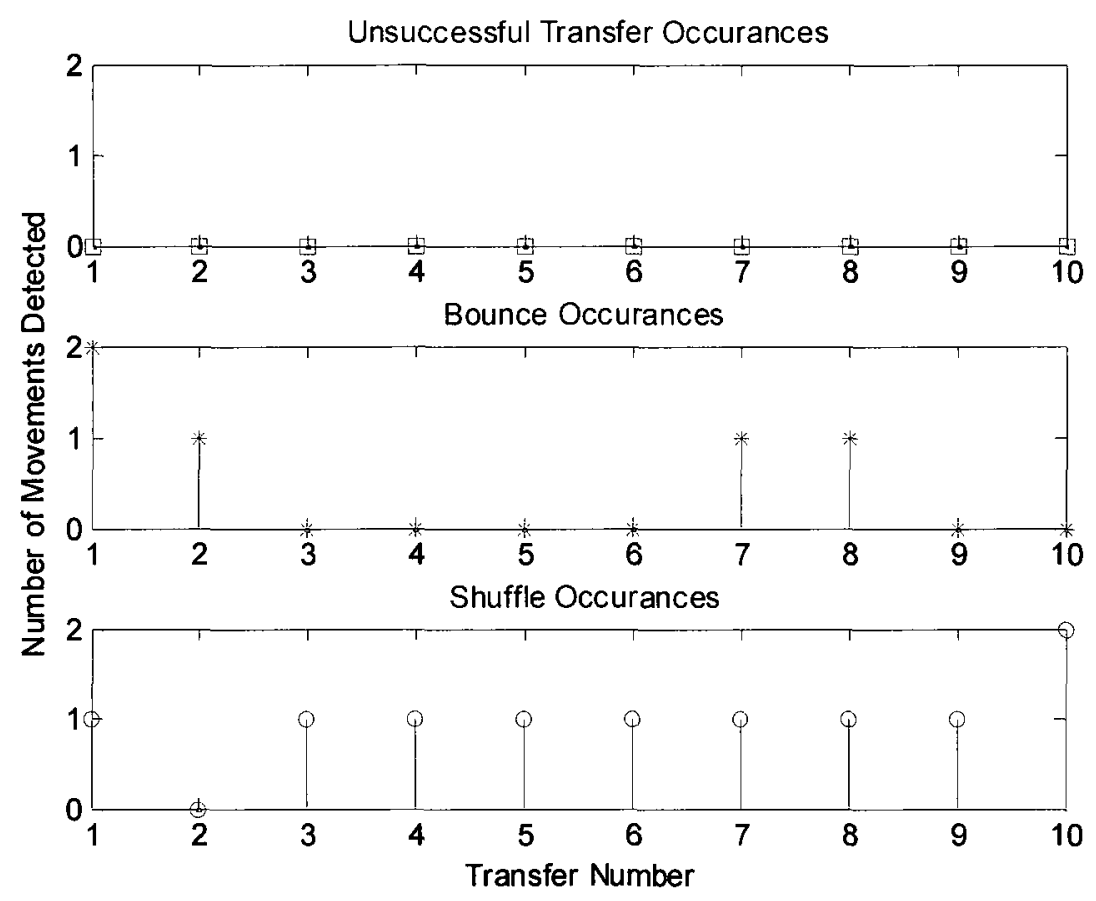

Figure 6.22: Post-stroke participant \# 1269 movement summary

The summary plot shows the 10 sit-to-stand transfers performed by the post-stroke participant in order. The detected movements shown in Figure 6.20 correspond to transfer number one in the above plot and the detected movements shown in Figure 6.21 correspond to transfer number eight in the above plot. The summary plot for this participant shows that shuffle movements are detected throughout $90 \%$ of the subjects' transfers and bouncing is detected in $40 \%$ of the transfers. Clinicians found these results to be very interesting and a good indicator of instability.

\subsection{Overview of Phase Time Information}

The results shown in Section 6.4 represent individual sit-to-stand transfers. This section focuses on comparing phase time information from data collected from all of the subjects that participated in the clinical trial. The phase time information is calculated for each participant transfer and average transfer times are determined. These phase average times 
are compared to other subjects within the same participant group and a comparison between participant groups is shown as well. Figure 6.23 is a sample plot showing the phase 1 times for 5 young healthy participants. The ${ }^{*}$ 's in the plot represent the phase 1 times for each transfer completed by the subject and the o's represent the average phase 1 time for each subject.

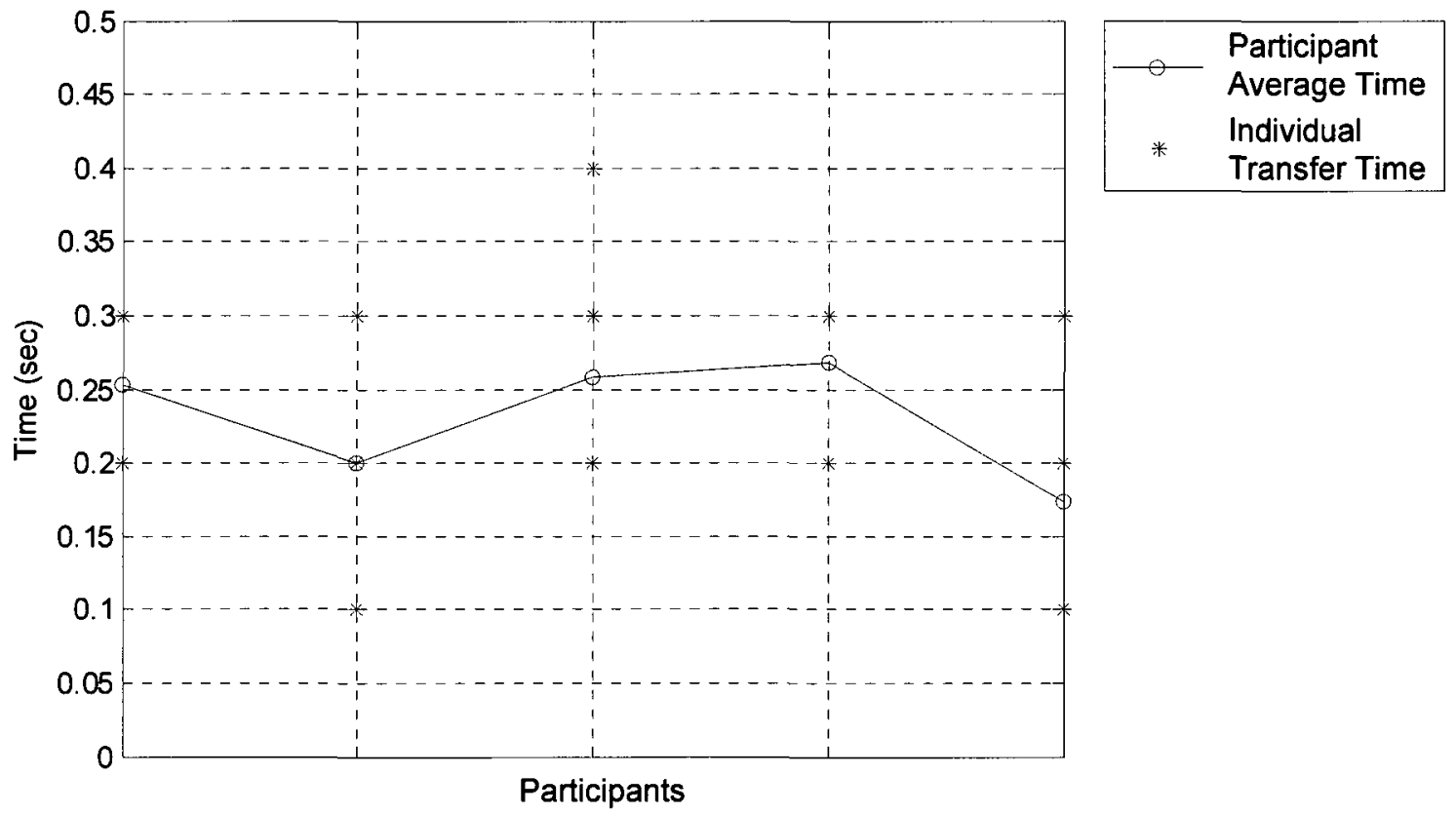

Figure 6.23: Phase 1 transfer times and average times for 5 young healthy participants

Therefore the plot above represents individual participants' phase 1 times $\left(^{*}\right)$ as well as each participants' phase 1 average time (o).

\subsubsection{Clinical Results}

The following plots will show a set of colour coded curves, each representing one participant group. Timing information from individual subjects' phase times and average phase times are plotted along the $\mathrm{x}$-axis. To help show the differences between subjects 
within the same participant group, coloured lines connect the average times for subjects belonging to the same group type.

Figure 6.24 shows the phase 1 times for all four participant groups.

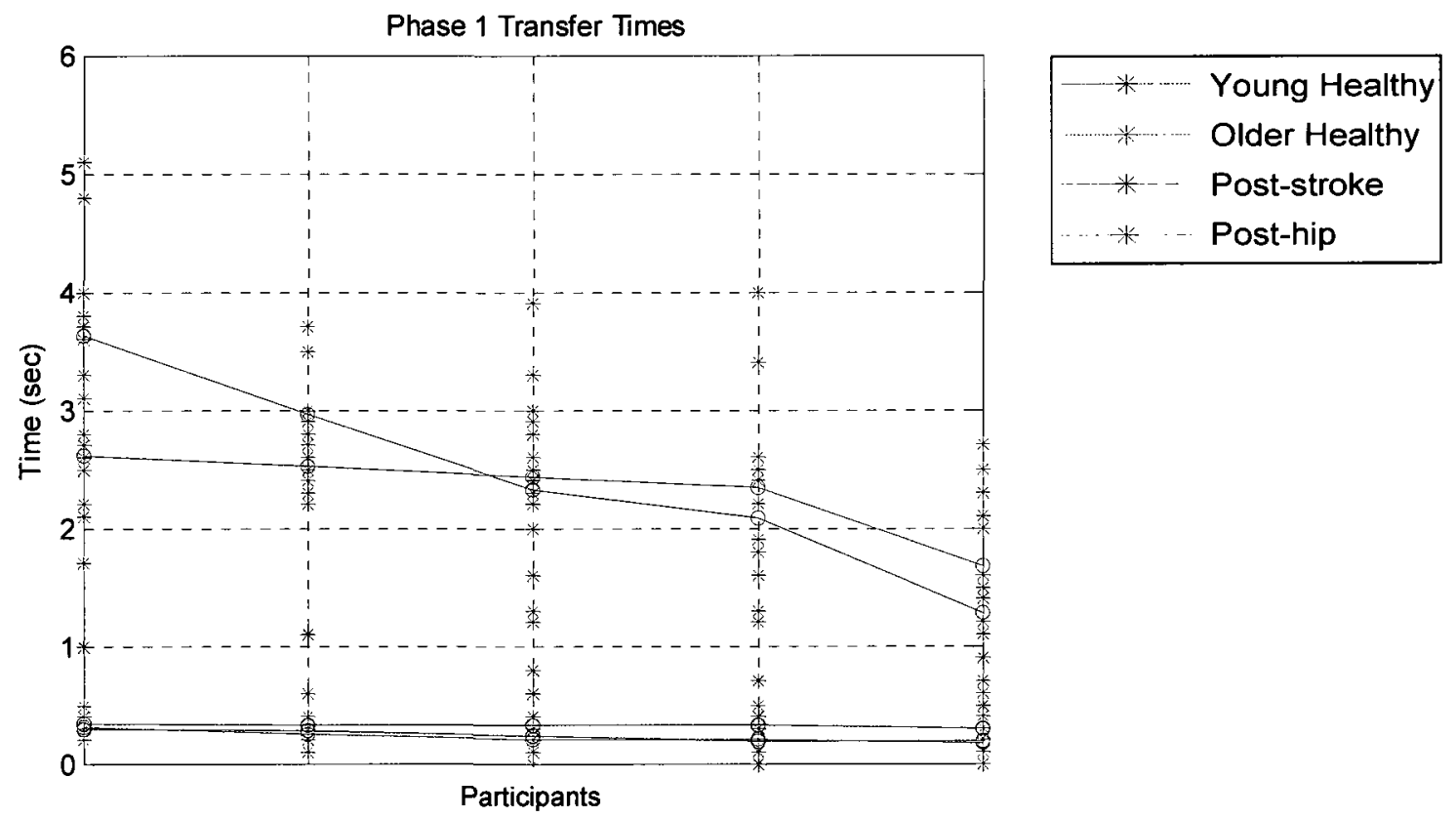

Figure 6.24: Phase 1 times for all four participant groups ( *'s represent individual transfer times and o's represent participant average transfer times)

From the plot it can be seen that the young healthy and older healthy weight shift times are very short compared to the post-stroke and post-hip. There is very little variability between subjects within these two participant groups. On the other hand the post-stroke participant average weight shift times show a great deal of variability ranging from 1.4 seconds to 3.6 seconds approximately. The hip participants' weight shift times do not show much variability between the group but do show some variability within each participant, represented by the large range of *'s. 
Figure 6.25 shows the individual transfer phase 2 times as well as participant average phase 2 times for all four participant groups.

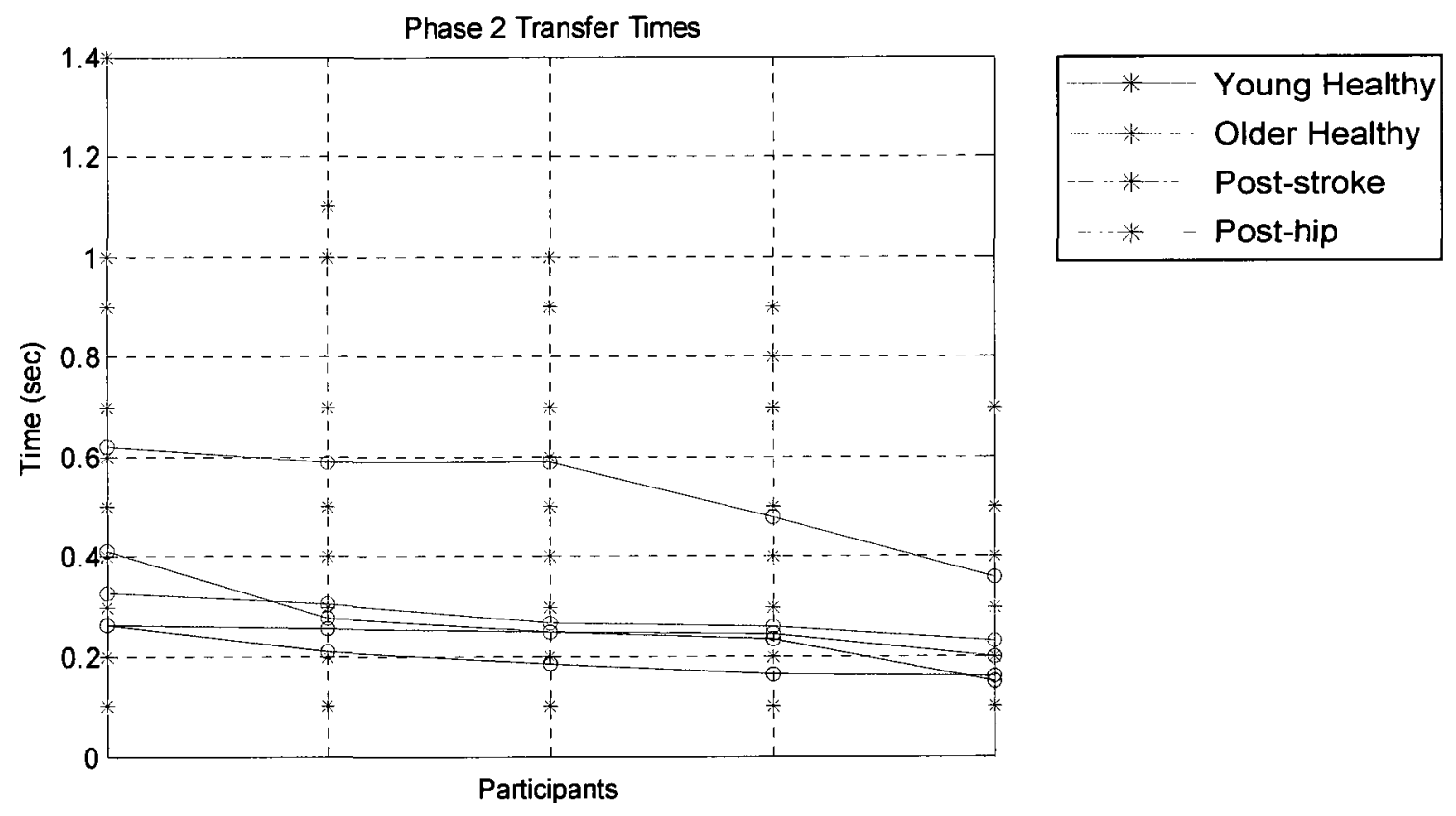

Figure 6.25: Phase 2 times for all four participant groups (*'s represent individual transfer times and o's represent participant average transfer times)

From the plot it can be seen that the young healthy, older healthy and post-stroke participants all have approximately the same transition phase times ranging between 0.2 seconds to 0.4 seconds. The post-hip participants' average transition phase times are greater ranging from 0.38 seconds to 0.6 seconds. The results for the healthy participants are as expected but the difference in the performance of the post-hip and post-stroke participants is very interesting. The transition phase time represents the amount of time a participant spends gradually lifting their weight off of the bed. Analyzing the video data showed that the post-hip participants took the greatest amount of time when transitioning from the weight shift phase to the lift phase, raising their hips very slowly when 
beginning to extend their legs. This is likely due to differences between subject bed exit patterns and differences in movements occurring prior to the transition phase. The posthip participants perform the sit-to-stand sequence very cautiously, particularly during the transition phase, ensuring that they are well balanced before they transfer all of their weight from the bed to their feet. During rehabilitation patients are taught to rise slowly and with great caution to help prevent future falls. The videos and algorithm results showed that the post-hip fracture participants' sit-to-stand sequences were completed more smoothly when compared to the post-stroke participants'. Statistics from the bounce detection for functionally impaired participants algorithm showed that post-hip participants performed fewer shuffles and bounce movements than the post-stroke participants. The post-stroke participants use these extra movements during their weight shift phase to generate momentum, therefore lifting off of the bed very quickly after the weight shift phase and resulting in a short transition phase. The results were verified by the occupational therapy team involved with the study.

Figure 6.26 shows the individual transfer phase 3 times and participant average phase 3 times for all four participant groups. 


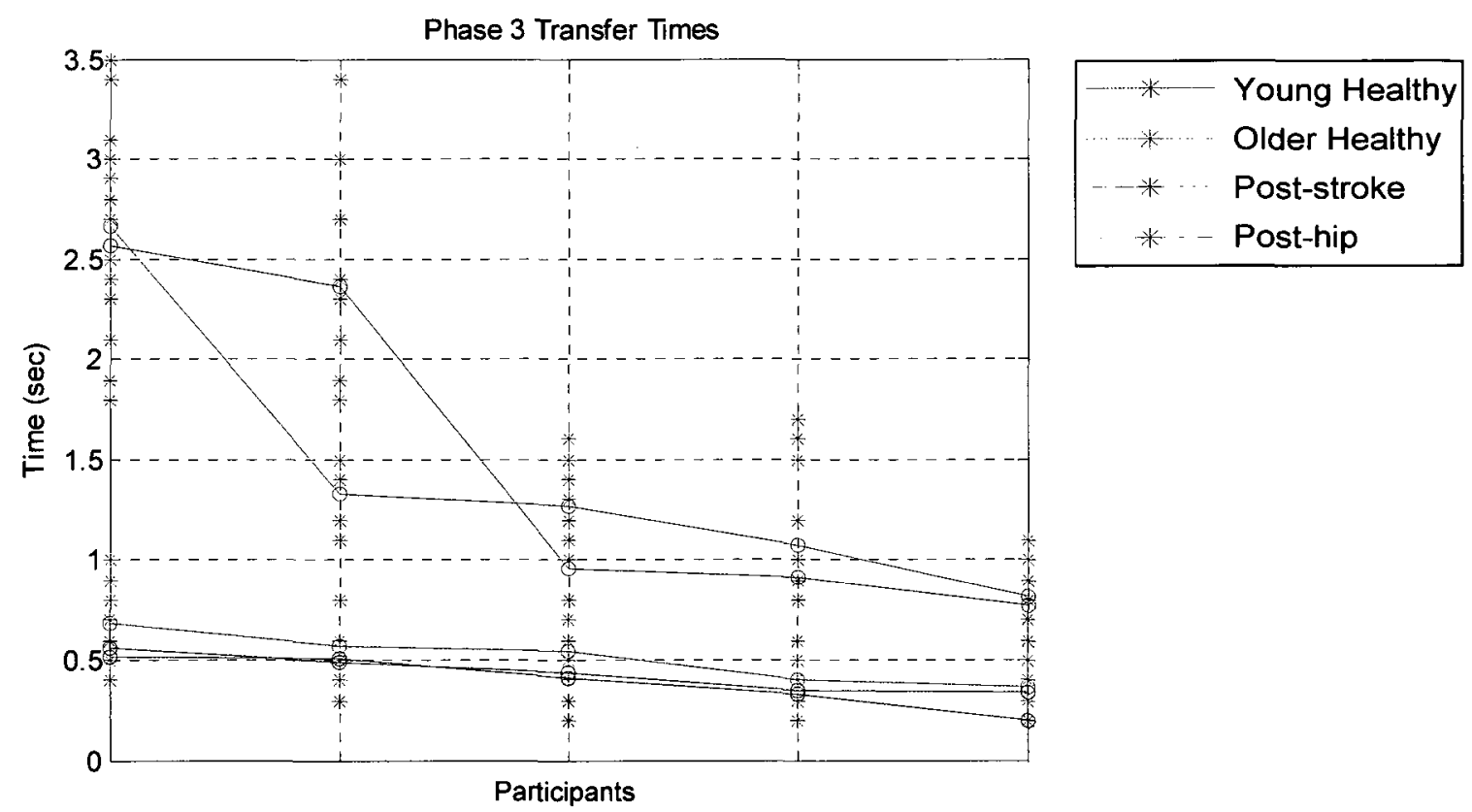

Figure 6.26: Phase 3 times for all four participant groups (*'s represent individual transfer times and o's represent participant average transfer times)

From the results shown above it can be seen that the young healthy participants have the shortest average lift phase times followed closely by the older healthy participants. The variability between participants in each healthy group is minimal. The post-stroke and post-hip participant groups require much longer average lift phase times and show greater variability between participants. This is likely due to the differences between mobility levels within the functionally impaired participant groups.

Figure 6.27 shows the total sit-to-stand times for individual transfers as well as participant average sit-to-stand times for all four participant groups. 


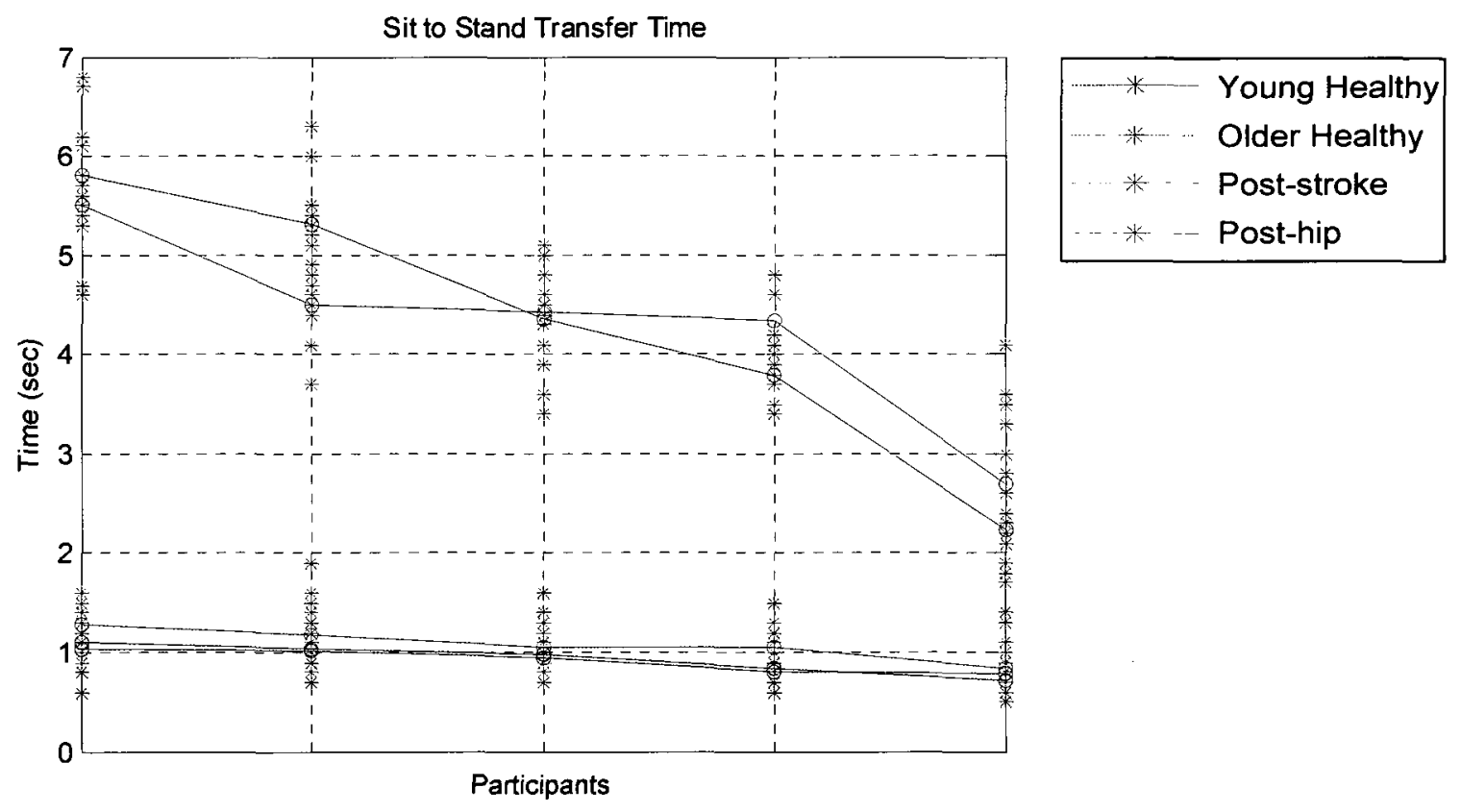

Figure 6.27: Total sit-to-stand times for all four participant groups

(*'s represent individual transfer times and

o's represent participant average transfer times)

From the results shown above it can be seen that the young healthy participants have the fastest sit to stand time followed closely by the older healthy participants. There is very little variability between transfers performed by an individual subject as well as between the subjects within these two groups. The post-hip and post-stroke participants have a much higher sit-to-stand time. The variability between the post-stroke participants' average sit-to-stand times is much greater than the post-hip participants'. These results are to be expected due to the differences in participants recovering from various levels of strokes. 


\subsubsection{Classification Plots}

Scatter plots can be used as another way to show how much the phase times differ for the four participant groups, particularly highlighting the difference between the healthy and functionally impaired groups.

Figure 6.28 shows the dependency between phase 1 and phase 2 timing information for all four participant groups.
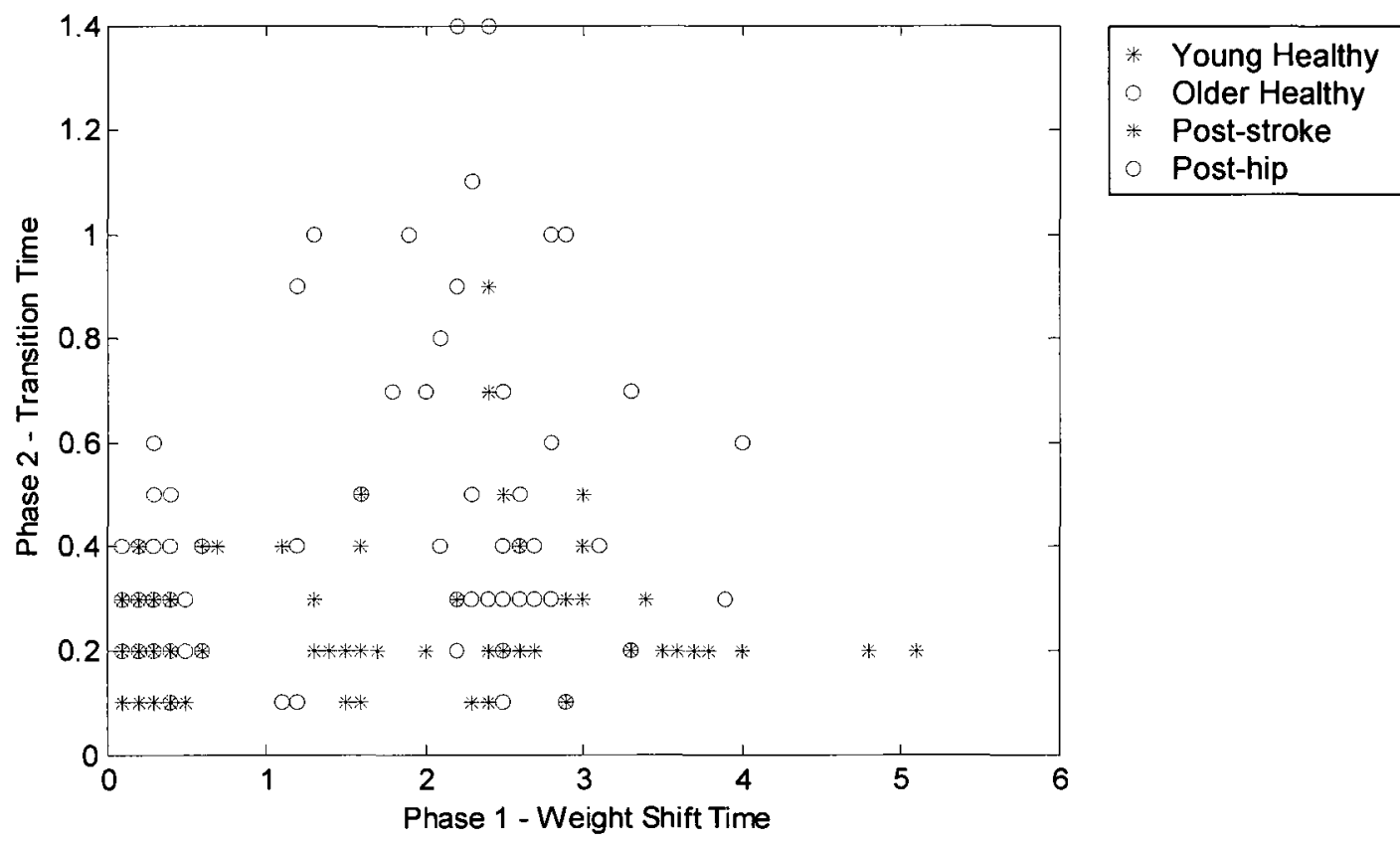

Figure 6.28: Phase 1 vs. Phase 2 classification plot for all four participant groups

From the above plot it is clear that the healthy phase 1 and phase 2 times are extremely small compared to the post-stroke and post-hip times. The post-stroke data is more dependant on the phase 1 times when compared to the post-hip data which is equally scattered in the $\mathrm{x}$ and $\mathrm{y}$ directions.

Figure 6.29 is a plot showing the phase 1 and phase 2 times for the healthy versus the functionally impaired group. 


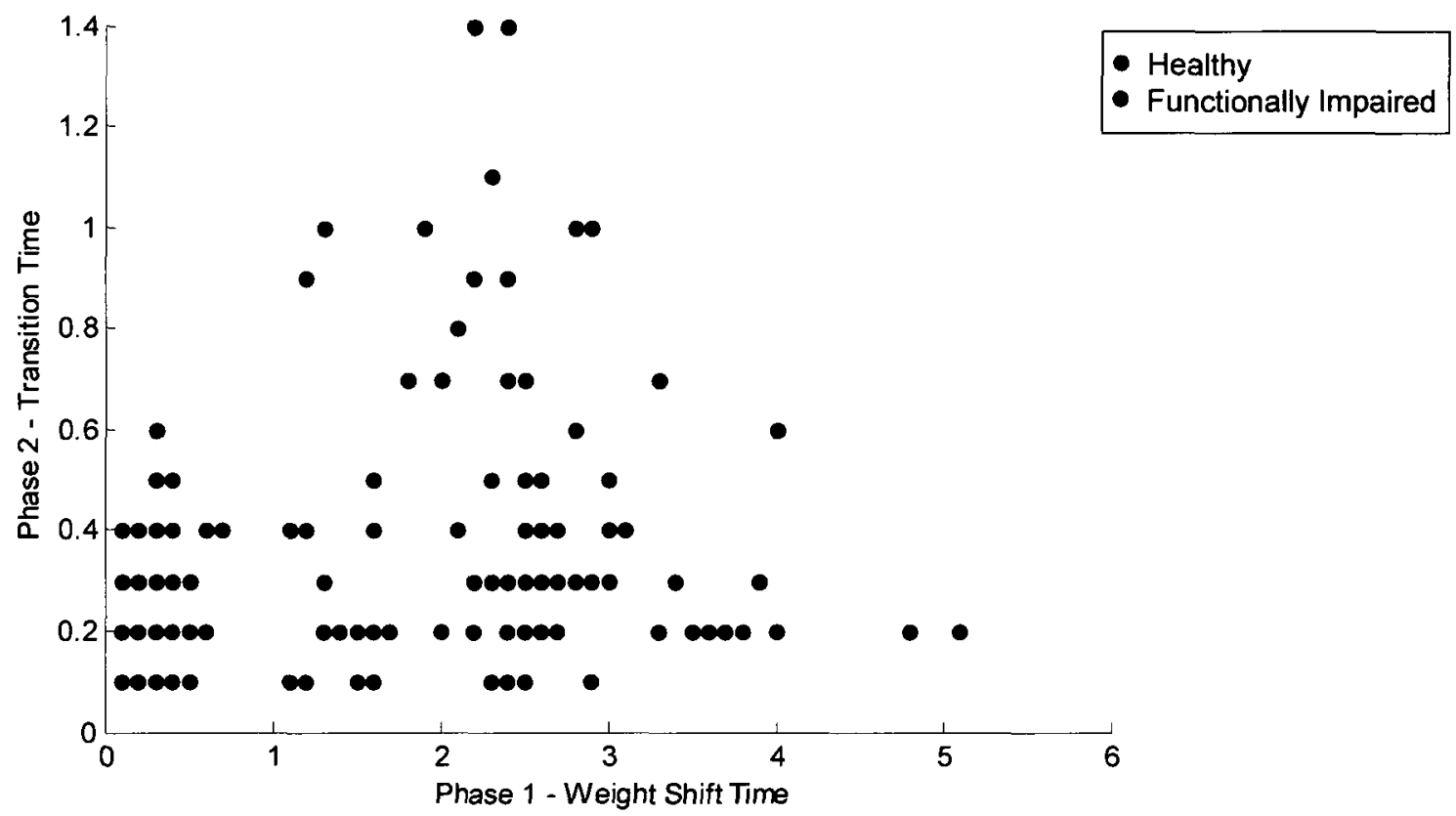

Figure 6.29: Phase 1 vs. Phase 2 classification plot for healthy and functionally impaired groups

From the above plot there is a clear difference between the data results for the healthy group versus the functionally impaired group. The healthy participants' phases 1 and 2 times are negligible when compared to the functionally impaired participants' times.

Figure 6.30 shows the classification plot comparing phase 1 and phase 3 times for all four participant groups. 

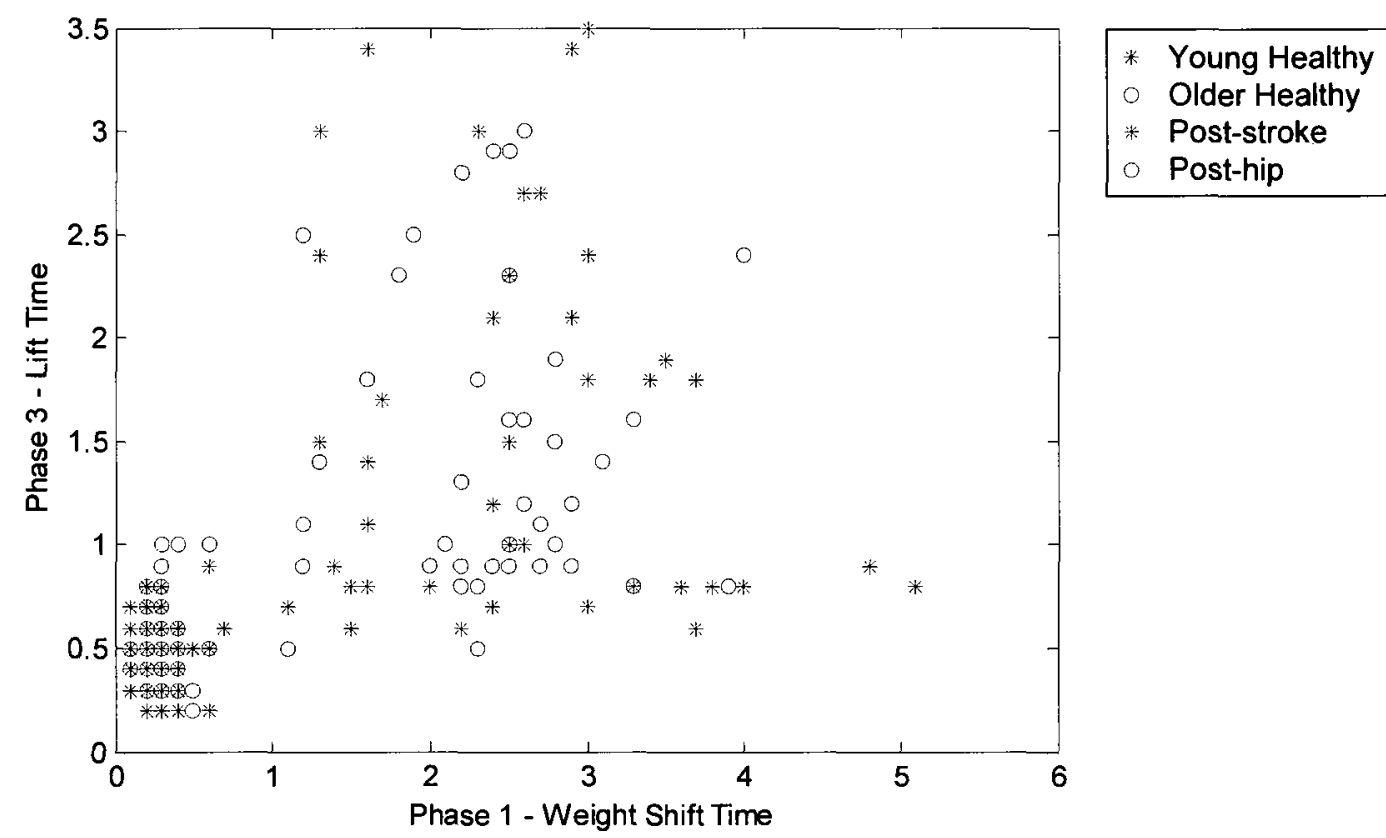

Figure 6.30: Phase 1 vs. Phase 3 classification plot for all four participant groups

The plot above shows that the healthy data is again very separate from the post-stroke and post-hip data results. In this plot there does not appear to be a dependency between the $\mathrm{x}$ and $\mathrm{y}$ direction for any of the four participant groups. The participant timing information is affected by both the phase 1 and phase 3 times equally.

Figure 6.31 shows the phase 1 and phase 3 times for the healthy and functionally impaired groups. 


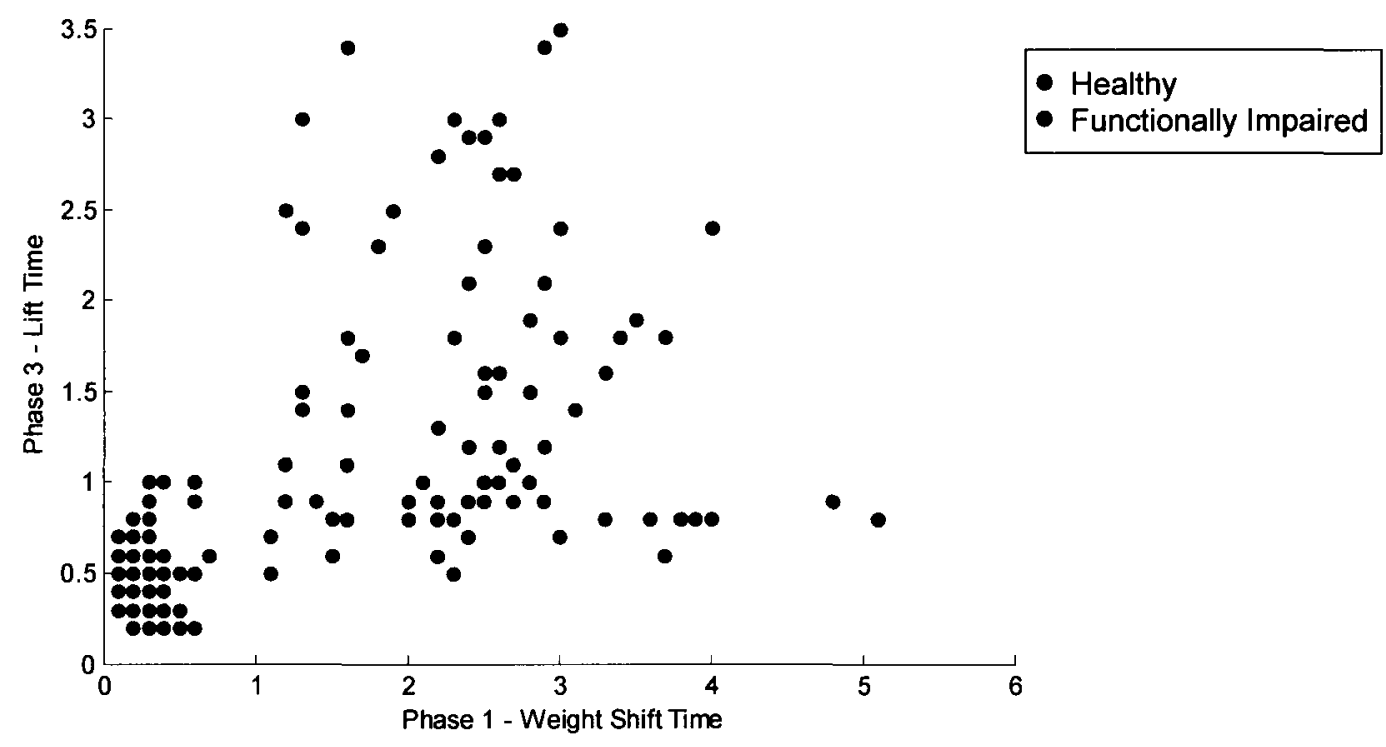

Figure 6.31: Phase 1 vs. Phase 3 classification plot for healthy and functionally impaired groups

The differences between phase 1 and phase 3 times for the healthy and functionally impaired groups is clearly separated. As mentioned above the functionally impaired participant results are equally dependant on both the phase 1 and phase 3 times therefore scattering equally in both the $\mathrm{x}$ and $\mathrm{y}$ direction.

\subsection{Conclusion}

This chapter presented a method for looking at segmenting the sit-to-stand cycle into phases and extracting timing information corresponding to each phase. The method was implemented using a new automated algorithm which looked at separating each sit-tostand sequence into three phases: weight shift, transition and lift. Combining the time from all three phases resulted in the total sit-to-stand time for the individual participant transfer. From the results it was clear that the healthy participants performed their sit-tostand transfers much faster than the functionally impaired participants. The results within 
the healthy participant group did not vary very much from subject to subject. On the other hand there was a great amount of variation between the post-stroke participants and posthip participants. The results showed that phase 1 was the longest phase for both poststroke and post-hip groups and a bounce detection algorithm was presented. The algorithm detected three types of movements: shuffling, bouncing and unsuccessful transfer attempts. The results showed that many participants performed at least one of these movements during the weight shifting phase at the beginning of their sit-to-stand sequence. 


\section{Chapter 7:}

\section{Conclusion}

\subsection{Summary of Results and Contributions}

The thesis research focused on evaluating different methods for analyzing the sit-to-stand pressure data and to differentiate between the pressure patterns produced by the four different adult test groups.

The following is a list of the research contributions that were made:

- Designed and supervised an experiment which collected data from 25 recruited participants at the Élisabeth Bruyère Hospital using a pressure sensitive mat technology.

- Processed the raw sit-to-stand pressure data to create sequences of pressure images and time pressure signals.

- Designed and implemented several region of interest algorithms to detect hip and hand regions from control participant pressure image sequences.

- Designed and implemented an automated algorithm used to segment the clinical trial, participant sit-to-stand pressure signal, into three phases. Performed timing analysis on each phase, identifying the length of each phase as well as the total sit-to-stand time. 
- Designed and implemented an algorithm which performs further analysis on the functionally impaired participants' pressure signals to detect additional movements such as shuffling, bouncing and unsuccessful transfers.

\subsection{Recommendations for Future Work}

The following is a list of recommendations for future work:

- Repeat the clinical trial, collecting data from all 25 participants again particularly the older healthy and post-stroke and post-hip groups. Perform analysis on the new pressure data to help identify changes in patients' movements. The results could help evaluate whether the health and mobility of the participants has increased or decreased.

- Design a region of interest extraction algorithm to extract hip and hand information from the pressure images produced using the participant pressure data collected during the clinical trial, in addition to the control participant data that was presented in this thesis.

- Design an algorithm for evaluating patient symmetry during individual sit-tostand transfer sequences.

- Synchronize the foot pressure data with the bed pressure data collected. Use the additional foot data to add a fourth phase when segmenting the sit-to-stand sequence. The foot data would allow for the addition of a stabilization phase.

- Combine and implement the various algorithms into the smart home to monitor people and detect changes in mobility. 


\section{List of References:}

[1] Health Canada Division of Aging and Seniors, "Canada's Aging Population." 2002. http://www.phac-aspc.gc.ca/seniors-aines/pubs/fed_paper/pdfs/fedpager e.pdf.

Last accessed: June 10, 2009.

[2] K. Cook, "Frozen in time: life in the face of chronic care cutbacks," CMAJ, pp. 1045-1047, April 1999.

[3] F. Knoefel, Ontario Ministry of Health and Long Term Care, private communication, 2009.

[4] A. Arcelus, M. Jones, R. Goubran, F. Knoefel, "Integration of Smart Home Technologies in a Health Monitoring System for the Elderly," in Proc., International Conference on Advanced Information Networking and Applications Workshop, ON, Canada, May 2007, pp 820-825.

[5] X. Le, M. Mascolo, A. Gouin, N. Noury, "Health Smart Home - Towards an assistant tool for automatic assessment of the dependence of elders," in Proc., IEEE International Conference of the Engineering in Medicine and Biology Society, Lyon, France, Aug 2007, pp. 3806-3809.

[6] N. Noury, "AILISA: experimental platforms to evaluate remote care and assistive technologies in gerontology," in Proc., International Workshop on Enterprise Networking and Computing in Healthcare Industry, Busan, Korea, June 2005, pp. $67-72$.

[7] X. Le, M. Mascola, A. Gouin, N. Noury, "Health Smart Home for elders - A tool for automatic recognition of activities of daily living," in Proc., IEEE International Conference of the Engineering in Medicine and Biology Society, BC, Canada, Aug 2008, pp. 3316-3319.

[8] J. Poujaud, N. Noury, "Identification of inactivity behaviour in Smart Home," in Proc., IEEE International Conference of the Engineering in Medicine and Biology Society, BC, Canada, Aug 2008, pp. 2075-2078.

[9] A. Fleury, N. Noury, M. Vacher, H. Glasson, J.-F. Serignat, "Sound and Speech Detection and Classification in a Health Smart Home," in Proc., IEEE International Conference of the Engineering in Medicine and Biology Society, BC, Canada, Aug 2008 , pp. 4644-4647. 
[10] N. Noury, G. Virone, P. Barralon, J. Ye, V. Rialle, J. Demongeot, "New Trends in Health Smart Homes," in Proc., International Workshop on Enterprise Networking and Computing in Healthcare Industry, CA, USA, June 2003, pp. 118-127.

[11] M. Ogawa, T. Togawa, "Attempts at monitoring health status in the home," in Proc., International Conference on Microtechnolgies in Medicine and Biology, Lyon, France, Oct 2000, pp. 552-556.

[12] P. Bartolomeu, J. Fonseca, F. Vasques, "Challenges in Health Smart Homes," in Proc., International Conference on Pervasive Computing Technologies for Healthcare, Tampere, Finland, Jan 2008, pp. 19-22.

[13] L. Saint-Bauzel, V. Pasqui, G. Morel, B. Gas, "Real-time human posture observation from a small number of joint measurements," in Proc., IEEE International Conference on Intelligent Robots and Systems, CA, USA, Oct 2007, pp. 3956-3961.

[14] M. Jones, A. Arcelus, R. Goubran, F. Knoefel, "A Pressure Sensitive Home Environment," in Proc., IEEE International Workshop on Haptic Audio Visual Environments and their Applications, ON, Canada, Nov 2006, pp. 820-825.

[15] A. Gaddam, S. Mukhopadkyay, S. Gupta, "Integrating a Bed Sensor in a Smart Home Monitoring System," in Proc., IEEE International Instrumentation and Measurement Technology Conference, BC, Canada, May 2008, pp. 518-521.

[16] M. Ermes, J. Parkka, J. Mantyjarvi, I. Korhonen, "Detection of Daily Activities and Sports Wearable Sensors in Controlled and Uncontrolled Conditions," IEEE Trans., Information Technology in Biomedicine, vol. 12, pp. 20-26, Jan 2008.

[17] B. Najafi, K. Aminian, A. Paraschiv-Ionescu, F. Loew. C. Bula, P. Robert, "Ambulatory System for Human Motion Analysis Using a Kinematic Sensor: Monitoring of Daily Physical Activity in the Elderly," IEEE Trans., Biomedical Engineering, vol. 50, pp. 711-723, June 2003.

[18] A. Salarian, H. Russmann, F. Vingerhoets, P. Burkhard, K. Aminian, "Ambulatory Monitoring of Physical Activities in Patients With Parkinson's Disease," IEEE Trans., Biomedical Engineering, vol. 54, pp. 2296-2299, Dec 2007.

[19] P. Barralon, N. Noury, N. Vuillerme, "Classification of Daily Physical Activities from a Single Kinematic Sensor," in Proc., IEEE International Conference of the Engineering in Medicine and Biology Society, Shanghai, China, Sept 2005, pp. 2447-2450.

[20] H. Knight, J. Lee, H. Ma, "Chair Alarm for Patient Fall Prevention based on Gesture Recognition and Interactivity," in Proc., IEEE International Conference of the Engineering in Medicine and Biology Society, BC, Canada, Aug 2008, pp. 3698-3701. 
[21] K. Kamiya, M. Kudo, H. Nonaka, J. Toyama, "Sitting Posture Analysis by Pressure Sensors," in Proc., International Conference on Pattern Recognition, FL, USA, Dec 2008, pp. 1-4.

[22] M. Yamada, M. Kudo, H. Nonaka, J. Toyama, "Hipprint Person Identification and Behavior Analysis," in Proc., International Conference on Pattern Recognition, Hong Kong, China, Aug, 2006, pp. 533-536.

[23] T. Harada, T. Sato, T. Mori, "Estimation of Bed-Ridden Human's Gross and Slight Movement Based on Pressure Sensors Distribution Bed," in Proc., IEEE International Robotics \& Automation Conference, DC, USA, May 2002, pp. 37953800 .

[24] A. Kalamdani, C. Messon, M. Siegel, "Tactile Sensing by the Sole of the Foot: Part I: Apparatus and Initial Experiments Toward Obtaining Dynamic Pressure Maps Useful for Stabilizing Standing, Walking, and Running of Humanoid Robots," in Proc., IEEE International Workshop on Haptic Audio Visual Environments and their Applications, ON, Canada, Nov 2006, pp. 147-151.

[25] R. Aissaoui, J. Dansereau, "Biomechanical Analysis and Modelling of Sit to Stand Tasks: A Literature Review," in Proc., IEEE International Conference on Systems, Man, and Cybernetics, Tokyo, Japan, 1999, pp. 141-146.

[26] G. Gehlsen, M. Whaley, "Falls in the elderly: Part II, Balance, strength, and flexibility," Arch. Phys. Med. Rehabil., pp. 739-741, 1990.

[27] J. McChesney, "Balance testing benefits older adult athletes," Biomechanics, pp. 29-35, 2002.

[28] P. Overstall, A. Exton-Smith, F. Imms, A. Johnson, "Falls in the elderly related to postural imbalance," Br. Med. J., pp. 261-264, 1977.

[29] C. Ring, U. Nayak, B. Isaac, "Balance and function in elderly people who have and who have not fallen," Arch. Phys. Med. Rehabil., pp. 261-264, 1988.

[30] W. Janssen, H. Bussmann, H. Stam, "Determinants of the Sit-to-Stand Movement: A Review," Physical Therapy, vol. 82, pp. 866-879, Sept 2002.

[31] N. Alexander, D. Koester, J. Grunawalt, "Chair design affects how older adults rise from a chair," J. American Geriatrics Society, vol. 44, pp. 356-362, 1996.

[32] B. Etnyre, D. Thomas, "Event Standardization of Sit-to-Stand Movements," Physical Therapy, vol. 87, pp. 1651-1666, Dec 2007.

[33] K. Kerr, J. White, D. Barr, R. Mollan, "Analysis of the sit-stand-sit movement cycle in normal subjects," Clinical Biomechanics, vol. 12, pp. 236-245, 1997. 
[34] U. Lindemann, R. Muche, M. Stuber, W. Zijlstra, K. Hauer, C. Becker, "Strength Exertion During the Chair-Rise Movement in Very Old People," J. Gerontology., vol. 62, pp. 636-640, June 2007.

[35] J. Feland, R. Hager, R. Merrill, "Sit to stand transfer: performance in rising power, transfer time and sway by age and sex in senior athletes," British Journal of Sports Medicine, March 2005.

[36] M. Lomaglio, J. Eng, "Muscle strength and weight-bearing symmetry relate to sitto-stand performance in individuals with stroke," Gait \& Posture, vol. 22, pp. 126$131,2005$.

[37] C. Duclos, S. Nadeau, J. Lecours, "Lateral Trunk Displacement and Stability During Sit-to-Stand Transfer in Relation to Foot Placement in Patients With Hemiparesis," Neurorehabil Neural Repair, vol. 22, pp. 715-722, Nov 2008.

[38] B. Najafi, K. Aminiam, F. Loew, Y. Blanc, P. Robert, "Measurement of Stand-Sit and Sit-Stand Transitions Using a Miniature Gyroscope and Its Application in Fall Risk Evaluation in the Elderly," Biomedical Engineering, IEEE Transactions on, vol. 49, pp. 843-851, Aug 2002.

[39] P. Cheng, M. Liaw, M. Wong, F. Tang, M. Lee, P. Lin, "The Sit-to-Stand Movement in Stroke Patients and Its Correlation With Falling," Arch. Phys. Med. Reahabil., vol. 79, pp. 1043-1046, Sept 1998.

[40] T. Yamada, S. Demura, "Relationships between ground reaction force parameters during a sit-to-stand movement and physical activity and falling risk of the elderly and a comparison of the movement characteristics between the young and the elderly," Archives of Gerontology and Geriatrics, vol. 48, pp. 73-77, 2009.

[41] C. Ford-Smith, A. VanSant, "Age Differences in Movement Patterns Used to Rise from a Bed in Subjects in the Third Through Fifth Decades of Age,", Physical Therapy, vol. 73, pp. 300-309, May 1993.

[42] M. Galli, V. Cimolin, M. Crivellini, I. Campanini, "Quantitative analysis of sit to stand movement: Experimental set-up definition and application to healthy and hemiplegic adults," Gait \& Posture, vol. 28, pp. 80-85, Oct 2007.

[43] S. Allin, A. Mihailidis, "Low-cost, Automated Assessment of Sit-To-Stand Movement in "Natural" Environments," in Proc., International Federation for Medical and Biological Engineering Conference, BC, Canada, Aug 2008, vol. 22, pp. 76-79.

[44] M. Goffredo, M. Schmid, S. Conforto, M. Carli, A. Neri, T. D’Alessio, "Markerless Human Motion Analysis in Gauss-Laguerre Transform Domain: An Application to Sit-To-Stand in Young and Elderly People," IEEE Trans., Information Technology in Biomedicine, vol. 13, pp. 207-216, March 2009. 
[45] D. Rosenbaum H. Becker, "Plantar pressure distribution measurements. Technical background and clinical applications," Foot and Ankle Surgery, vol. 3, pp. 1-14, Mar 1997.

[46] S. Yao, C. Asawa, "Fiber Optical Intensity Sensors," IEEE Trans., Journal on Selected Areas in Communications, vol. 3, pp. 562-575, Apr 1983.

[47] Y. Zhu, A. Wang, "Miniature Fiber-Optic Pressure Sensor," IEEE Photonics Technology Letters, vol. 17, pp. 447-449, Feb 2005.

[48] E. Cibula, D. Donlagic, C. Stropnik, "Miniature Fiber Optic Pressure Sensor for Medical Applications," in Proc., IEEE Sensors, FL, USA, June 2002, pp. 711-714.

[49] G. Krishna, K. Rajanna, "Tactile Sensor Based on Piezoelectric Resonance," IEEE Trans., Sensors Journal, vol. 4, pp. 691-697, Oct 2004.

[50] C. Lebosse, B. Bayle, M. Mathelin, P. Renaud, "Nonlinear modeling of low cost force sensors," in Proc., IEEE Conference on Robotics and Automation, CA, USA, May 2008, pp. 3437-3442.

[51] E. Reimer, L. Baldwin, "Cavity sensor technology for low cost automotive safety and control devices," http://www.canpolar.com/principles.shtm, 1999. Last accessed July 25, 2009.

[52] H. Morishita, R. Fukui, T. Sato, "High resolution pressure sensor distributed floor for future human-robot symbiosis environments," in Proc., IEEE/RSJ International Conference on Intelligent Robots and Systems, Lausanne, Switzerland, Oct 2002, pp. 1246-1251.

[53] R. Taylor, P. Jensen, L. Whitcomb, A. Barnes, R. Kumar, D. Stoianovici, P. Gupta, Z. Wang, E. deJuan, L. Kavoussi, "A Steady-Hand Robotic System for Microsurgical Augmentation," in Proc., International Conference on Medical Image Computing and Computer-Assisted Intervention, Cambridge, England, Sept 1999, pp. 1031-1041.

[54] W. Lie, "Automatic Target Segmentation by Locally Adaptive Image Thresholding," IEEE Trans., Image Processing, vol. 4, pp. 1036-1041, July 1995.

[55] X. Zhang, M. Desai, "Segmentation of Bright Targets Using Wavelets and Adaptive Thresholding," IEEE Trans., Image Processing, vol. 10, pp. 1020-1030, July 2001.

[56] A. Bovik, "Basic Binary Image Processing," in Handbook of Image and Video Processing, $2^{\text {nd }}$ ed., Burlington, Mass.: Elsevier Academic Press, 2005, pp. 39-44.

[57] J. Shim, C. Dorai, "A Generalized Region Labeling Algorithm for Image Coding, Restoration, and Segmentation," in Proc., International Conference on Image Processing, Kobe, Japan, Oct 1999, pp. 46-50. 
[58] R. Dufour, E. Miller, N. Galatsanos, "Template Matching Based Object Recognition With Unknown Geometric Parameters," IEEE Trans., Image Processing, vol. 11, pp. 1385-1396, Dec 2002.

[59] O. Bellon, L. Silva, "New Improvements to Range Image Segmentation by Edge Detection," IEEE Signal Processing Letters, vol. 9, pp. 43-45, Feb 2002.

[60] Tactex Controls Inc., "Array Sensors," http://www.tactex.com/large arrays.php, 2007. Last accessed June 5, 2009.

[61] A. Arcelus, M. Holtzman, I. Veledar, R. Goubran, H. Sveistrup, and P. Guitard, "Contact location estimation from a nonlinear array of pressure sensors," in Proc., IEEE Instrumentation and Measurement Technology Conference, Victoria, BC, Canada, May 2008, pp 1969-1973.

[62] M. Holtzman, A. Arcelus, I. Veledar, R. Goubran, H. Sveistrup, and P. Guitard, "Force Estimation with a Non-Uniform Pressure Sensor Array," in Proc., IEEE Instrumentation and Measurement Technology Conference, Victoria, BC, Canada, May 2008, pp. 1974-1979.

[63] The MathWorks Inc., "Image Processing Toolbox", http://www.mathworks.com/access/helpdesk/help/toolbox/images,

1984-2009. Last accessed June 15, 2009.

[64] J. Manikandan, B. Venkataramani, M. Jayachandran, "Evaluation of Edge Detection Techniques towards Implementation of Automatic Target Recognition," in Proc., International Conference on Computational Intelligence and Multimedia Applications, Tamil Nadu, India, Dec 2007, pp. 441-445.

[65] P. Mlsna, J. Rodriguez, "Gradient and Laplacian Edge Detection," in Handbook of Image and Video Processing, $2^{\text {nd }}$ ed., Burlington, Mass.: Elsevier Academic Press, 2005, pp. 535-548.

[66] Y. Luo, R. Duraiswami, "Canny Edge Detection on NVIDIA CUDA," in Proc., Computer Vision and Pattern Recognition Workshops, AK, USA, June 2008, pp. 1-8.

[67] S. Nuzik, R. Lamb, A. VanSant, S. Hirt, "Sit-to-Stand Movement Pattern -A Kinematic Study," Physical Therapy, vol. 66, pp. 1708-1713, 1986.

[68] M. Schenkman, R. Berger, P. Riley, R. Mann, A. Hodge, "Whole-Body Movements During Rising to Standing from Sitting," Physical Therapy, vol. 70, pp. 638-651, 1990.

[69] P. Riley, M. Schenkman, R. Mann, W. Hodge, "Mechanics of a constrained chairrise," Journal of Biomechanics, vol. 24, pp. 77-85, 1991. 
[70] P. Millington, B. Myklebust, G. Shambes, "Biomechanical analysis of the sit-tostand motion in elderly persons," Arch. Phys. Med. Rehabil., vol. 73, pp. 609-617, 1992.

[71] K. Miyoshi, T. Kimura, Y. Yokokawa, G. Chen, T. Fujiwara, I. Yamamoto, Y. Kondo, "Effect of Ageing on Quadriceps Muscle Strength and on the Forward Shift of Center of Pressure during Sit-to-stand Movement from a Chair," Journal of Phys. Ther. Sci., vol. 17, pp. 23-28, 2005.

[72] A. Arcelus, C. Herry, R. Goubran, F. Knoefel, H. Sveistrup, M. Bilodeau, "Determination of sit-to-stand transfer duration using bed and floor pressure sequences," IEEE Trans., Biomedical Engineering, to be published in 2009. 


\section{Appendix A:}

\section{Equipment Setup User Manual}

\section{A.1 Hardware Setup}

\section{A.1.1 Three Bed Mat Configuration}

The three large pressure sensor arrays (bed mats) should be placed side by side below the mattress as shown in the diagram below.

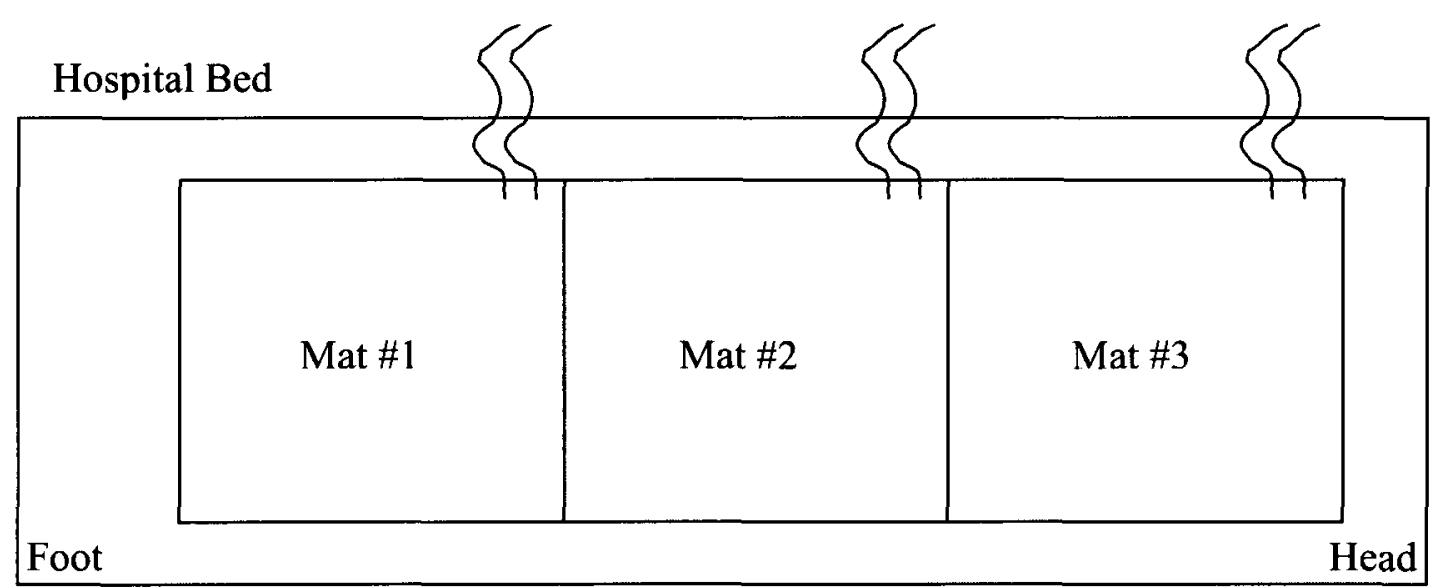

\section{A.1.2 Individual Bed Mat to Computer Connection}

1. Connect each RJ12 bed mat cable to a RJ12-RJ45 converter box.

2. Connect each RJ45 cable to the STL-232 interface.

3. Connect each $9 \mathrm{~V}$ power supply to the STL-232 interface.

4. Connect each STL-232 interface to the NPort using a serial to RJ45 cable.

5. Connect the NPort power cord to the wall.

6. Connect the NPort to the computer using a crossover Ethernet cable. 


\section{A.1.3 Floor Mat Configuration}

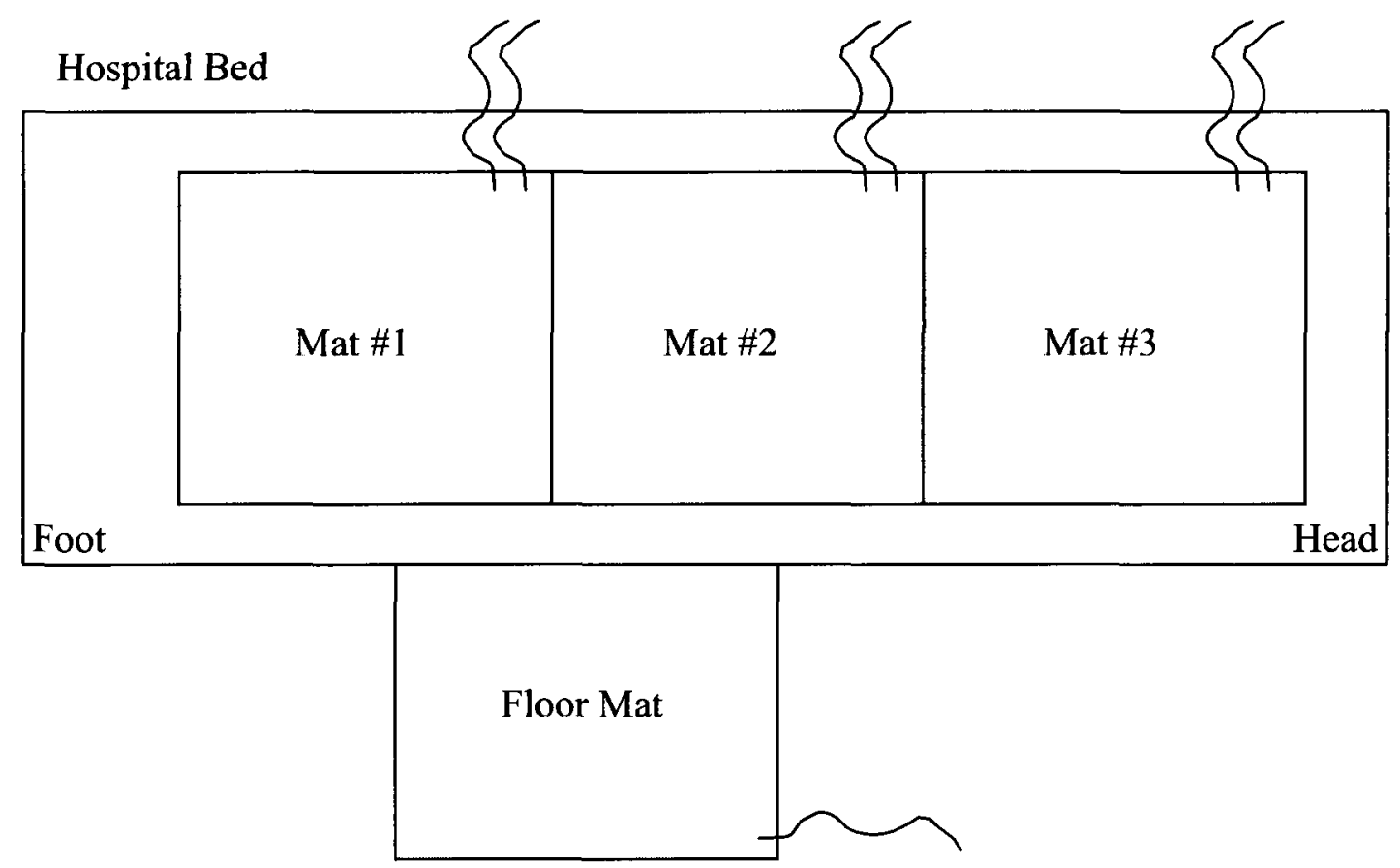

\section{A.1.4 Floor Mat to Computer Connection}

1. Connect the floor mat cable to the power supply.

2. Connect the power supply to the wall outlet using the power cable.

3. Connect the power supply to the computer using the USB cable. 


\section{A.2 Software Setup}

\section{A.1.5 Computer Setup}

1. Set the computer IP address to 192.168.127.250.

i. Click on 'Start', go to 'Setting', and select 'Network Connections'.

ii. From the list of connections click on 'Local Area Connection'.

iii. A 'Local Area Connection Status' window should pop up, select 'Properties'.

iv. From the 'Properties' window scroll to select 'Internet Protocol' and click 'Properties'.

v. Note the current configuration, if an IP address is displayed, otherwise click 'Use the following IP address'.

vi. Set the IP address field to 192.168.127.250.

vii. Set the Subnet mask to a default: 255.255.255.0.

viii. Click 'OK' and in the Network Connections window ensure the 'Details' of the Local Area Connection has changed the IP Address.

2. Install the drivers using the provided software from Tactex.

3. Copy the folder "Mobility Project" from the provided "Mobility Project" CD containing the following files/programs.

i. TTXMIO Sensor Demo.EXE

ii. 6 sensors.txt

iii. default.daq

iv. clxio.dll

v. normal.txt

vi. Mobility Sensor Tile.exe

vii. HDSensor TileMap-4.txt

viii. usbcan32.dll

ix. vcand32.dll

x. ttxcmnwin.dll 
xi. ttxsensortile.dll

xii. demo.ini

\section{A.1.6 NPort (black box) Setup}

1. Modifying firmware.

I. Set ports \#1-6 to "Real COM Mode".

II. Disable ports \#7 and \#8.

III. IP address should be set to default: 192.168.127.25. 


\section{Appendix B:}

\section{Data Acquisition User Manual}

\section{A.3 Initializing Connection to Bed Mats}

1. Select the "NPort Administrator" shortcut from the Desktop.

A window should open which reads "NPort Administrator-Configuration" at the top. The window is divided into 3 panels, the "Function" panel to the left, a "Configuration" panel, and a "Log" panel at the bottom. This is shown in Figure B.1 below.

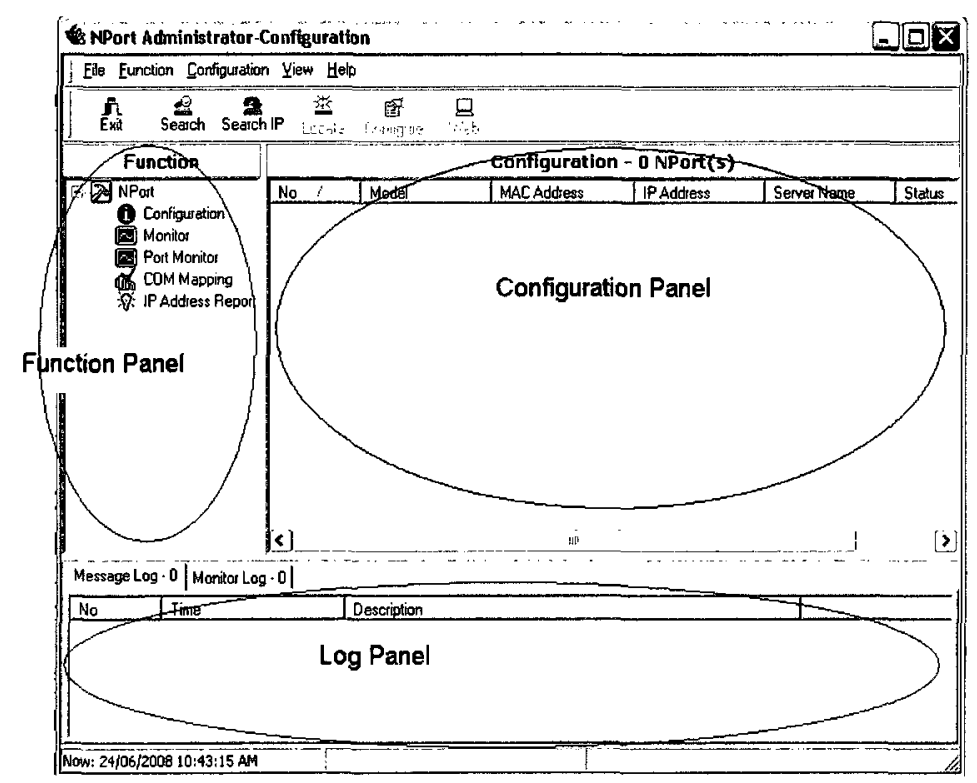

Figure B.1: NPort Administrator View

2. Make sure that within the "Function" panel, "Configuration" is highlighted, as in the figure above.

3. Now, select "Search" from the tool bar. A "Searching" window should appear 
and one NPort will be listed as shown in Figure B.2 below.

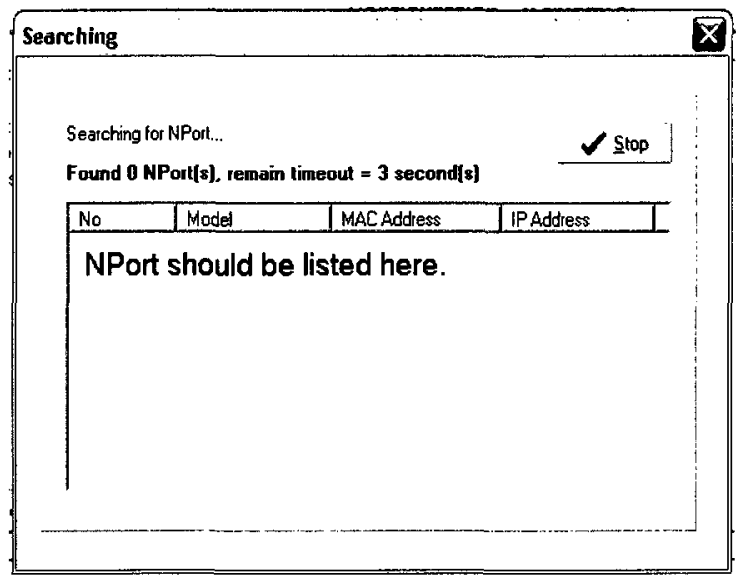

Figure B.2: Selecting the NPort

Note: If the NPort does not appear in this list you will not be able to connect, see Troubleshooting (Section B.4) within this user manual.

4. In the "Searching" window, select NPort with IP address 192.168.127.254.

5. Click on COM Mapping in the "Function" panel.

If the Configuration panel already contains a list of COM Ports with the correct IP Address, as in Figure B.3 below, then follow a) otherwise if the Configuration panel is blank follow the instructions in b).

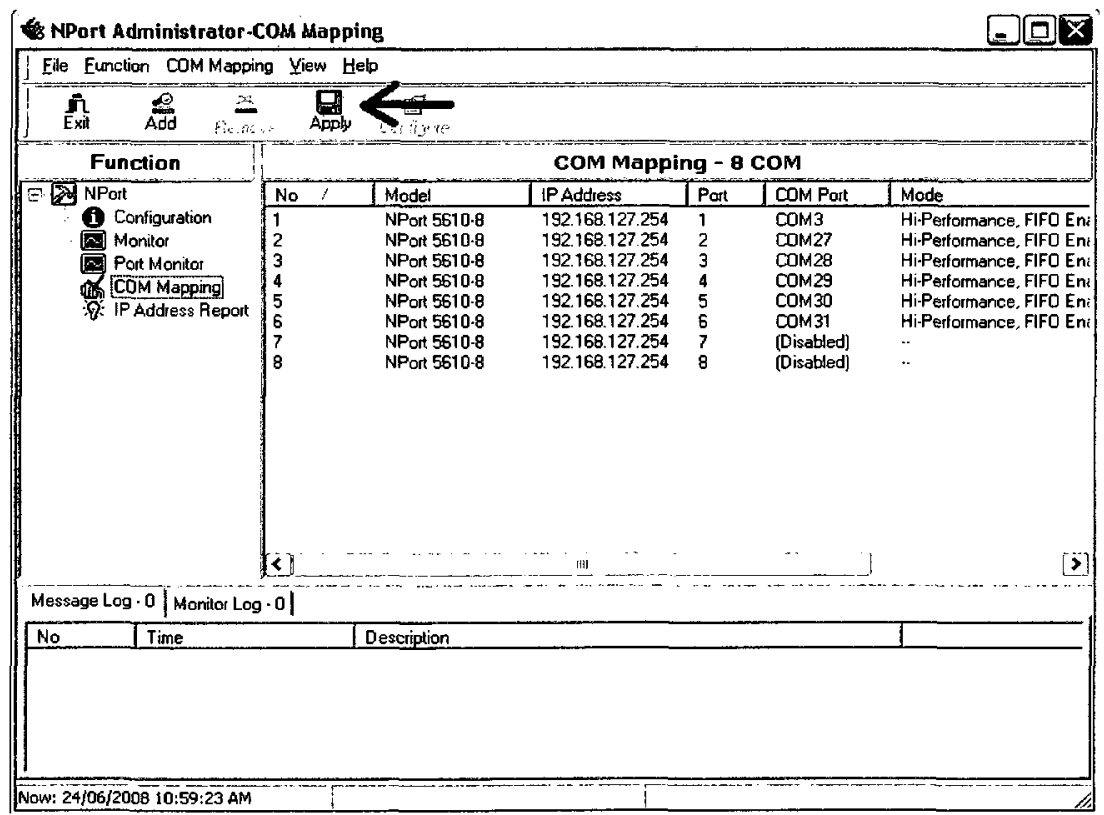

Figure B.3: COM Port Mapping 
a) Click "Apply" in the tool bar.

b) Otherwise follow the steps below.

i. Select COM Mapping $\rightarrow$ Import COM Mapping (from the menu bar).

ii. Click Browse...

iii. Select COM port mapping.

iv. Click Open.

v. Click OK.

vi. Click OK.

vii. Select Apply from the tool bar.

6. Click Yes.

7. Click OK.

8. Close the NPort Administrator.

These steps initialize a pathway between the 6 bed mats and the computer. The next step will now establish the connection between the bed mats and the computer, as well as the floor mat and the computer.

\section{A.4 Floor Mat and Bed Mat Connections}

1. On the desktop there should be a folder named "Floor", open this folder.

2. Scroll down and select "Run Mobility Sensor Tile.exe". A window as shown in Figure B.4 below will appear.

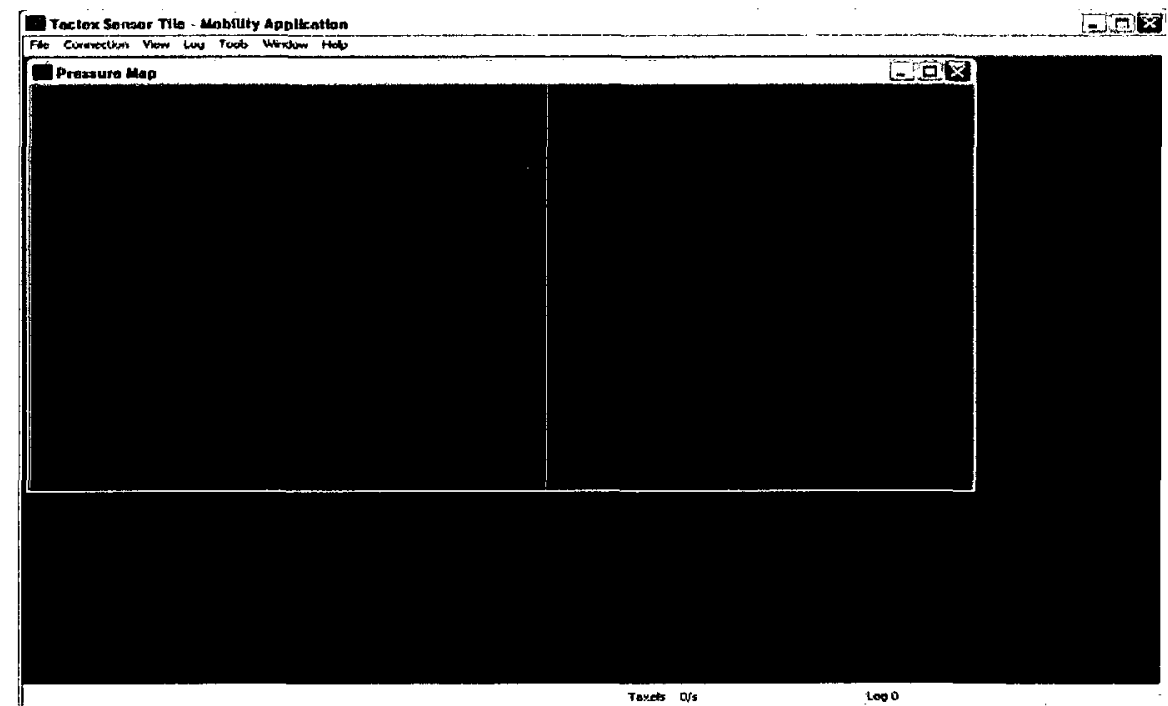

Figure B.4: Floor Sensor Data Acquisition Software 
3. Select Connection $\rightarrow$ Start Data Stream.

4. Click OK on "Starting raw data" dialog box.

5. Select Tools $\rightarrow$ Tare Sensors.

NOTE: make sure that nothing is on the floor mat when you select "Tare Sensors".

6. Now run the Bed Mat data acquisition software, by selecting "TTXMIO Sensor Demo.exe" from the BED folder located on the desktop. A window, Figure B.5 shown below, will appear.

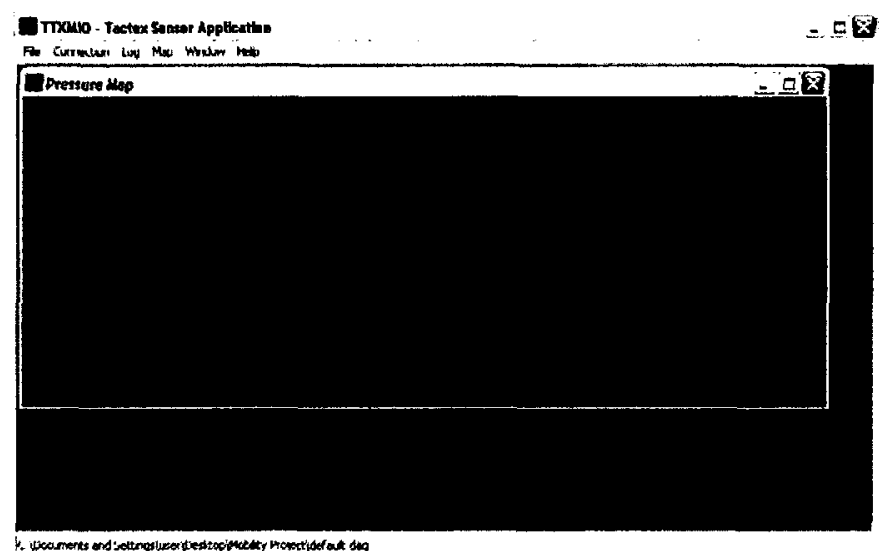

Figure B.5: Bed Mat Data Acquisition Software

7. Select Connection $\rightarrow$ Connect to 6 Sensors.

Now both the bed mats and the floor mat have established a connection with the computer and it is time to start acquiring data.

\section{A.5 Data Acquisition}

When acquiring data you must use both programs, one for the bed mats and one for the floor mat, simultaneously. After completing the steps below each patient should have two files associated with them, one with the floor mat data and one with the bed mat data.

1. From the Bed Mat window, select Log $\rightarrow$ Open Log File.

The Bed Mat software automatically creates a file for you to log the data. The file is saved to the "BED" folder on the desktop and is given a generic name with a time stamp. You must change this later in step 10.

2. Go back to Floor Mat window and select $\log \rightarrow$ Start recording.

Unlike the bed mat software the floor mat software gives you the choice of 
where you want to save the data file and what you want to name it. For convenience save all data to the same " $B E D$ " folder.

3. Name the floor mat $\log$ file floor_xxxx.csv $(x x x x=$ patient number $)$.

4. Click Open. Now both floor and bed data is being logged.

The patient should start the motion shown in Figure B.6 below. For healthy patients this motion will be repeated twenty times, but only one data file will be created. To distinguish between repetitions, it is important to follow the next steps.

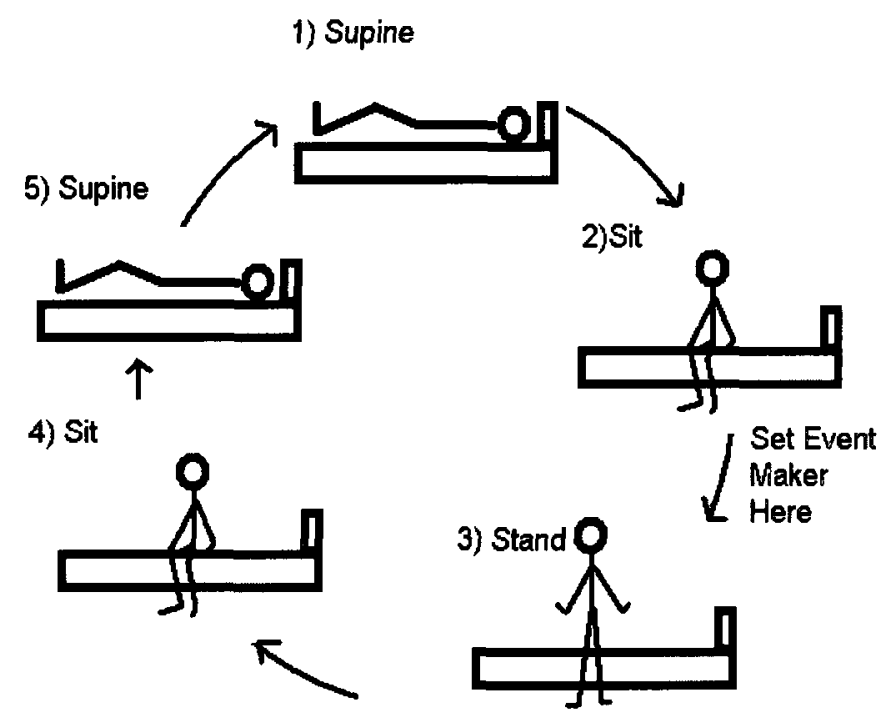

Figure B.6: Patient Motion

5. Once the patient has sat up from the supine position but before $s /$ he stands up, please mark an event. Do this by selecting Log $\rightarrow$ Set Event Marker from the Floor Mat window.

6. Go back to Bed Mat window and select $\log \rightarrow \log$ Event.

The Event number at the bottom of both the Data Acquisition windows should increment. This is shown in Figure B.7.

NOTE: Step 6 must be completed as quickly as possible after step 5. 


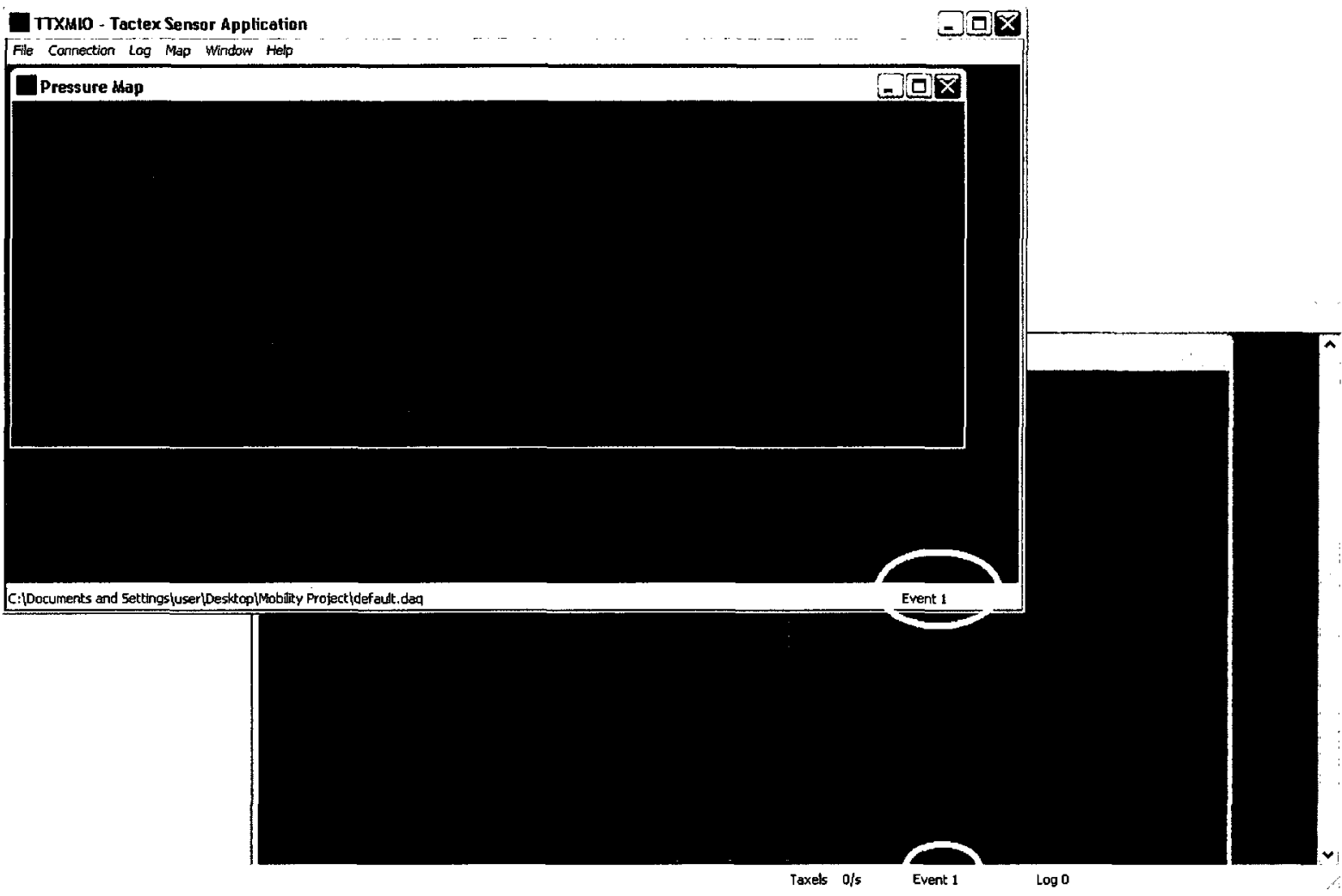

Figure B.7: Logging an Event

NOTE: As a shortcut, holding down "Ctrl+E" will also log an event.

7. Now that the event has been marked tell the sitting patient to stand.

8. Repeat steps 5-7 for the specified number of repetitions.

9. Once patient has completed all repetitions, in the Floor Mat window, select Log $\rightarrow$ Stop recording.

10. From the Bed Mat window, select Log $\rightarrow$ Close Log File.

11. Go to the "BED" folder and re-name the data_sensor_led_date_time.csv file bed $\mathrm{xxx} . \operatorname{csv}(\mathrm{xxxx}=$ patient number $)$.

NOTE: Do not close the Bed and Floor Mat Windows.

12. Repeat B.3 \#1-11 for each patient. 


\section{A.6 Troubleshooting}

In the event that the setup must be changed in anyway, or any of the equipment is powered down the user may run into some problems. This section outlines some common problems and explains what to do should they arise.

\section{In the "Searching" window, no NPort appears, ie. the list is empty.}

- Check the Ethernet connection from the NPort to the computer. This is the blue cable. Unplug the cable and plug it back in. The computer should recognize a "Local Area Connection"; if it does you should see the "Local Area Connection is O.K." icon in the bottom right hand corner of your screen as in Figure B.8 below. Otherwise the problem is the connection. Make sure the Ethernet cable is plugged in and restart your computer.

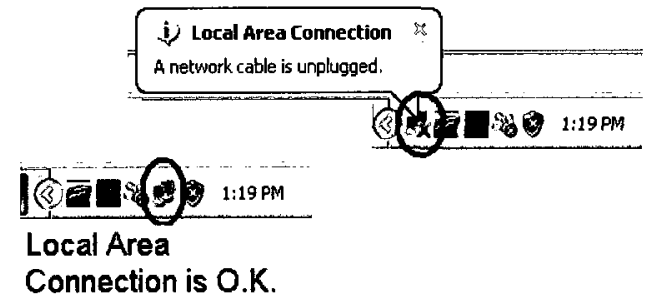

Figure B.8: Troubleshooting Local Area Connection

- If the Local Area Connection looks O.K. But you are still having the same problem open up the Network Connections from within the Control Panel. Click on the "Local Area Connection" and check the details in the bottom left hand corner of the Network Connections window. Make sure the IP Address is set to 192.168.127.250, as shown in Figure B.9 below.

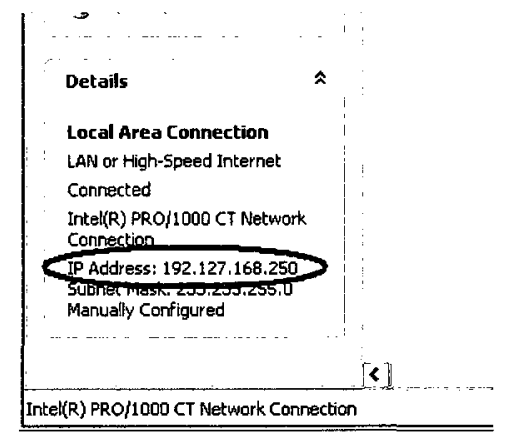

Figure B.9: Troubleshooting Local Area Connection

- Open up the cardboard box located under the bed, the large black box is the NPort, ensure the NPort is powered on.

- On the front panel of the NPort box the screen should list the IP address ensure that the IP address reads 192.168.127.254. If it doesn't the box was probably reset 
see problem $\# 2$.

\section{The NPort was accidentally "reset".}

- To reset the NPort you must:

a) Reset the IP Address to 192.168.127.254.

b) Ensure that COM ports 1-6 are set to "REAL Mode" and that COM ports 7 and 8 are disabled. To do this use the buttons located on the front panel of the NPort box.

\section{Some or all of the $\mathbf{4}$ floor tiles will not connect.}

- There is most likely a problem with the connection, physically disconnect the floor mat from the Data Acquisition Box and reconnect. Try reconnecting to the sensor tiles using the software.

4. The Tactex DAQ software is unable to establish a connection between the computer and the bed mats.

- Locate the default.daq file and the COM port mapping.ini file and ensure that

a) There are only $6 \mathrm{COM}$ ports listed in the COM port mapping file.

b) The port numbers in the COM port mapping file match the port numbers in the default file, as shown in Figure B.10 below.

c) Make sure the IP Address in the COM port mapping file is 192.168.127.254 as shown in Figure B.10 below.

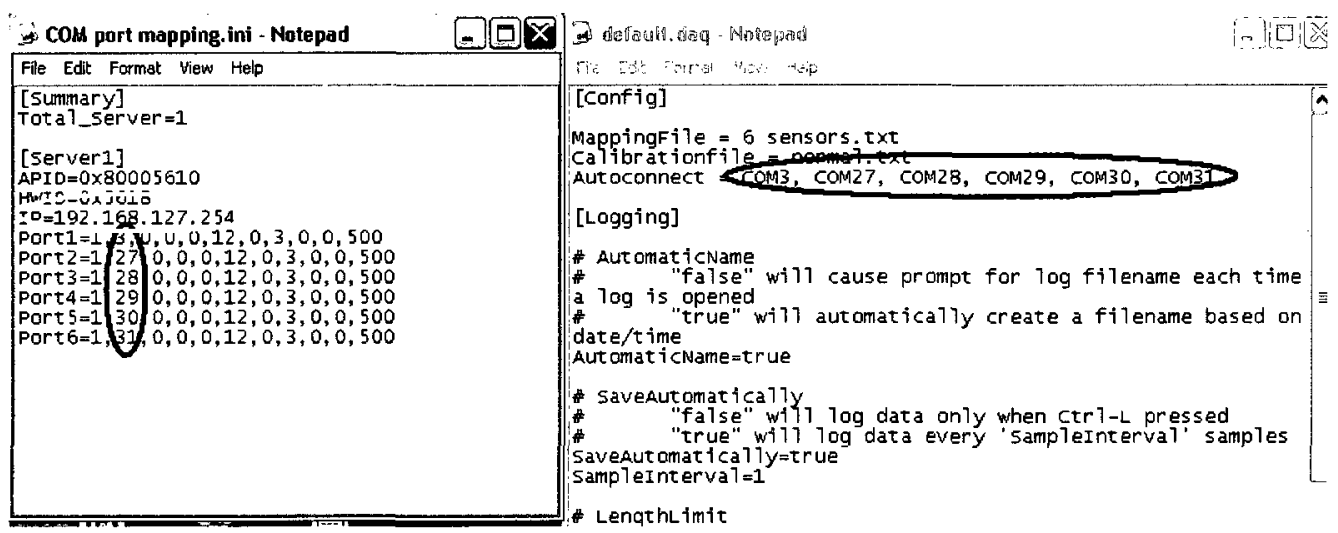

Figure B.10: Troubleshooting COM ports 